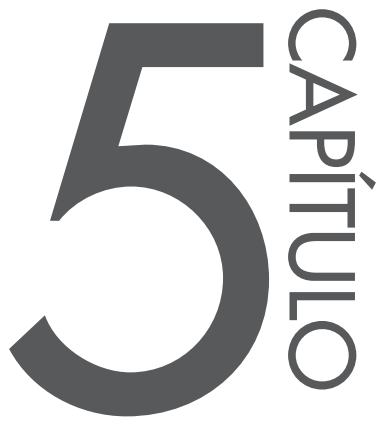

\title{
Desenvolvimento de uma Suspensão Automotiva do tipo Duplo A: Aplicação do módulo ADAMS/Car
}

A notar: Esta suspensão é comumente conhecida como "Double Wishbone", "Double A" ou ainda Duplo A. Ela é constituída por duas bandejas triangulares, as quais constituem elos entre o chassi do veículo e suas rodas. Classifica-se como uma suspensão independente e é reconhecida por ser mais robusta que outras suspensões como, por exemplo, a Macpherson. Isso pelo fato de possuir mais elementos mecânicos em sua formação.

Esse modelo apresenta uma fácil e precisa regulagem de seus parâmetros de geometria, como a variação da cambagem e a máxima variação de bitola. Devido a estes fatores este modelo de suspensão é mais comumente utilizado em eixos dianteiros de veículos de alto desempenho, por necessitarem de um ajuste mais fino dos parâmetros de geometria de suspensão. 


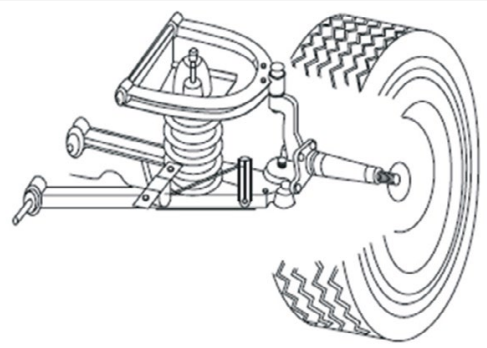

Ilustração de uma suspensão Duplo A.

Fonte: Valdeck, 2007.

Fonte: REIMPELL, J.; STOLL, H.; BETZLER, J. W. The automotive chassi: engineering principles, Butterworth-Heinemann, 2001.

Neste capítulo, será apresentado ao leitor o tutorial de construção de uma suspensão Duplo A, denominado_tutorial_duploa. A metodologia adotada para o desenvolvimento desse subsistema está dividida em 30 etapas, identificadas de acordo com as tarefas a serem executadas.

Até a Etapa 24, o leitor desenvolverá o modelo virtual, utilizando-se da interface Template Builder, conforme tratado no Capítulo 1. Nessas etapas, o leitor fará a parametrização do modelo, ou seja, criação de Hardpoint e Construction Frame, construção de partes (Parts), geometrias (Geometry), inserção de juntas e buchas (Attachments), criação de Communicators, entre outras ações pertinentes ao projetista nessa interface.

Nas etapas restantes, o leitor construirá um subsistema (Subsystem) associado ao Template da suspensão Duplo A e, em seguida, criará um Assembly entre o subsistema suspensão e um sistema de teste virtual do ADAMS/Car chamado Test Rig.

Ao final, após execução das etapas propostas, o leitor deverá obter o seguinte modelo virtual.

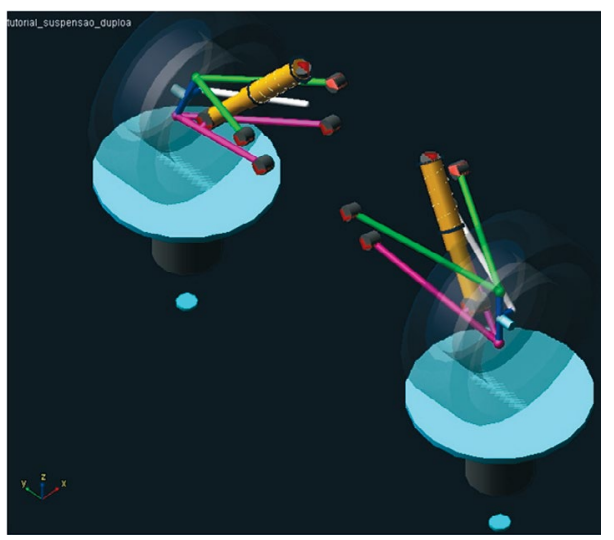

Obtenha o arquivo da suspensão

Duplo "A" no seguinte link: http://www.blucher.

com.br/materiaisdeapoio/mscadams/tutorial_

duploa.cdb.zip 


\section{Etapa 1 - Inicializando o Módulo ADAMS/Car}

Ao inicializar o módulo ADAMS/Car, as seguintes opções estarão disponíveis na janela de abertura: Standard Interface e Template Builder (item 1.2.1), conforme mostra a Figura 5.1. O usuário deverá marcar a opção Template Builder, uma vez que um novo projeto será criado, depois clicar na opção $O K$.

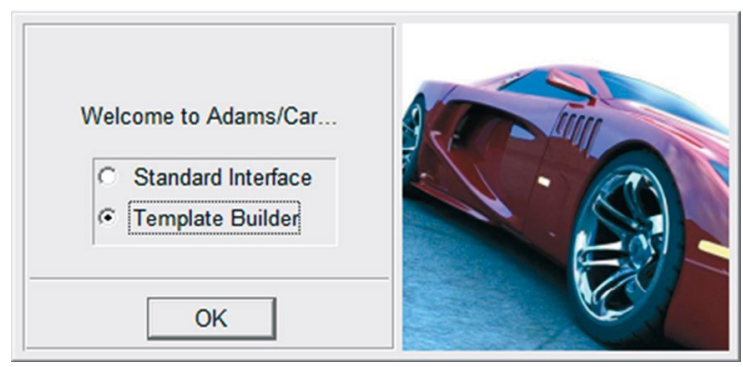

Figura 5.1 - Tela de inicialização do Módulo ADAMS/Car.

\section{Etapa 2 - Criando o Template}

- Abra o menu File e clique na opção New.

- Na caixa de diálogo aberta, digite o nome do projeto na caixa Template Name.

- Especifique o tipo de subsistema (Subsystem) a ser construído na opção Major Role, conforme ilustrado na Figura 5.2.

- $\quad$ Clique na opção $O k$.

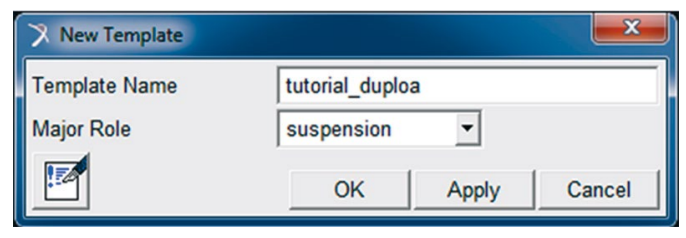

Figura 5.2 - Definição do New Template.

Etapa 3 - Definição do diretório de trabalho

- Abra o menu Tools e clique na opção Database Management $\rightarrow$ Create Database.

A Figura 5.3 ilustra a janela correspondente a essas ações. 


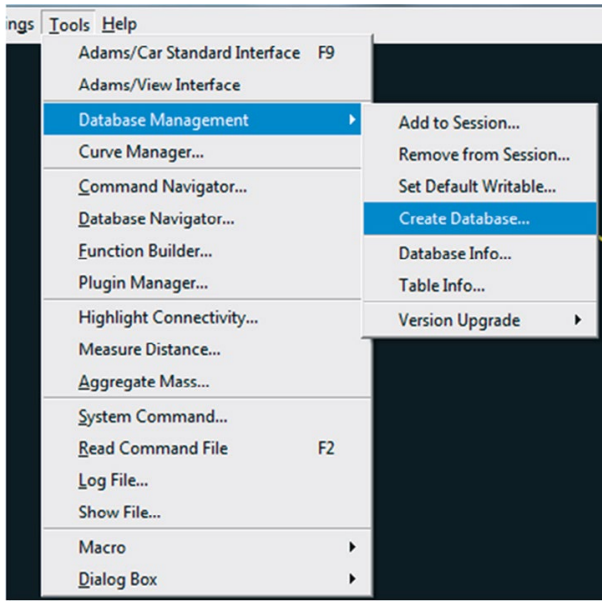

Figura 5.3 - Ilustração da janela referente à definição do diretório de trabalho.

- Na caixa de diálogo Create New Database preencha o campo Database Alias com o nome do diretório de trabalho e, no campo Database Path, informe seu caminho (Figura 5.4).

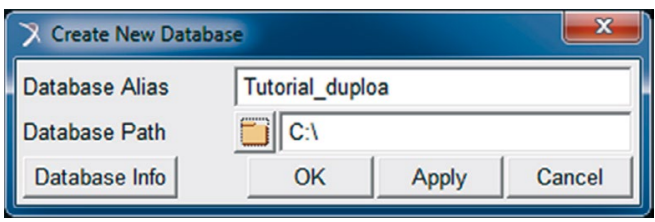

Figura 5.4 - Preenchimento sugerido da janela Create New Database.

- $\quad$ Clique em $O K$.

Uma nova janela será aberta para que o usuário aceite a criação do diretório de trabalho definido nas etapas anteriores, conforme ilustra a Figura 5.5.

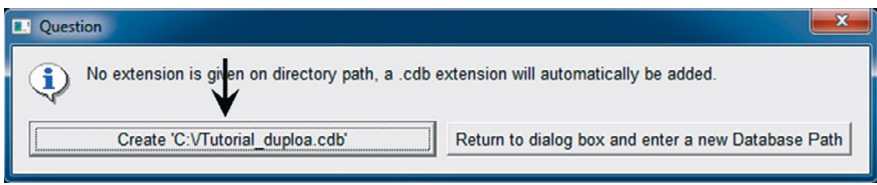

Figura 5.5 - Janela de confirmação da criação do diretório de trabalho.

- Uma mensagem de confirmação da criação do diretório será aberta, apenas para verificação por parte do usuário (Figura 5.6). Caso a tarefa tenha sido executada com sucesso, clique em Close. 


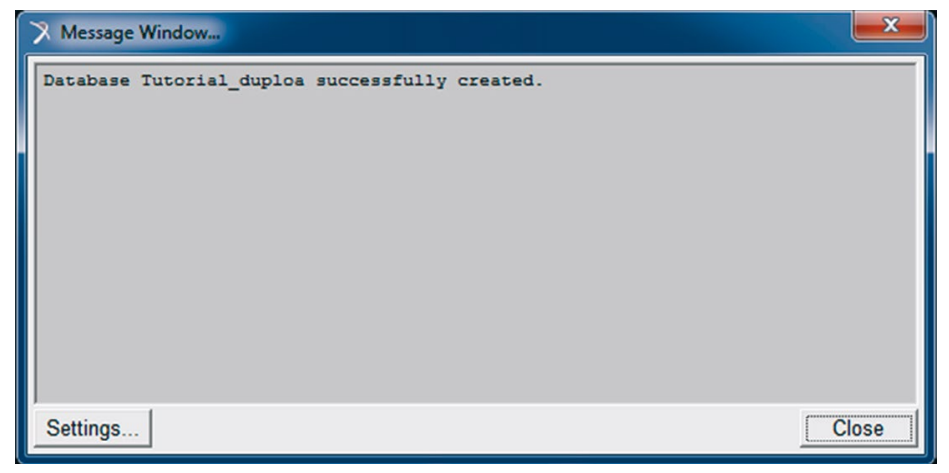

Figura 5.6 - Mensagem de alerta referente à criação do diretório de trabalho.

- Para obter informações sobre o diretório de trabalho criado, abra o menu Tools $\rightarrow$ Database Management $\rightarrow$ Database Info (Figura 5.7).

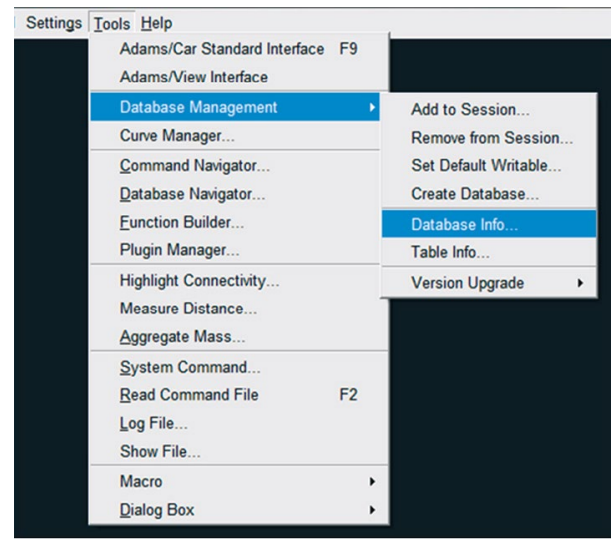

Figura 5.7 - Janela para obtenção de informações sobre o diretório de trabalho.

Em seguida, será exibida uma janela com a aparência da mostrada na Figura 5.8 


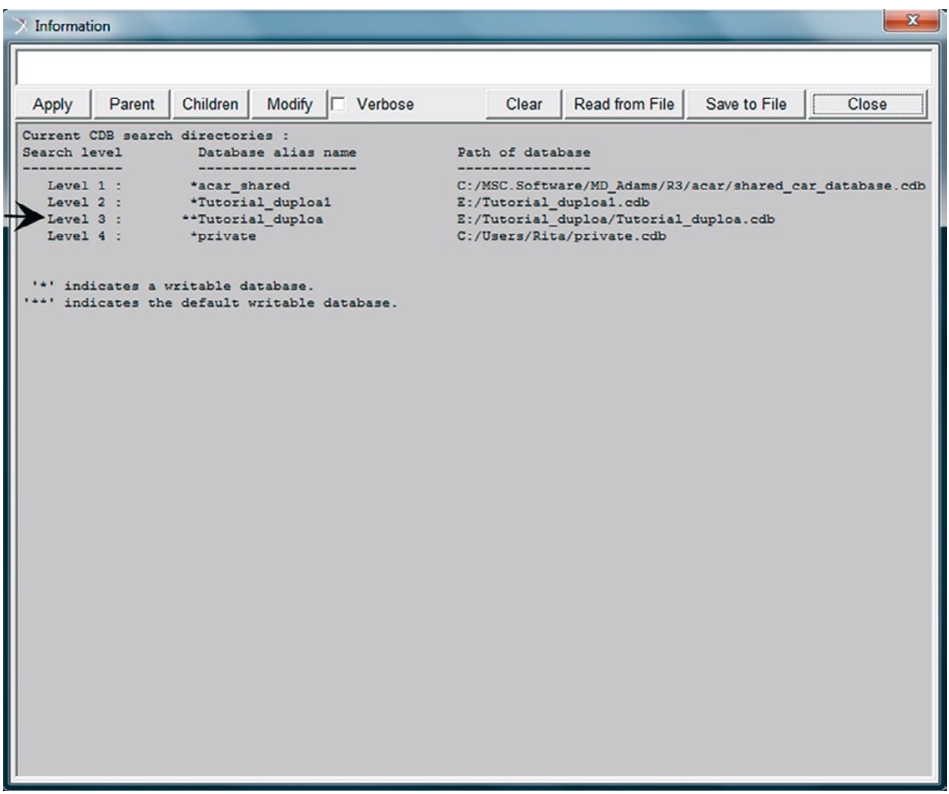

Figura 5.8 - Resultado da definição do diretório de trabalho.

A próxima etapa é definir o diretório de trabalho criado como sendo o diretório padrão, no qual os arquivos referentes ao projeto executado serão salvos. Para isso:

- Abra o menu Tools $\rightarrow$ Database Management $\rightarrow$ Set Default Writable (Figura 5.9).

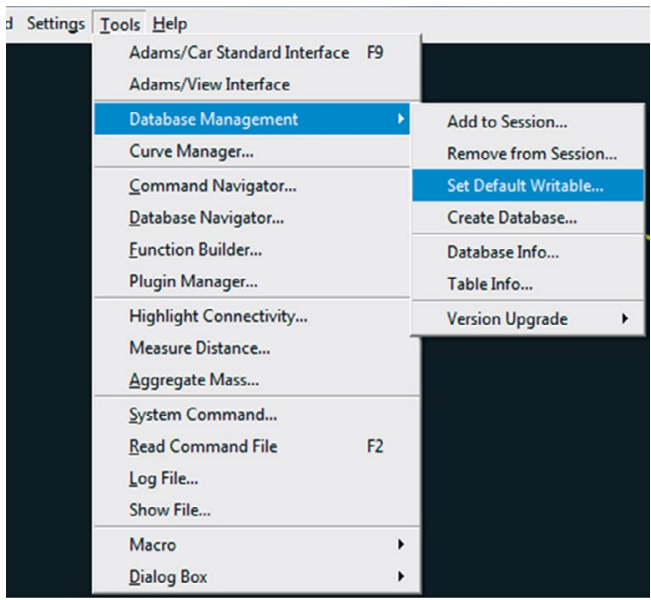

Figura 5.9 - Ilustração da opção a ser selecionada para definição do diretório padrão. 
- Será aberta uma caixa de diálogo (Set Default Writable Database), conforme ilustrado na Figura 5.10. No campo Database Name, selecione o diretório de trabalho criado anteriormente. Para tanto, clique sobre a seta à direita da aba.

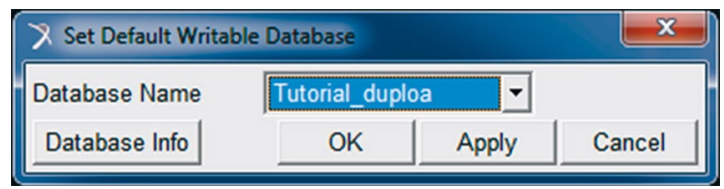

Figura 5.10 - Especificação do diretório de gravação de arquivos de projeto.

- $\quad$ Clique em $O K$.

\section{Etapa 4 - Parametrização da Geometria da Suspensão}

A notar: Para alterar/verificar unidades de medidas no ADAMS/Car, acesse o menu Settings $\rightarrow$ Units.

O primeiro passo para a construção de um subsistema suspensão é a criação dos Hardpoints. Dessa maneira, o modelo se torna parametrizado, facilitando, portanto, alterações geométricas, evitando, assim, o desenvolvimento de um novo projeto. Na Figura 5.11 é mostrado um esquema dos Hardpoints a serem definidos e sua respectiva representação em uma suspensão Double A. A numeração representada é coincidente com a da Tabela 5.1.

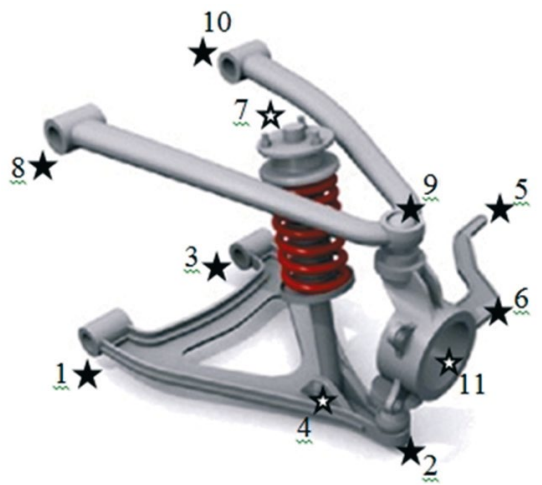

Figura 5.11 - Esquema dos Hardpoints a serem construídos.

Para criação dos Hardpoints ilustrados na Figura 5.12: 
- Acesse o menu Build, clique em Hardpoint e, em seguida, clique em New (Figura 5.12).

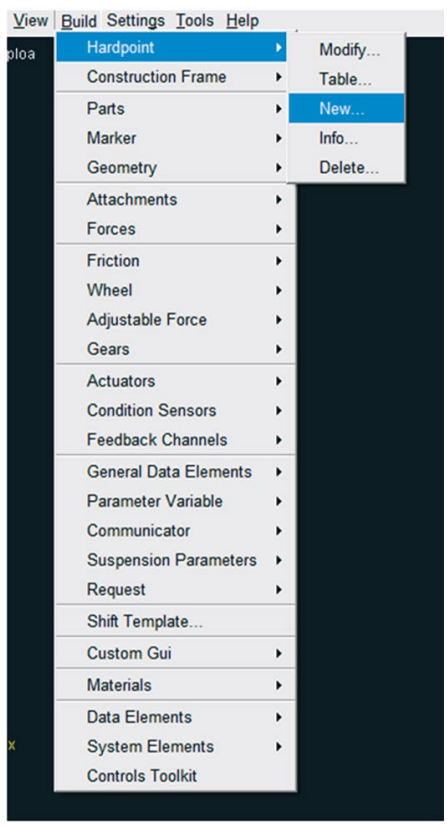

Figura 5.12 - Ativação da janela para criação de hardpoints.

Na caixa de diálogo Create Hardpoint defina o nome (Hardpoint Name) e selecione o tipo (Type). Se há simetria à esquerda - left -, à direita - right - ou caso não haja simetria defina como single.

A localização dos Hardpoints com relação às coordenadas globais $(x, y$ e $z)$ do sistema deverá ser inserida no campo Location (Figura 5.13). Caso haja algum erro nos campos preenchidos, esse erro será destacado na cor amarela, para alertar o usuário.

- Após preenchimento dos campos clique em Apply, para inserir os demais Hardpoints (Tabela 5.1) ou para finalizar, após inserção do último ponto clique em OK. A aba Type será selecionada para todos os Hardpoints criados como sendo right.

A notar: A simetria à direita ou à esquerda se refere ao fato de que os hardpoints criados se repetirão (serão espelhados) ou à direita ou à esquerda, relativamente ao referencial global do projeto. 


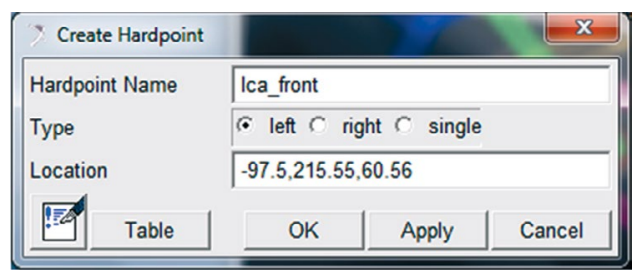

Figura 5.13 - Janela para criação de hardpoints.

A notar: Neste ponto, recomenda-se que se salve o projeto. Para tanto selecione a aba File $\rightarrow$ Save. Esta operação se repetirá em determinados pontos ao longo da construção da suspensão Duplo A.

Caso o usuário utilize a opção Save As, a tela mostrada a seguir aparecerá e se recomenda que ela seja preenchida conforme indicado.

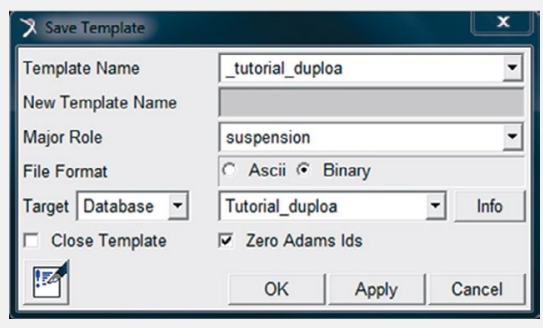

Figura ilustrativa da caixa de diálogo referente à gravação do Template.

\section{Tabela 5.1: Nomes e coordenadas dos hardpoints}

\begin{tabular}{c|c|c} 
& Nome & Coordenadas $(\mathbf{x}, \mathbf{y}, \mathbf{z})$ \\
\hline 1 & lca_front & $(-97.5,215.55,60.56)$ \\
\hline 2 & Ica_outer & $(-14.12,646.89,-106.22)$ \\
\hline 3 & Ica_rear & $(192.25,235.56,1.31)$ \\
\hline 4 & Iwr_strut_mount & $(8.43,529.97,-49.04)$ \\
\hline 5 & tierod_inner & $(150,290,76)$ \\
\hline 6 & tierod_outer & $(62.61,626.65,-43.76)$ \\
\hline 7 & top_mount & $(97.96,258.91,274.94)$ \\
\hline 8 & uca_front & $(-175.08,214.19,230.24)$ \\
\hline 9 & uca_outer & $(28.39,609.09,42)$ \\
\hline 10 & uca_rear & $(224.13,247.43,139.76)$ \\
\hline 11 & wheel_center & $(7.135,627.99,-32.11)$ \\
\hline
\end{tabular}


A notar: Repare que os Hardpoints com sigla de identificação "lca" (Lower Control Arm) se referem à bandeja inferior, já os com "uca" (Upper Control Arm) se referem à bandeja superior. Os Hardpoints identificados por "tierod" correspondem à união do sistema de direção ao subsistema suspensão. "lwr_strut_mount" e o "top_mount" são relativos à fixação do amortecedor na bandeja inferior e no chassi. Por fim, "wheel_center" localiza o centro da roda (Figura 5.11)

Após criar os onze Hardpoints, poderão ser feitas alterações em suas coordenadas, acessando-se o menu Build, clicando em Hardpoint e em seguida em Table. Uma tabela com o nome do Hardpoint e as respectivas localizações $x, y$ e $z$ abrirá na tela principal, possibilitando editá-la (Figura 5.14).

\begin{tabular}{|c|c|c|c|c|}
\hline \multicolumn{4}{|c|}{ X Hardpoint Modification Table } & \multirow[t]{2}{*}{$x$} \\
\hline & loc_x & loc_y & loc_z & \\
\hline hpl_Ica_front & -97.5 & 215.55 & 60.56 & \\
\hline hpl_lca_outer & -14.12 & 646.89 & -106.22 & \\
\hline hpl_Ica_rear & 192.25 & 235.56 & 1.31 & \\
\hline hpl_Iwr_strut_mount & 8.43 & 529.97 & -49.04 & \\
\hline hpl_tierod_inner & 150.0 & 290.0 & 76.0 & \\
\hline hpl tierod_outer & 62.61 & 626.65 & -43.76 & \\
\hline hpl_top_mount & 97.96 & 258.91 & 274.94 & \\
\hline hpl_uca_front & -175.08 & 214.19 & 230.24 & \\
\hline hpl_uca_outer & 28.39 & 609.09 & 42.0 & \\
\hline hpl_uca_rear & 224.13 & 247.43 & 139.76 & \\
\hline hpl_wheel_center & 7.135 & 627.99 & -32.11 & \\
\hline 1 & 1 & & & - \\
\hline Display: Single and & (- Left $C$ Right $C$ Both & Filter: * & Apply & Close \\
\hline
\end{tabular}

Figura 5.14 - Tabela de modificação da coordenada do hardpoint.

A notar: Perceba que na parte inferior desta janela há a opção Display. Nela ao selecionar as opções Left ou Right, haverá um ajuste automático dos sinais das coordenadas, de acordo com o sistema de coordenadas global. O termo hpl ou hpré inserido automaticamente no nome do Hardpoint pelo próprio software, de modo a indicar a que lado ele pertence, segundo o sistema de coordenadas global.

A Figura 5.15 ilustra a tela do software após criação dos Hardpoints. 


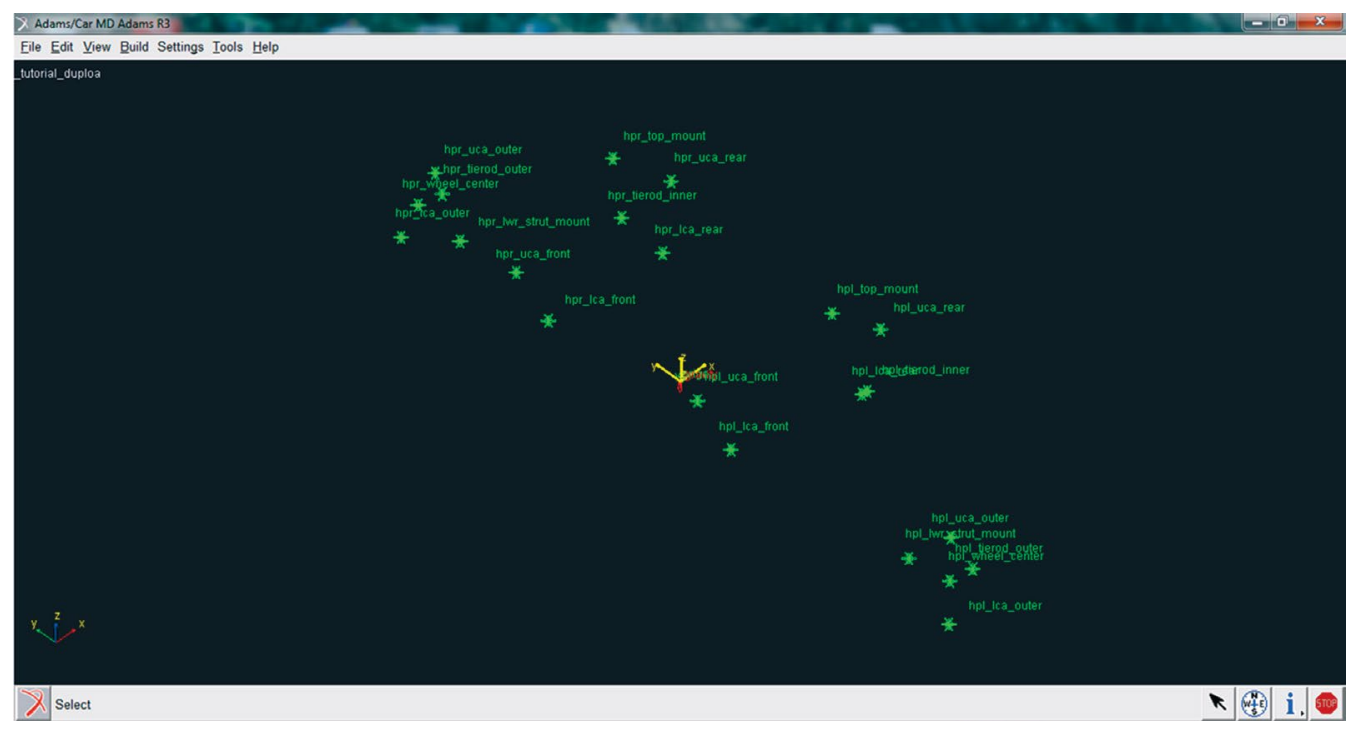

Figura 5.15 - Tela exibindo os hardpoints definidos.

Etapa 5 - Criação da bandeja inferior da suspensão (Lower Control Arm).

A bandeja inferior da suspensão é definida por três Hardpoints: lca_front; lca_rear e lca_outer, o que lhe confere uma forma triangular (Figura 5.11).

Para criação da bandeja, será necessário criar um General Part (item 1.2.5.2.1), o qual no ADAMS/Car é indicado pela abreviação gel (general part left) ou ger (general part right).

Para criação do General Part, é possível utilizar-se de dois tipos de construtores: New (criação do corpo rígido com características de geometria de massa e de material) ou Wizard (criação do corpo rígido baseado em duas geometrias possíveis Link/Arm, podendo-se definir a densidade do material, a partir da escolha do tipo ou como um input por parte do usuário).

- Para criação do General Part, acesse o menu Build $\rightarrow$ Parts $\rightarrow$ General Part $\rightarrow$ New (Figura 5.16). 


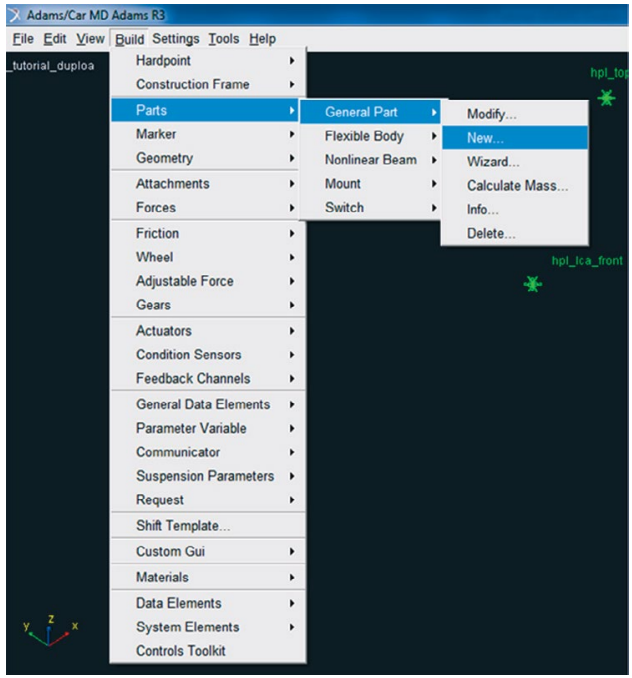

Figura 5.16 - Janela ilustrativa da opção selecionada para criação do General Part.

- $\quad$ Preencha a janela que se obtém ao acionar a opção, conforme mostrado na Figura 5.17.

O preenchimento dos campos: General Part, Coordinate Reference \#1, \#2, \#3 (Location Dependance), Coordinate Reference \#1 e \#2 (Orientation Dependance) pode ser realizado manualmente, ou se pode clicar com o botão direito do mouse no referido campo e optar por umas das opções disponíveis, sendo essa segunda forma de preenchimento, um atalho. Os campos que possuem a seta lateral se acionadas com um clique fornecem as opções disponíveis. A Figura 5.18 (a) e (b) ilustram o dito aqui, respectivamente.

Os dados relativos à geometria desse General Part, como massa, e momentos de inércia, inclusive o centro de massa, foram definidos, a priori, por um valor aleatório (como pode ser observado na Figura 5.17). Após inclusão da geometria (próxima etapa), esses dados serão recalculados. Caso o usuário já possua os valores corretos, estes podem ser inseridos sem a necessidade de recalculá-los posteriormente. 


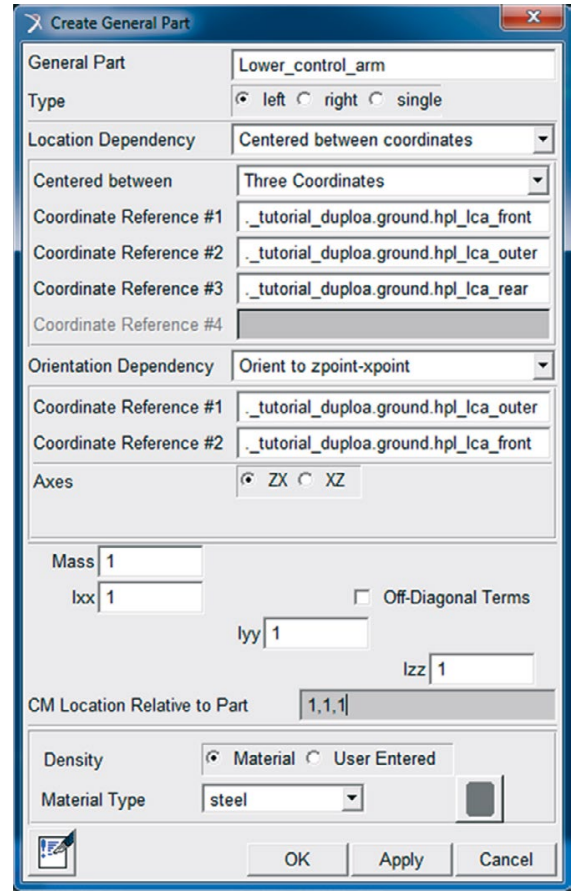

Figura 5.17 - Definição dos parâmetros de construção do General Part "Lower Control Arm".
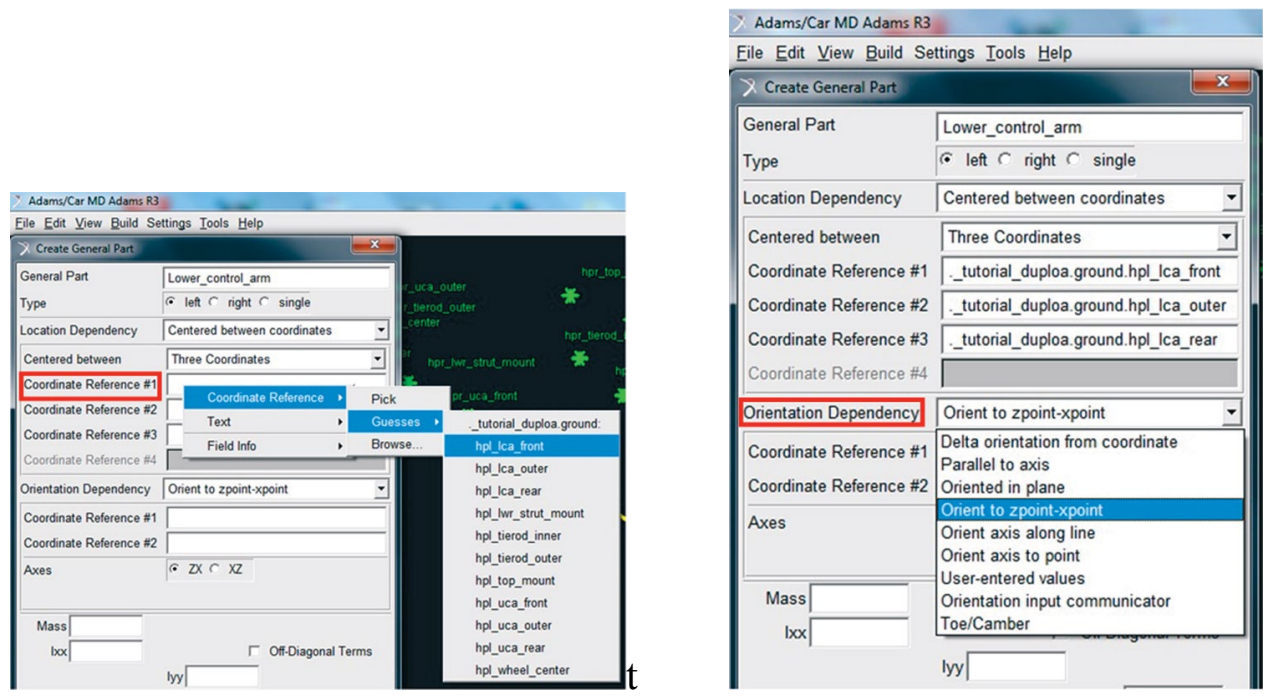

Figura 5.18 - Telas ilustrativas

(a) atalho para seleção de coordenadas, por exemplo, Coordinate Reference \#1;

(b) acionamento de seta lateral para definição de opção (Orientation Dependency). 
A notar: Verifique que no campo Orientation Dependency foi selecionada a opção Orient to zpoint-xpoint. Ao selecioná-la juntamente com a opção ZX no campo Axes (Figura 5.18 (a), o usuário define que o eixo “ $Z$ ” do General Part criado (Lower_ control_arm) será paralelo ao vetor formado entre a entidade (General Part) e a coordenada \#1 (Coordinate Reference \#1). Veja que a origem do vetor está no General Part e sua extremidade no Hardpoint hpl_lca_outer.

- Após preenchimento dos campos da Figura 5.17, clique em $O K$.

A Figura 5.19 ilustra a tela principal do software, destacando a criação do General Part.

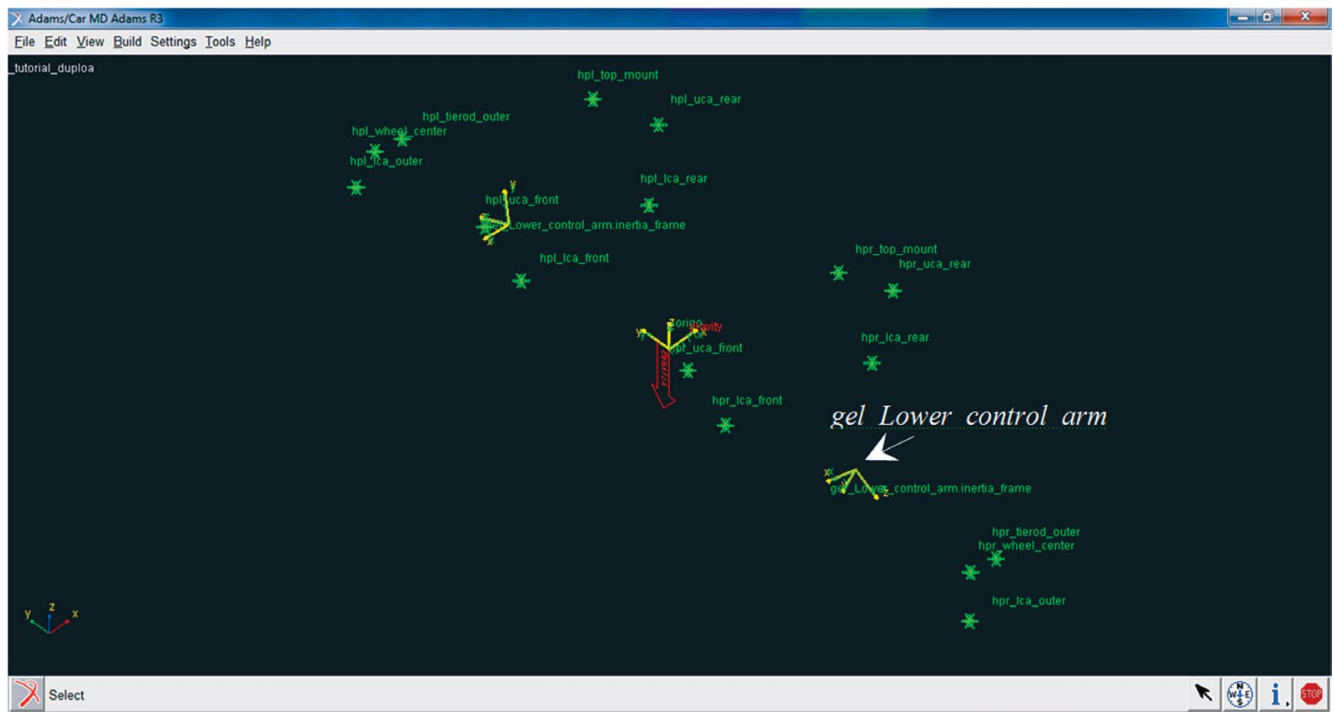

Figura 5.19 - General Part "Lower Control Arm” construído e sua orientação.

\section{Etapa 6 - Criação da geometria relacionada ao General Part "Lower Control Arm"}

A bandeja inferior da suspensão Lower Control Arm, a qual está relacionada ao General Part criado na etapa anterior, é composta por duas geometrias cilíndricas do tipo Link e três esféricas, que serão criadas pela ferramenta Ellipsoid, localizadas nas extremidades dos Links.

A notar: Para definir a geometria do tipo Link são necessários dois Hardpoints localizados em ambas as extremidades do Link, além da definição de um raio. 
- $\quad$ Para construção da primeira geometria Link, o qual será formado pelos Hardpoints hpl_lca_front e hpl_lca_outer, acesse o menu Build $\rightarrow$ Geometry $\rightarrow$ Link $\rightarrow$ New, como mostrado na Figura 5.20.

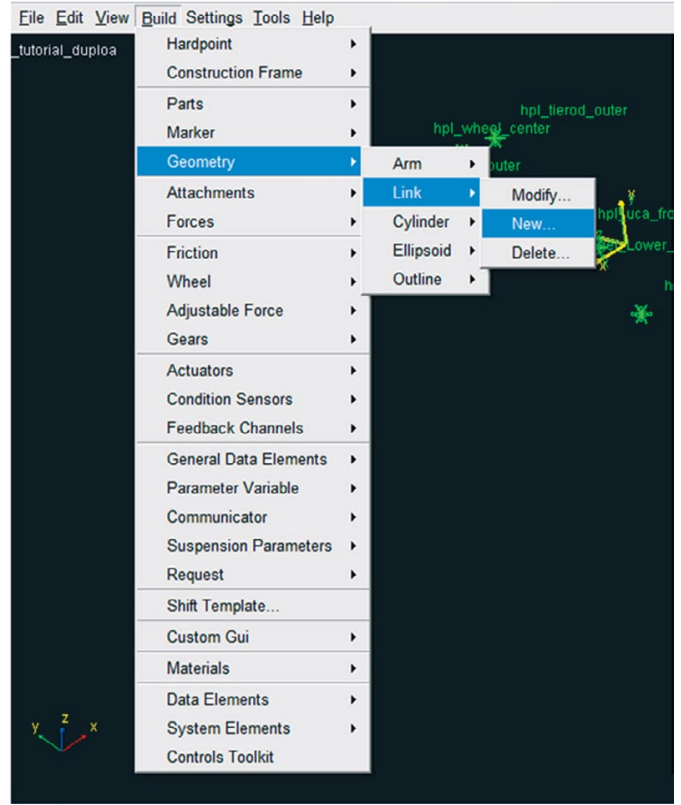

Figura 5.20 - Janela referente à construção da geometria Link.

- A janela Create Link Geometry será aberta e deverá ser preenchida conforme ilustrado na Figura 5.21. Observe que a opção para calcular as propriedades de massa do General Part (Calculate Mass properties of General Part) deve ser assinalada. Isso no caso de se desejar atualizar os dados de propriedades de massa inseridos na Etapa 5.

- Ao finalizar, clique na opção $O K$.

Repare que o preenchimento das abas General Part, Coordinate Reference \#1 e Coordinate Reference \#2 pode ser manual ou pelo acionamento do botão direito do mouse sob a aba, conforme ilustrado na Figura 5.18 (a). Nesse caso, recomenda-se escolher Coordinate Reference $\rightarrow$ Guesses, onde se abre uma janela elencando todos os Hardpoints, $h p l$ e $h p r$ disponíveis no projeto. 


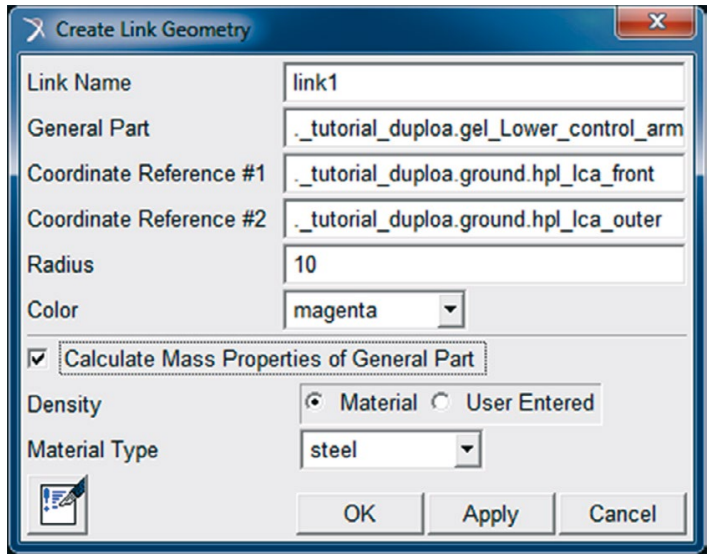

Figura 5.21 - Criação do Link associado ao General Part criado na Etapa 5.

O Link construído está ilustrado na Figura 5.22.

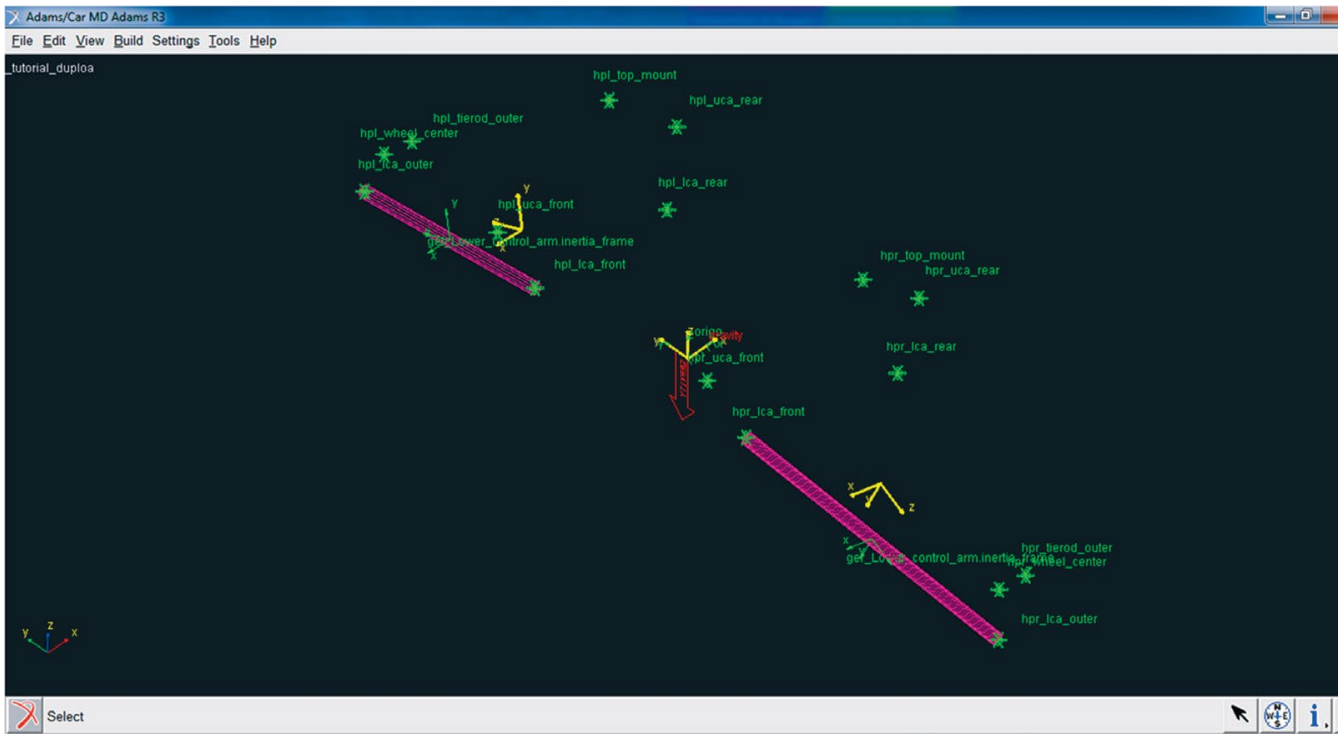

Figura 5.22 - Link 1 construído.

- Repita o mesmo procedimento descrito anteriormente para construção do Link 2, o qual terá em suas extremidades os Hardpoints hpl_lca_rear e hpl_lca_outer. A Figura 5.23 ilustra como os campos da caixa de diálogo devem ser preenchidos.

- $\quad$ Clique em $O K$.

- $\quad$ Salve seu projeto. 
A notar: Dependendo da disponibilidade do usuário, o presente projeto pode ser realizado de uma só vez, ou interrompido para que depois se dê continuidade a ele. No entanto, quando o Template é aberto a partir de um arquivo salvo, este pode aparecer na tela principal sem todos os detalhes construtivos, normalmente vistos pelo usuário, tais como juntas, especificação de Hardpoints, Construction Frame, General Part etc. Para que todas essas entidades passem a ser visiveis novamente no modelo, posicione o cursor em qualquer ponto da tela de construção (menos em pontos do modelo) e clique com o botão direito do mouse. Na janela que se abrirá, selecione a opção Toogle Icon Visibility <v>.

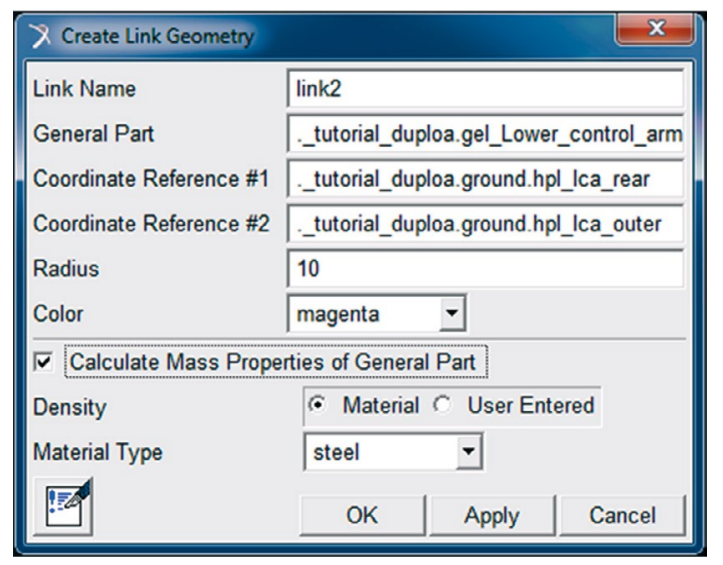

Figura 5.23 - Criação do Link 2 associado ao General Part criado na Etapa 5.

Nas extremidades dos Links 1 e 2 serão construídos volumes esféricos, a partir da ferramenta Ellipsoid. Para tanto, acesse o menu Build $\rightarrow$ Geometry $\rightarrow$ Ellipsoid $\rightarrow$ New (Figura 5.24).

A notar: A geometria Ellipsoid pode ser utilizada para gerar geometrias esféricas, desde que os raios definidos em cada coordenada (x, y e z) sejam iguais. 


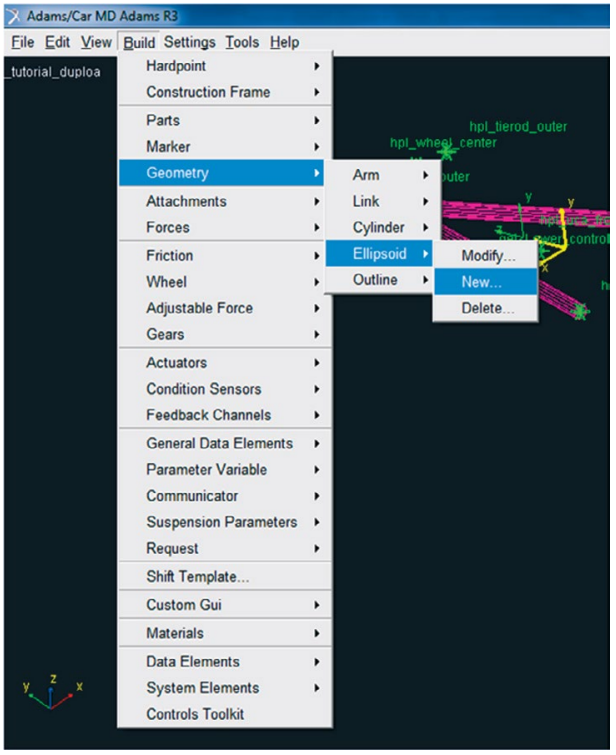

Figura 5.24 - Janela para criação da geometria esférica nas extremidades do Link.

- A janela Create Ellipsoid Geometry será aberta. Os campos deverão ser preenchidos conforme ilustrado na Figura 5.25. Observe que essa primeira esfera terá como referência o Hardpoint hpl_lca_front, e pertencerá ao General Part gel_Lower_control_arm. Lembre-se que o campo Calculate Mass Properties of General Part deverá estar selecionado para atualização das propriedades de massa do General Part Lower_control_arm.

- $\quad$ Clique em $O K$.

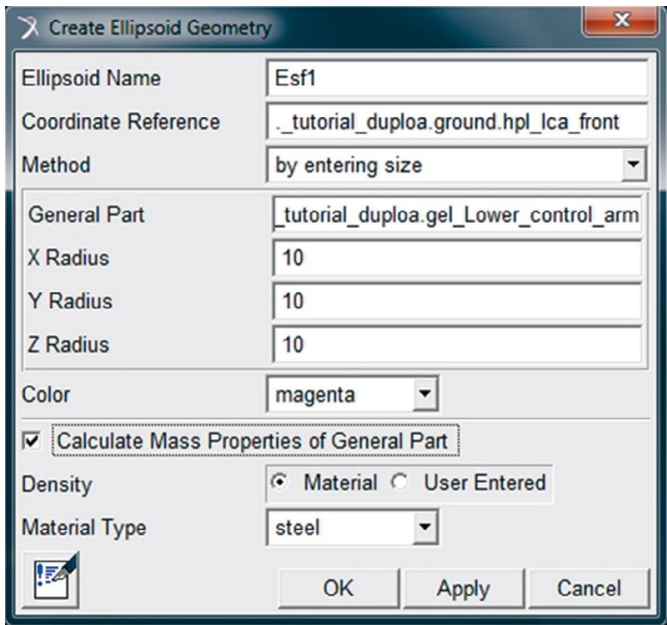

Figura 5.25 - Preenchimento da caixa de diálogo para criação da primeira esfera. 
- O procedimento do item anterior deverá ser repetido para construção do segundo volume esférico. A segunda esfera será localizada no Hardpoint hpl_lca_rear. O preenchimento da janela Create Ellipsoid Geometry para essa geometria está ilustrado na Figura 5.26.

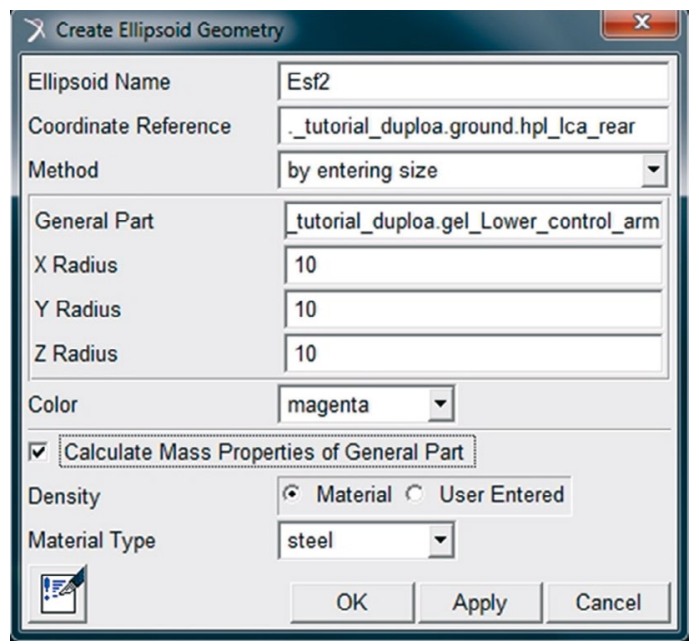

Figura 5.26 - Preenchimento da caixa de diálogo para criação da segunda esfera.

- A terceira esfera será construída na intersecção dos Links 1 e 2. A Figura 5.27 ilustra o preenchimento da janela Create Ellipsoid Geometry para construção dessa geometria.

- $\quad$ Clique em $O K$.

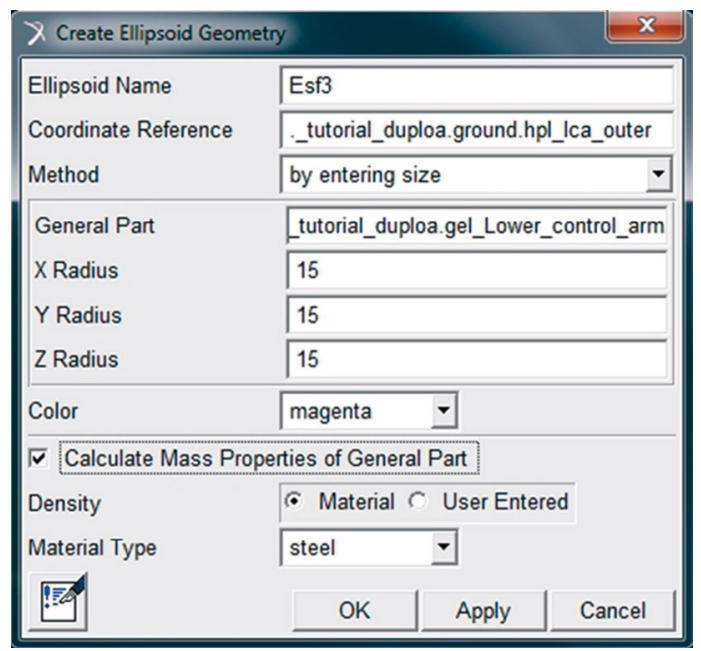

Figura 5.27 - Preenchimento da caixa de diálogo para criação da terceira esfera. 
Após construção das geometrias que compõem a bandeja da suspensão, o General Part Lower_control_arm será atualizado em relação às suas propriedades de massa.

- Aproxime a imagem de um dos lados da suspensão, pressionando o botão direito do mouse em qualquer ponto da tela, menos em pontos da geometria do modelo virtual, selecione a opção Zoom In/Out e mova o cursor para o efeito desejado.

- Em seguida, na tela principal do software, clique com o botão direito do mouse sobre o General: Part gel_Lower_control_arm. Em seguida, clique em Modify (Figura 5.28).

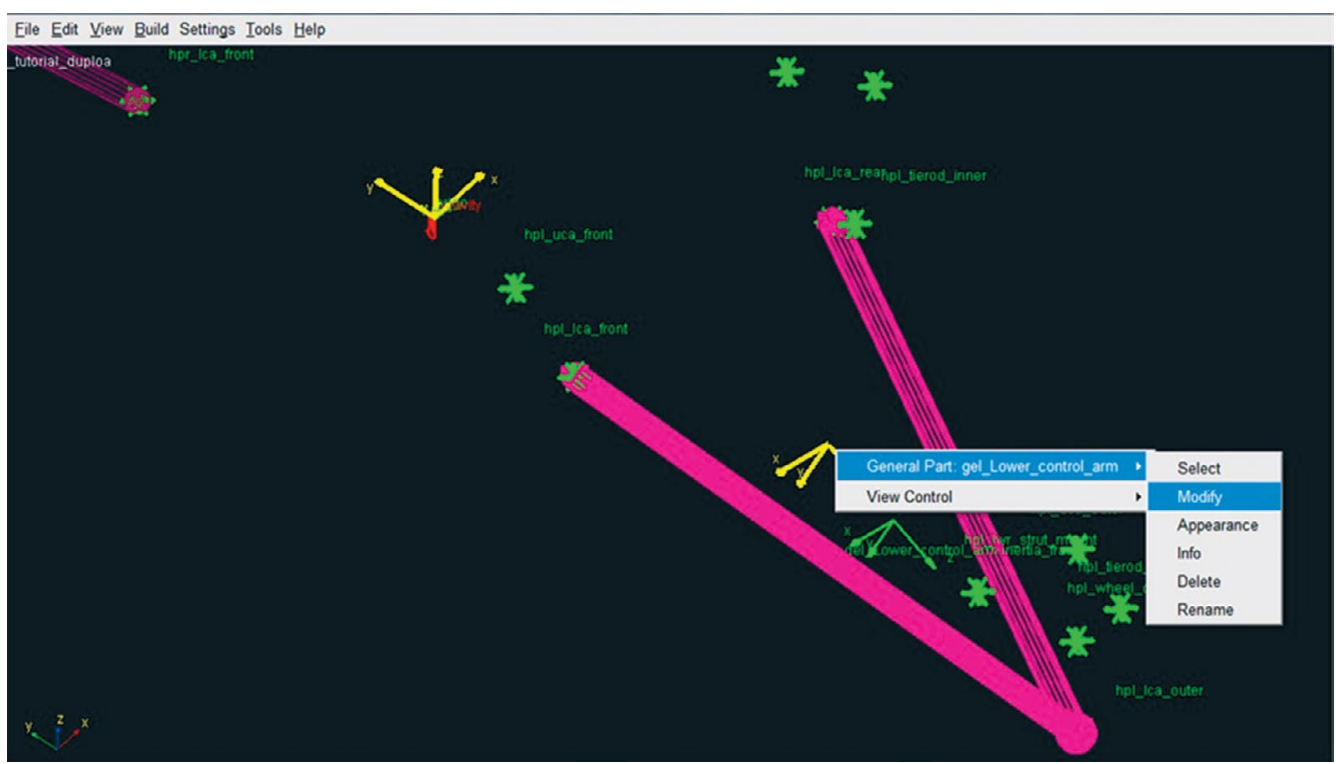

Figura 5.28 - Opção para atualização das propriedades do General Part Lower_control_arm a partir da opção Modify.

- A janela Modify General Part será aberta. Para atualização das propriedades, clique no ícone referente à calculadora (canto inferior direito), como destacado na Figura 5.29. Após essa ação, os dados serão automaticamente recalculados.

- $\quad$ Clique em $O K$. 


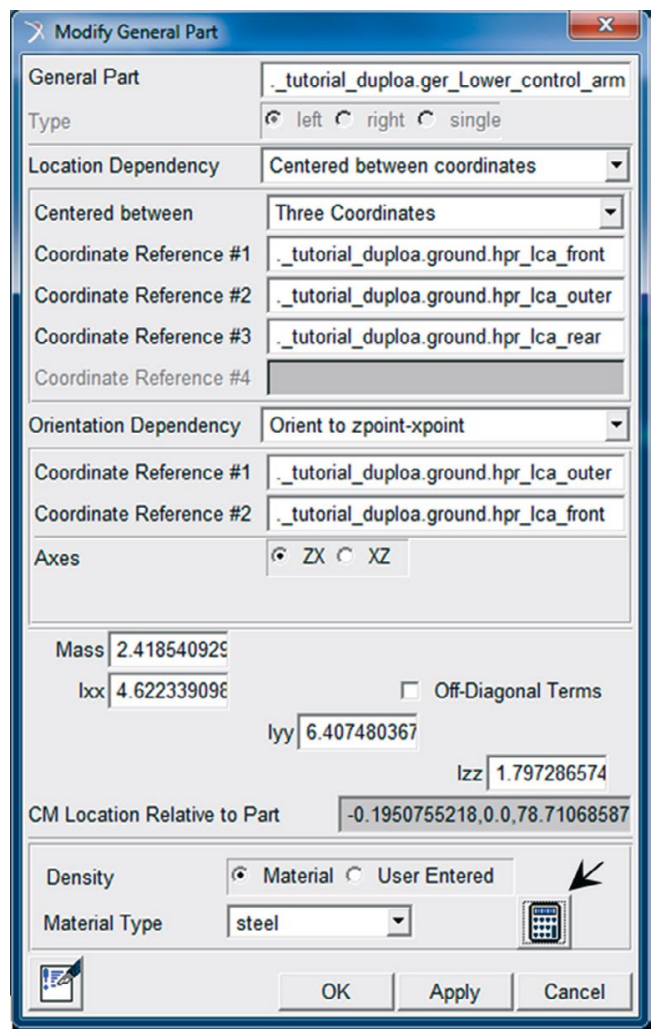

Figura 5.29 - Atualização das propriedades do General Part "gel_Lower_control_arm".

- Salve seu projeto.

\section{Eatapa 7 - Construção da fixação inferior do amortecedor na bandeja Inferior}

Nesta etapa, será construída a geometria para ancoragem à bandeja.

Para tanto, é necessário que sejam criados Construction Frames para orientação dos Links. Para isto:

- Acesse o menu Build Construction Frame $\rightarrow$ New (Figura 5.30). 


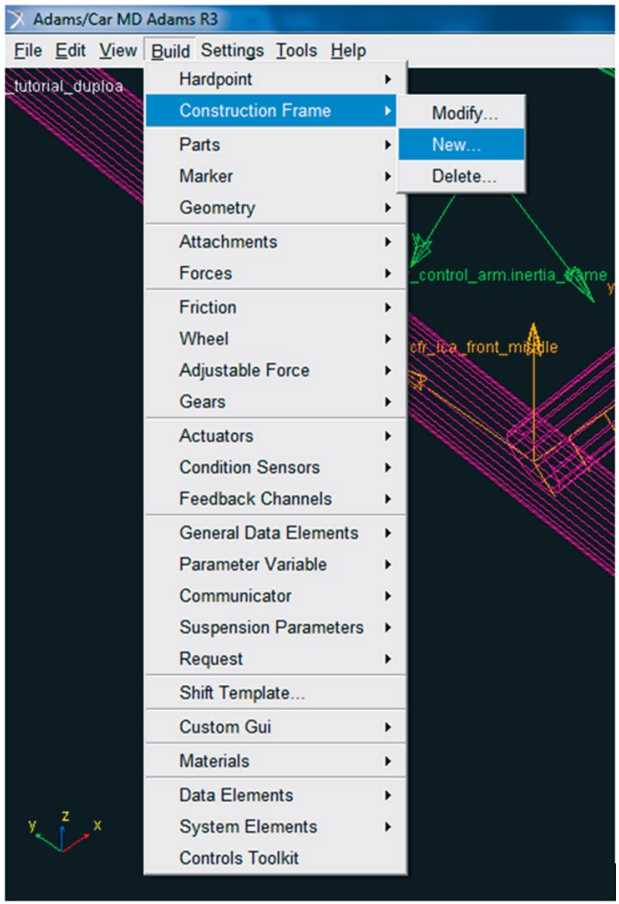

Figura 5.30 - Janela para criação do Construction Frame para fixação do amortecedor.

A notar: Trata-se de um elemento do ADAMS/Car, que é utilizado sempre que uma determinada entidade necessita para sua criação, não apenas da definição de coordenadas para localização como o Hardpoint, mas também de uma orientação (Orientation Dependancy). Como ele é um elemento parametrizável, ele acompanha as alterações realizadas nos Hardpoints relacionados a ele.

Além disto, perceba que na construção de determinados elementos do modelo, onde houver a necessidade de se definir coordenadas (Coordinate Reference \#1, \#2, \#3 ou \#4 ou ainda, I ou J Coordinate Reference) essas abas serão sempre preenchidas ou por Hardpoint ou Construction Frame.

- A janela Create Construction Frame será aberta e os campos deverão ser preenchidos conforme ilustrado na Figura 5.31. Note que este Construction Frame será construído entre os seguintes Hardpoints: $h p l \_l c a$ front e hpl_lca_outer.

- $\quad$ Clique em $O K$.

Repare que a janela Location Dependency (assinalada na Figura 5.31), se acionada na seta lateral direita, fornece seis opções de localização. Selecione Located on a line, o que solicita ao usuário a entrada de duas coordenadas de referência (Coordinate Reference \#1 e Coordinate Reference \#2). 
A notar: A opção Relative Location (\%) fornece ao usuário a possibilidade de definir a localização do Construction Frame, com base na distância entre as coordenadas (x, $\mathrm{y}, \mathrm{z})$ de \#1 e \#2.

No caso, considerando-se as coordenadas dos Hardpoint hpl_lca_front $e$ hpl_lca_ outer; a distância entre eles é dada pela diferença de coordenadas entre hpl_lca_outer $e$ hpl_lca_front, que multiplicada por $72 \%$ e somada aos valores de coordenadas de hpl_lca_front, fornece o posionamento do Construction Frame.

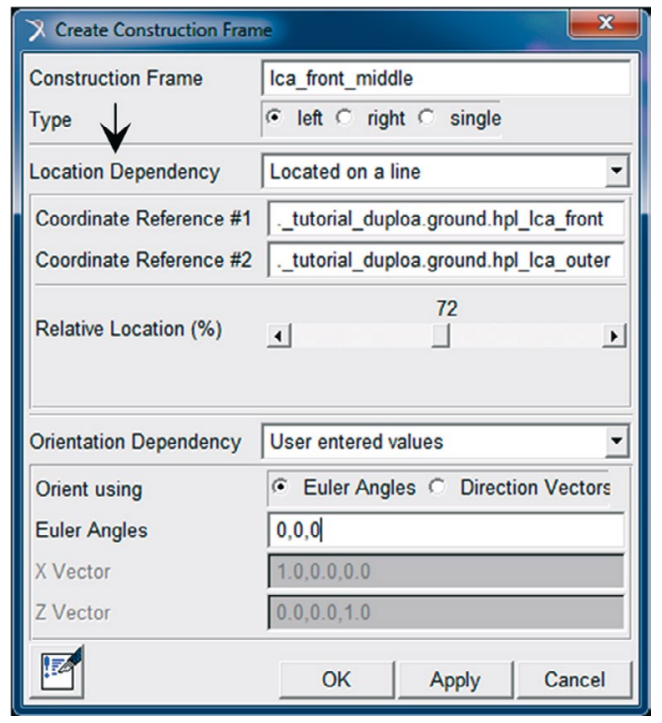

Figura 5.31 - Janela de construção do Construction Frame lca_front_middle.

- A Figura 5.32 destaca o Construction Frame criado, indicado pela seta branca. 


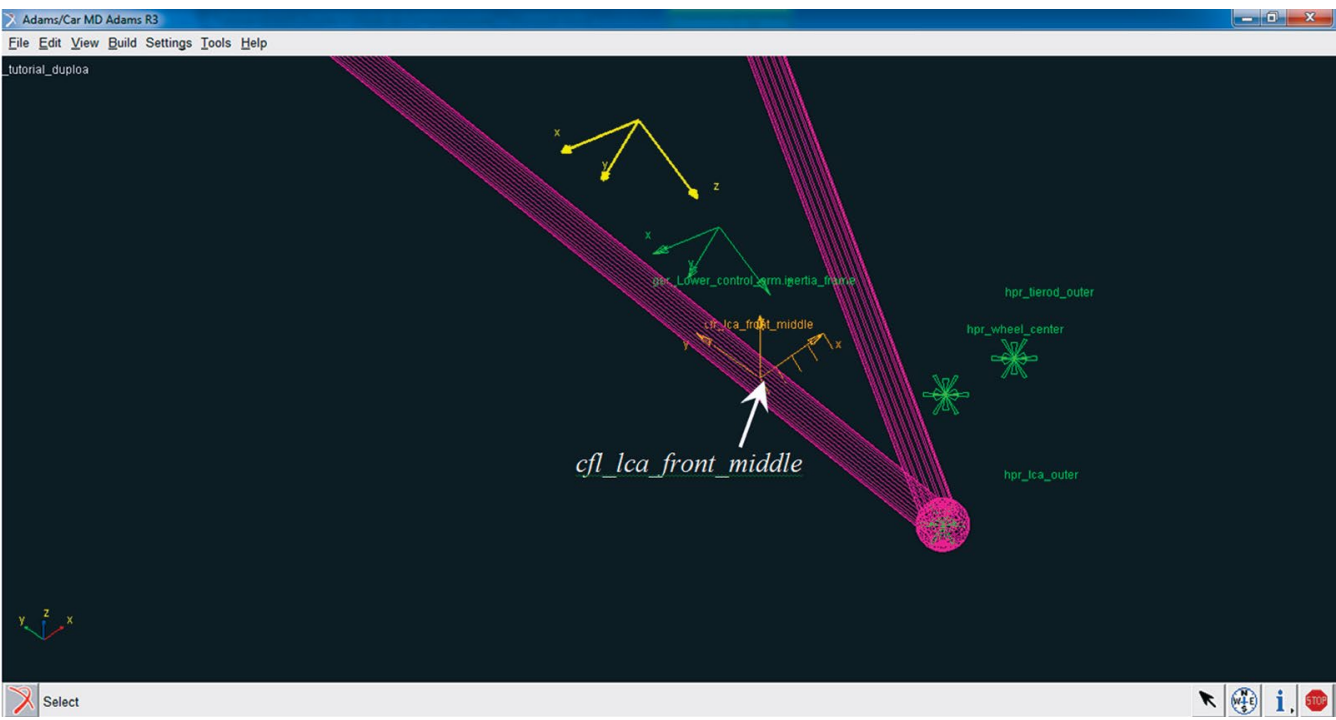

Figura 5.32 - Detalhe do primeiro Construction Frame criado na bandeja inferior da suspensão.

- Em seguida, será criado um novo Construction Frame, no qual as informações a serem fornecidas devem seguir o que mostra a Figura 5.33. Após a construção, repare que esses Construction Frame possuem seus eixos paralelos.

\begin{tabular}{|c|c|c|}
\hline \multicolumn{2}{|l|}{ X Create Construction Frame } & $x$ \\
\hline \multirow{2}{*}{$\begin{array}{l}\text { Construction Frame } \\
\text { Type }\end{array}$} & \multicolumn{2}{|l|}{ Ica_rear_middle } \\
\hline & (- left $C$ right $C$ sing & \\
\hline Location Dependency & Located on a line & $\checkmark$ \\
\hline Coordinate Reference \#1 & _tutorial_duploa.ground & ca_rear \\
\hline Coordinate Reference \#2 & ._tutorial_duploa.ground & ca_outer \\
\hline Relative Location (\%) & $\stackrel{72}{\perp}$ & $\perp$ \\
\hline Orientation Dependency & User entered values & $\nabla$ \\
\hline Orient using & (- Euler Angles $\subset$ Dir & Vectors \\
\hline Euler Angles & $0,0,0 \mid$ & \\
\hline$X$ Vector & $1.0,0.0,0.0$ & \\
\hline Z Vector & $0.0,0.0,1.0$ & \\
\hline EEd & Apply & Cancel \\
\hline
\end{tabular}

Figura 5.33 - Janela de construção do Construction Frame lca_rear_middle. 
A Figura 5.34 destaca o Construction Frame criado, indicado pela seta branca.

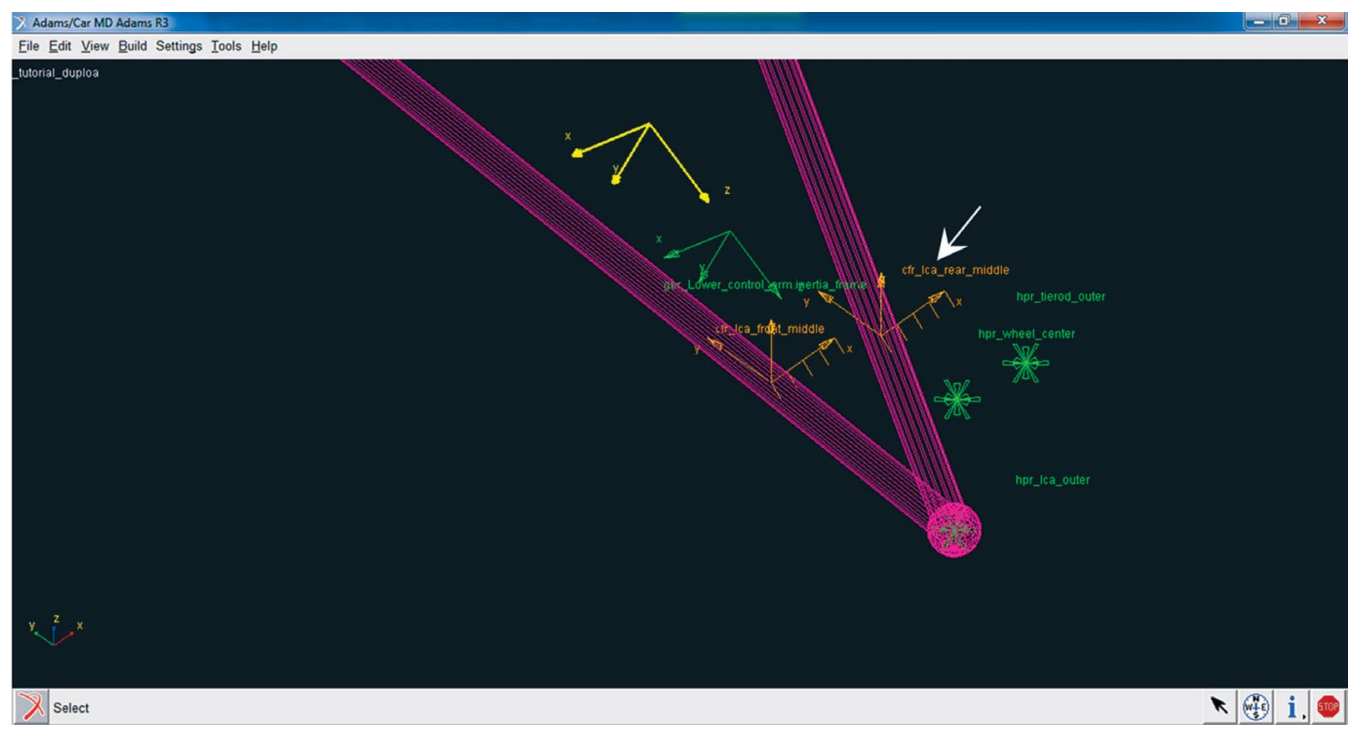

Figura 5.34 - Detalhe do segundo Construction Frame criado na bandeja inferior da suspensão.

Com a construção dos Construction Frame, é possível criar, a partir dessas entidades, um Link que ligue os dois braços da bandeja, onde será fixado o amortecedor.

- $\quad$ Para a criação deste Link, acesse o menu Build $\rightarrow$ Geometry $\rightarrow$ New (Figura 5.35). 


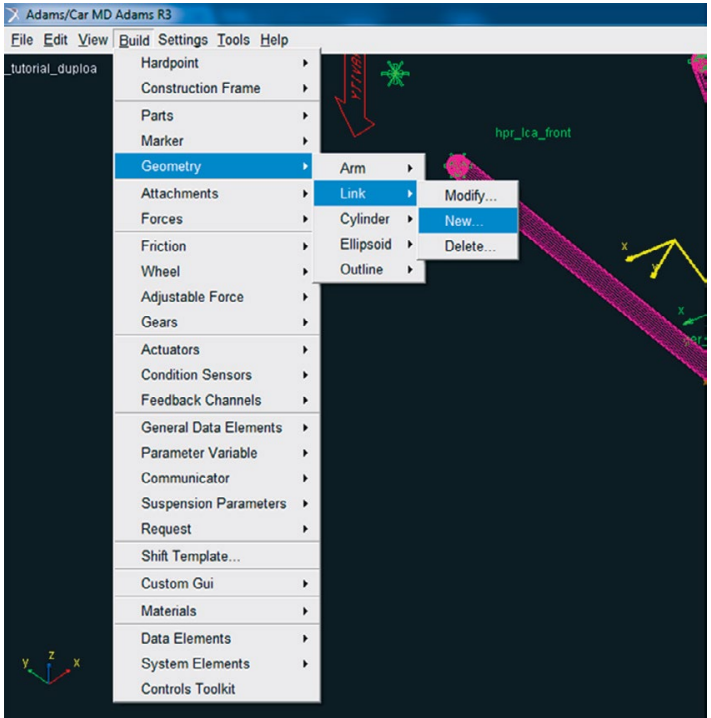

Figura 5.35 - Criação do Link para fixação do amortecedor na bandeja inferior da suspensão.

A criação do Link três deve atender às especificações mostradas na Figura 5.36 .

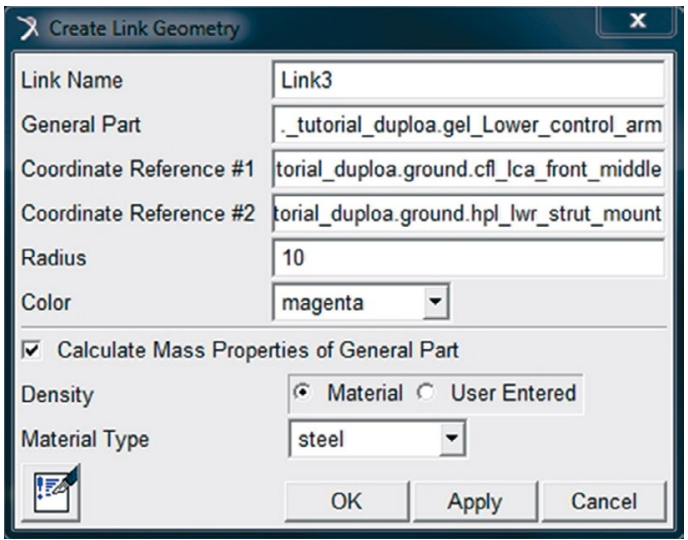

Figura 5.36 - Construção do Link de união entre os braços de suspensão da bandeja inferior - Link 3.

- $\quad$ Clique em Apply e preencha as especificações para o Link 4, como mostrado na Figura 5.37. 


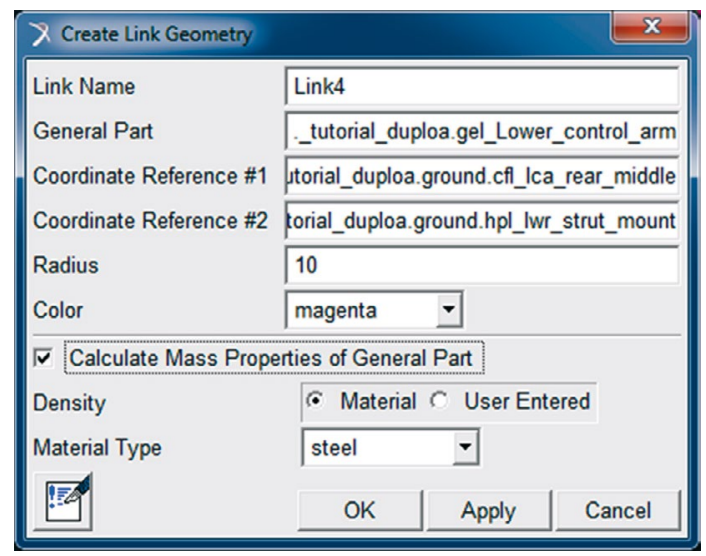

Figura 5.37 - Construção do Link de união entre os braços de suspensão da bandeja inferior - Link 4.

- $\quad$ Em seguida, clique em $O K$, e verifique se os Links foram criados corretamente, conforme ilustrado na Figura 5.38.

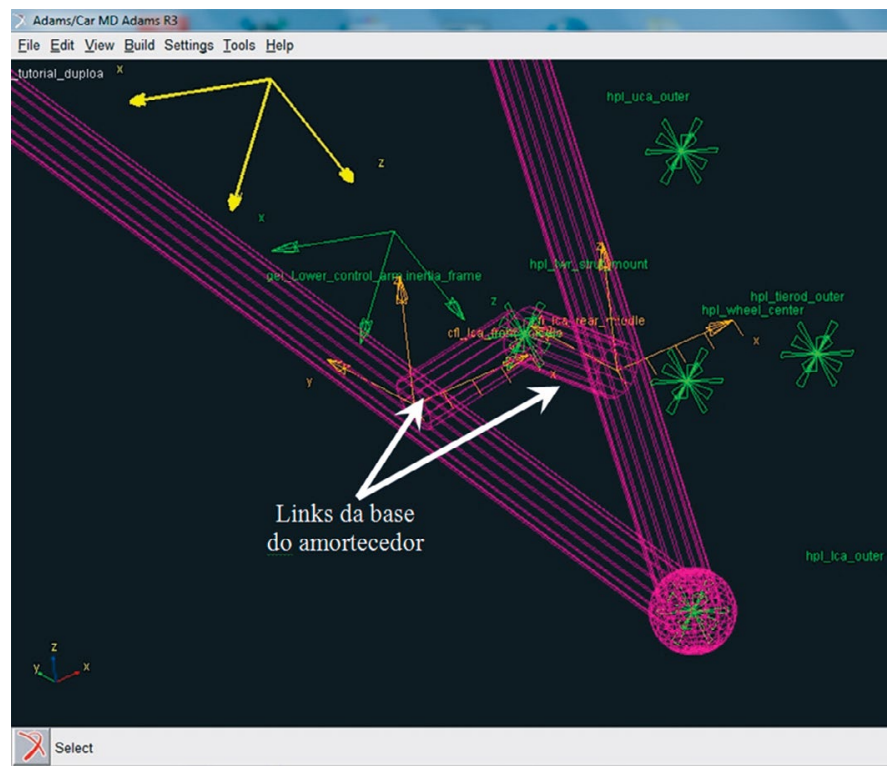

Figura 5.38 - Links 3 e 4, construídos para fixação do amortecedor.

- $\quad$ Salve seu projeto.

Etapa 8 - Criação da bandeja superior da suspensão (Upper Control Arm)

A bandeja superior da suspensão é definida por três Hardpoints: uca_front; uca_rear e uca_outer, sendo, portanto, triangular. 
Para criação da bandeja superior, da mesma forma que para a bandeja inferior, será necessário criar um General Part.

A construção do General Part é realizada acessando-se o menu Parts $\rightarrow$ General Part New (Figura 5.39)

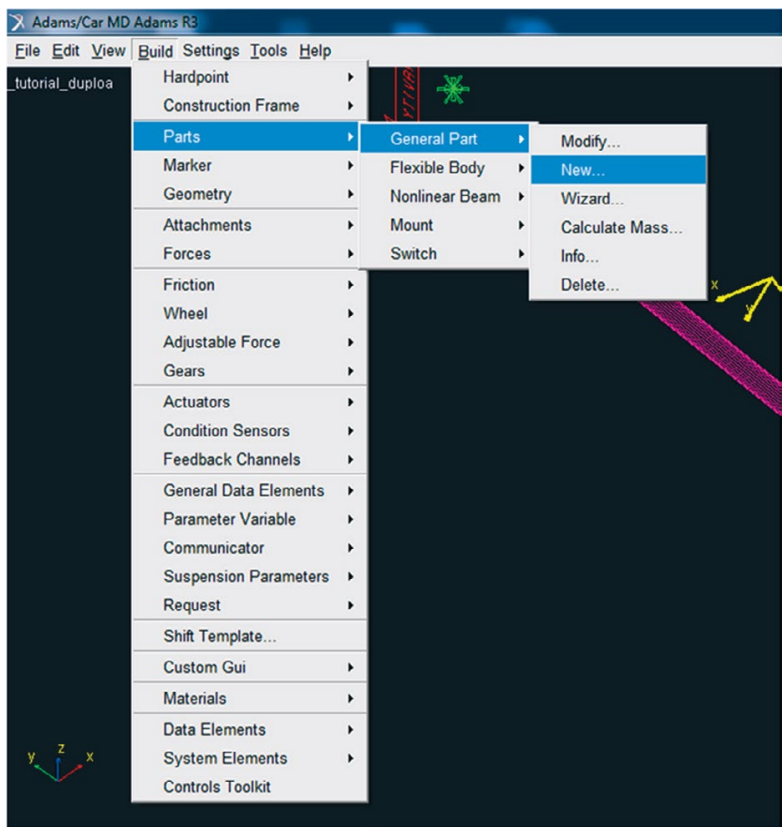

Figura 5.39 - Criação do General Part referente à bandeja superior.

O preenchimento da janela de criação do General Part deve ser como ilustra a Figura 5.40. 


\begin{tabular}{|c|c|}
\hline \multicolumn{2}{|l|}{ Create General Part } \\
\hline \multirow{2}{*}{$\begin{array}{l}\text { General Part } \\
\text { Type }\end{array}$} & Upper_control_arm \\
\hline & (- left $C$ right $C$ single \\
\hline Location Dependency & Centered between coordinates \\
\hline \multirow{5}{*}{$\begin{array}{l}\text { Centered between } \\
\text { Coordinate Reference \#1 } \\
\text { Coordinate Reference \#2 } \\
\text { Coordinate Reference \#3 } \\
\text { Coordinate Reference \#4 }\end{array}$} & Three Coordinates \\
\hline & _tutorial_duploa1.ground.hpl_uca_outer \\
\hline & ._tutorial_duploa1.ground.hpl_uca_front \\
\hline & ._tutorial_duploa1.ground.hpl_uca_rear \\
\hline & \\
\hline Orientation Dependency & Orient to zpoint-xpoint \\
\hline \multirow{2}{*}{$\begin{array}{l}\text { Coordinate Reference \#1 } \\
\text { Coordinate Reference \#2 }\end{array}$} & ._tutorial_duploa1.ground.hpl_uca_outer \\
\hline & _tutorial_duploa1.ground.hpl_uca_front \\
\hline \multicolumn{2}{|r|}{ (. $Z X \subset X Z$} \\
\hline \multicolumn{2}{|l|}{ Mass 1} \\
\hline \multirow[t]{3}{*}{$|x x| 1$} & $\Gamma$ Off-Diagonal Terms \\
\hline & |yy 1 \\
\hline & $|z z| 1$ \\
\hline \multicolumn{2}{|c|}{ CM Location Relative to Part $\quad 1,1,1$} \\
\hline \multirow{2}{*}{$\begin{array}{l}\text { Density } \\
\text { Material Type }\end{array}$} & Material $\subset$ User Entered \\
\hline & teel \\
\hline EDd & Cancel \\
\hline
\end{tabular}

Figura 5.40 - Janela de criação do General Part Upper_control_arm.

- Ao finalizar o preenchimento, clique em $O K$.

A Figura 5.41 destaca o General Part criado, indicado pela seta branca.

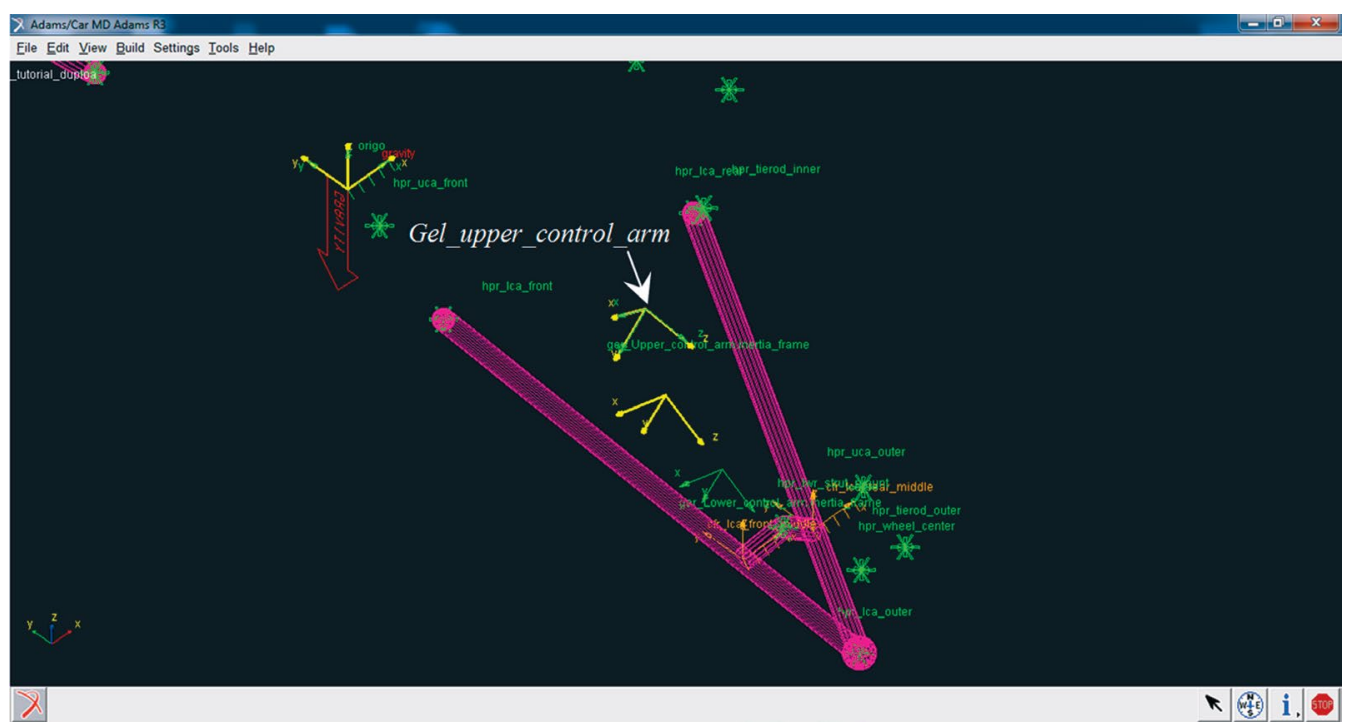

Figura 5.41 - General Part referente à criação do Upper_Control_Arm. 


\section{Etapa 9 - Criação da geometria relacionada ao General Part "Upper Control Arm"}

Após criação do General Part, parte-se para a definição da geometria da bandeja inferior, a partir da criação de geometrias denominadas Link. Para tanto:

- Acesse o menu Build $\rightarrow$ Geometry $\rightarrow$ Link $\rightarrow$ New (Figura 5.42).

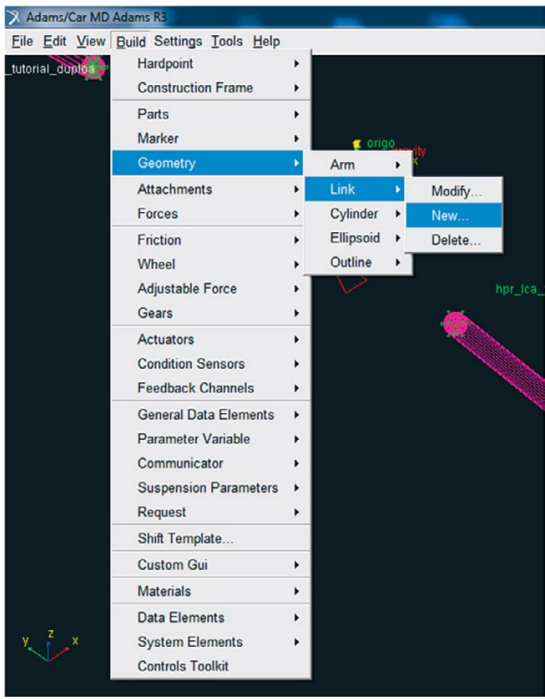

Figura 5.42 - Janela ilustrando a opção a ser ativada para criação de Links na bandeja superior (Upper Control Arm).

- Preencha a janela Create Link Geometry, de acordo com o especificado na Figura 5.43.

- $\quad$ Clique em Apply.

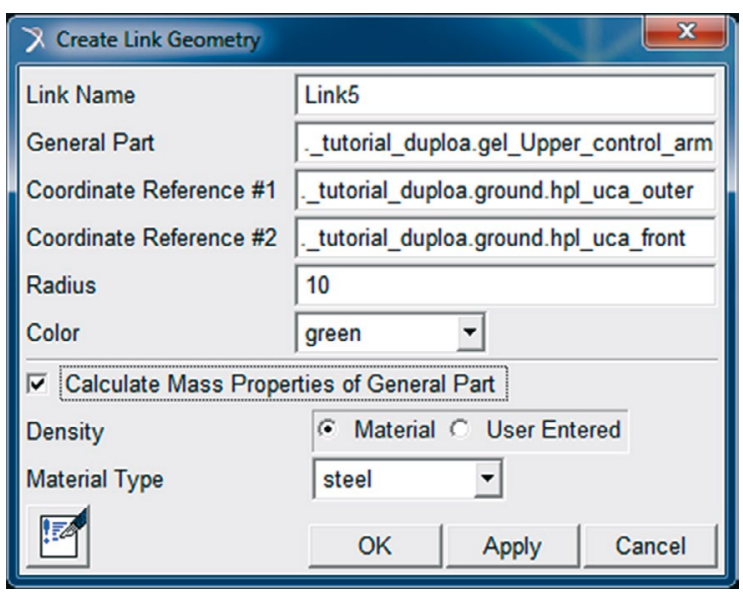

Figura 5.43 - Criação do Link 5, associado ao General Part criado na Etapa 8. 
- Repita o procedimento para o Link 6, preencha a janela Create Link Geometry de acordo com o especificado na Figura 5.44.

- $\quad$ Clique em $O K$.

\begin{tabular}{|c|c|c|}
\hline \multicolumn{3}{|l|}{ C Create Link Geometry } \\
\hline Link Name & \multicolumn{2}{|l|}{ Link6 } \\
\hline General Part & \multicolumn{2}{|c|}{ |_tutorial_duploa.gel_Upper_control_arm } \\
\hline Coordinate Reference \#1 & \multicolumn{2}{|c|}{-_tutorial_duploa.ground.hpl_uca_outer } \\
\hline Coordinate Reference \#2 & \multicolumn{2}{|c|}{-_tutorial_duploa.ground.hpl_uca_rear } \\
\hline Radius & \multicolumn{2}{|l|}{10} \\
\hline Color & green & \\
\hline \multicolumn{3}{|c|}{$\sqrt{\checkmark}$ Calculate Mass Properties of General Part } \\
\hline Density & \multicolumn{2}{|c|}{ (- Material C User Entered } \\
\hline Material Type & \multicolumn{2}{|l|}{ steel } \\
\hline 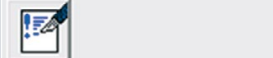 & Apply & Cancel \\
\hline
\end{tabular}

Figura 5.44 - Criação do Link 6, associado ao General Part criado na Etapa 8.

A Figura 5.45 destaca as geometrias Links criadas e associadas ao General Part "gel_Upper_control_arm".

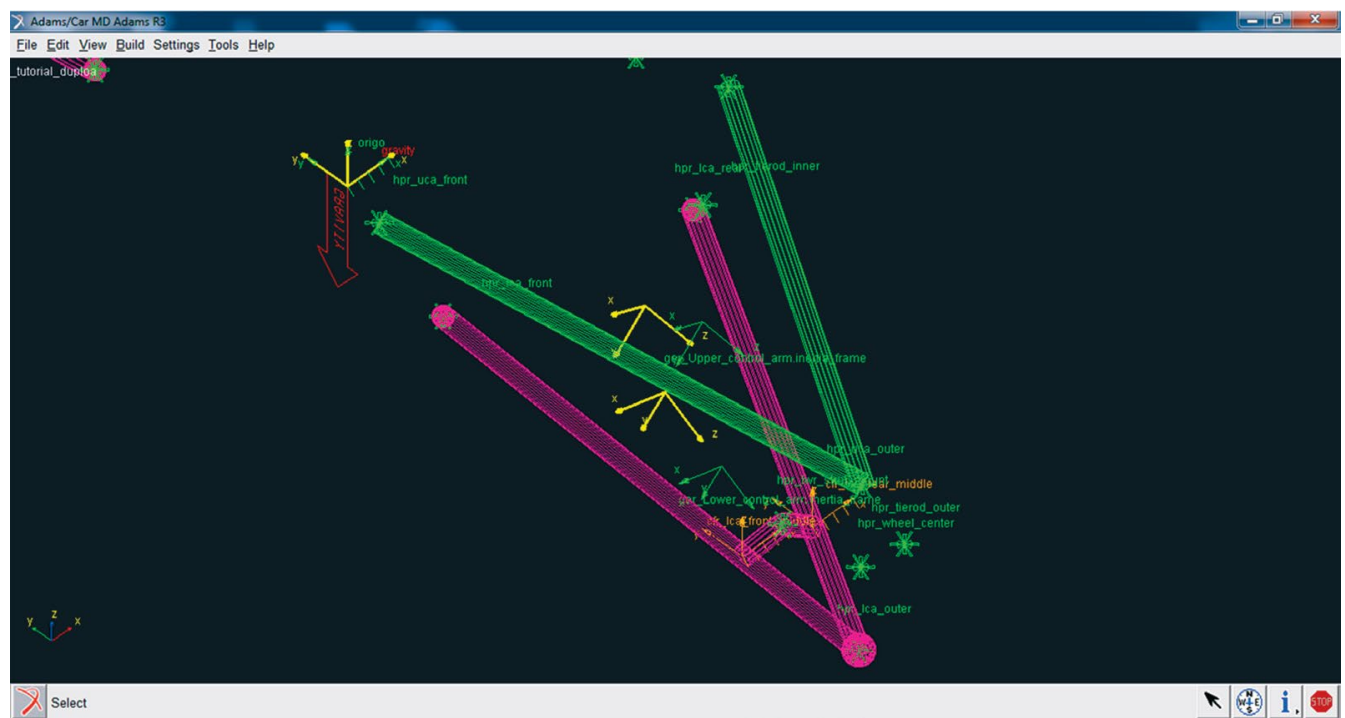

Figura 5.45 - Ilustração dos Links 5 e 6 (em verde) configurando o gel_Upper Control Arm.

- $\quad$ Salve seu projeto. 
Nas extremidades dos Links 5 e 6, serão construídos volumes esféricos, a partir da ferramenta Ellipsoid. Para tanto:

- Acesse o menu Build Geometry $\rightarrow$ Ellipsoid $\rightarrow$ New (Figura 5.46).

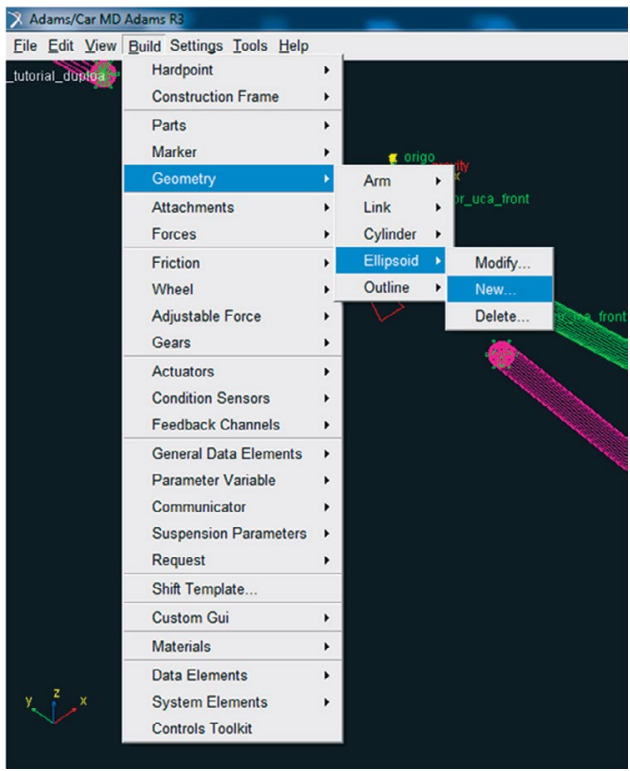

Figura 5.46 - Janela destacando as opções para criação da geometria esférica nas extremidades dos links da bandeja superior.

- Preencha a janela Create Ellipsoid Geometry, de acordo com a Figura 5.47.

- Clique em Apply.

\begin{tabular}{|c|c|c|}
\hline \multicolumn{3}{|c|}{ C Create Ellipsoid Geometry } \\
\hline \multirow{3}{*}{$\begin{array}{l}\text { Ellipsoid Name } \\
\text { Coordinate Reference } \\
\text { Method }\end{array}$} & \multicolumn{2}{|l|}{ Esf4 } \\
\hline & \multicolumn{2}{|c|}{._tutorial_duploa.ground.hpl_uca_front } \\
\hline & by entering size & 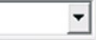 \\
\hline General Part & \multicolumn{2}{|c|}{ 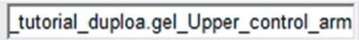 } \\
\hline X Radius & \multicolumn{2}{|l|}{10} \\
\hline Y Radius & \multicolumn{2}{|l|}{10} \\
\hline Z Radius & \multicolumn{2}{|l|}{10} \\
\hline Color & green & \\
\hline \multicolumn{3}{|c|}{ V Calculate Mass Properties of General Part } \\
\hline Density & \multicolumn{2}{|c|}{ (- Material $C$ User Entered } \\
\hline Material Type & \multicolumn{2}{|l|}{ steel $\quad=$} \\
\hline 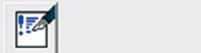 & Apply & Cancel \\
\hline
\end{tabular}

Figura 5.47 - Preenchimento da caixa de diálogo para criação da quarta esfera. 
- Preencha a janela Create Ellipsoid Geometry, de acordo com a Figura 5.48 .

- $\quad$ Clique em Apply.

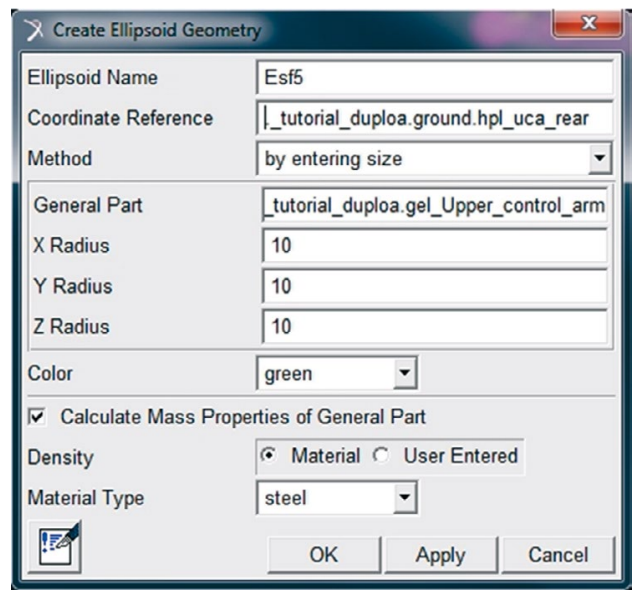

Figura 5.48 - Preenchimento da caixa de diálogo para criação da quinta esfera.

- Para a última esfera, preencha a janela Create Ellipsoid Geometry, de acordo com a Figura 5.49.

- $\quad$ Clique em $O K$.

\begin{tabular}{|c|c|c|}
\hline \multicolumn{3}{|c|}{ Create Ellipsoid Geometry } \\
\hline \multirow{3}{*}{$\begin{array}{l}\text { Ellipsoid Name } \\
\text { Coordinate Reference } \\
\text { Method }\end{array}$} & \multicolumn{2}{|l|}{ Esf6 } \\
\hline & \multicolumn{2}{|c|}{ __tutorial_duploa.ground.hpl_uca_outer } \\
\hline & by entering size & - \\
\hline General Part & \multicolumn{2}{|c|}{ tutorial_duploa.gel_Upper_control_arm } \\
\hline X Radius & \multicolumn{2}{|l|}{15} \\
\hline Y Radius & \multicolumn{2}{|l|}{15} \\
\hline Z Radius & \multicolumn{2}{|l|}{15} \\
\hline Color & green & \\
\hline \multicolumn{3}{|c|}{$\sqrt{\nabla}$ Calculate Mass Properties of General Part } \\
\hline Density & \multicolumn{2}{|c|}{ (- Material $C$ User Entered } \\
\hline Material Type & \multicolumn{2}{|l|}{ steel $\quad \nabla$} \\
\hline PEd & Apply & Cancel \\
\hline
\end{tabular}

Figura 5.49 - Preenchimento da caixa de diálogo para criação da sexta esfera.

A Figura 5.50 mostra, em verde, as três esferas criadas. 


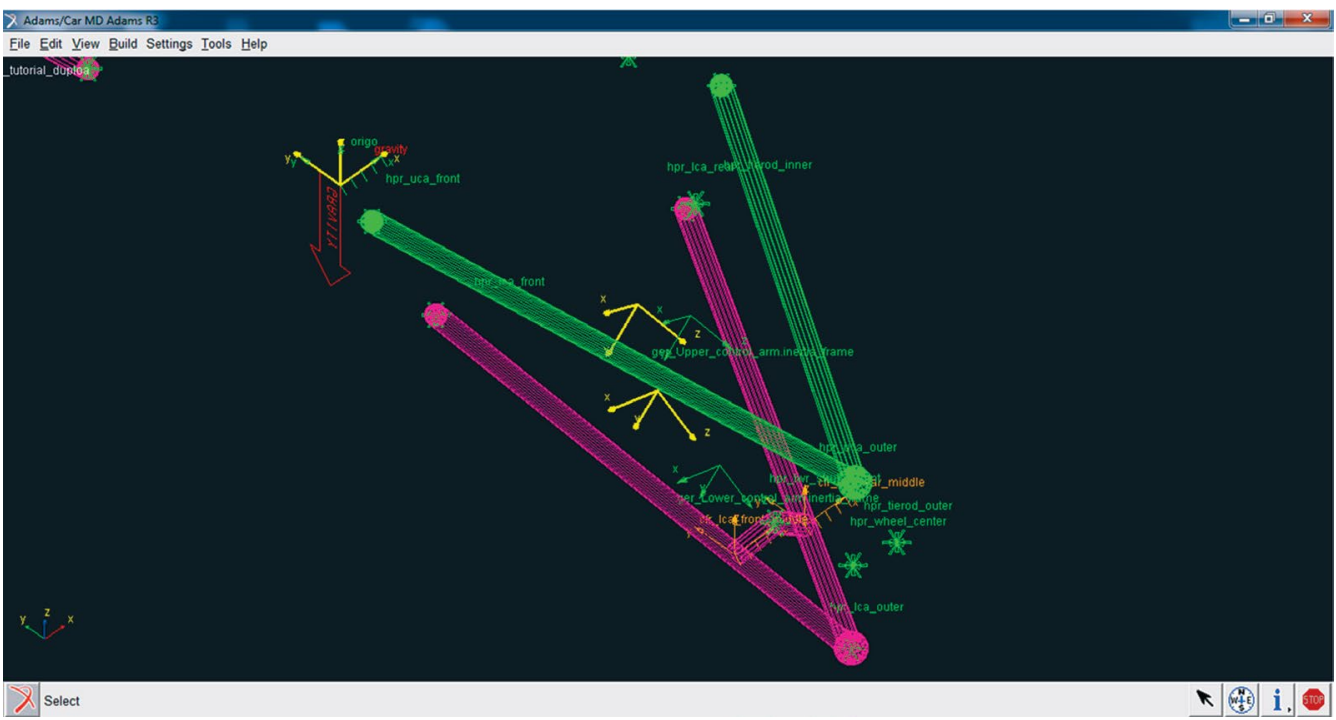

Figura 5.50 - Ilustração das esferas nas extremidades do Upper Control Arm.

- Salve seu projeto.

- Aproxime a imagem de um dos lados da suspensão, pressionando o botão direito do mouse em qualquer ponto da tela e selecionando a opção Zoom In/Out.

- Na tela principal do software clique com o botão direito do mouse sobre o General Part "gel_Upper_Control_Arm".

- Clique em Modify, Figura5.51 e com a caixa de diálogo resultante da Figura 5.52 clique sobre o ícone calculadora.

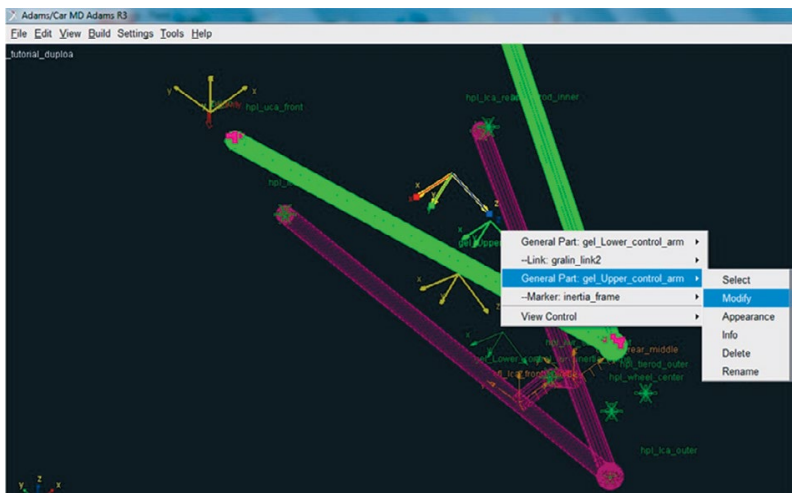

Figura 5.51 - Opção para atualização das propriedades do General Part Lower_control_arm a partir da opção Modify. 


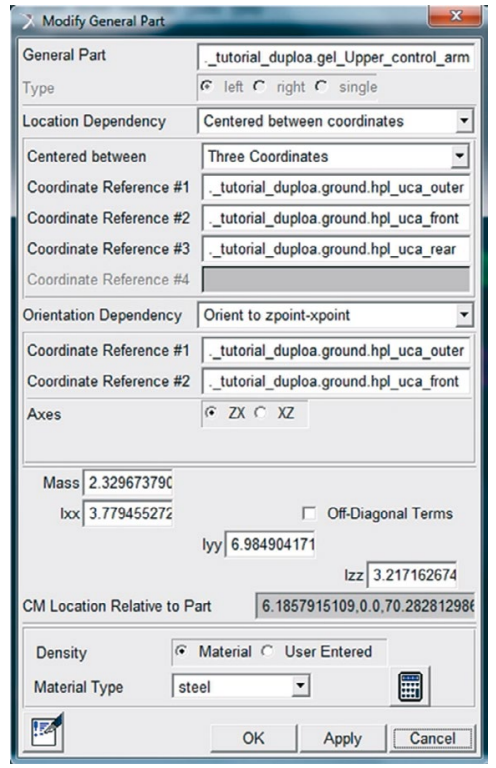

Figura 5.52 - Atualização das propriedades do General Part "gel_Upper_control_arm”.

Etapa 10 - Criação da manga de eixo (Upright)

Inicialmente, será criado o General Part associado à manga de eixo. Cabe salientar que, de acordo com a Figura5.10, haverá quatro Hardpoints associados a essa parte (Part): $h p l \_l c a \_o u t e r, h p l \_u c a \_o u t e r, h p l \_w h e e l \_c e n t e r$ e $h p l \_t i e r o d \_$ outer e, ainda, três geometrias do tipo Link. Dando continuidade à etapa, tem-se:

- Acesse o menu Build $\rightarrow$ Parts $\rightarrow$ General Part $\rightarrow$ New, conforme ilustra a Figura 5.53.

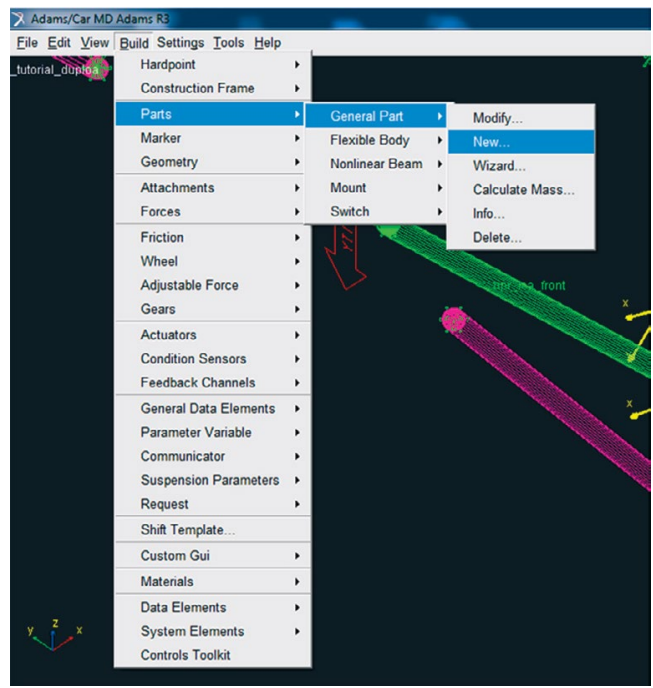

Figura 5.53 - Janela correspondente á construção do General Part "Uprigth”. 
A janela Create General Part será aberta e deverá ser preenchida, conforme ilustra a Figura 5.54.

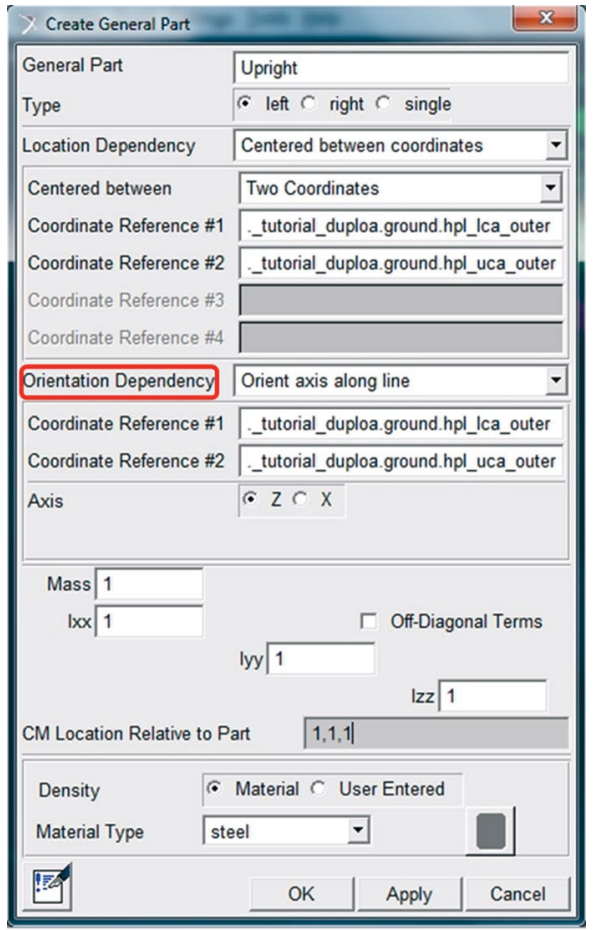

Figura 5.54 - Janela correspondente à criação do General Part "Uprigth".

\section{- $\quad$ Clique em $O K$.}

A notar: A opção Orientation Dependency, Figura 5.54, selecionada como Orient axis along line solicita a especificação de duas coordenadas. Perceba que entre os dois Hardpoints especificados pode-se imaginar um eixo de ligação, que na aba Axis, foi especificado como sendo o eixo " $Z$ ". A direção, neste caso, é da coordenada 1 até a coordenada 2.

O resultado é mostrado na Figura 5.55. 


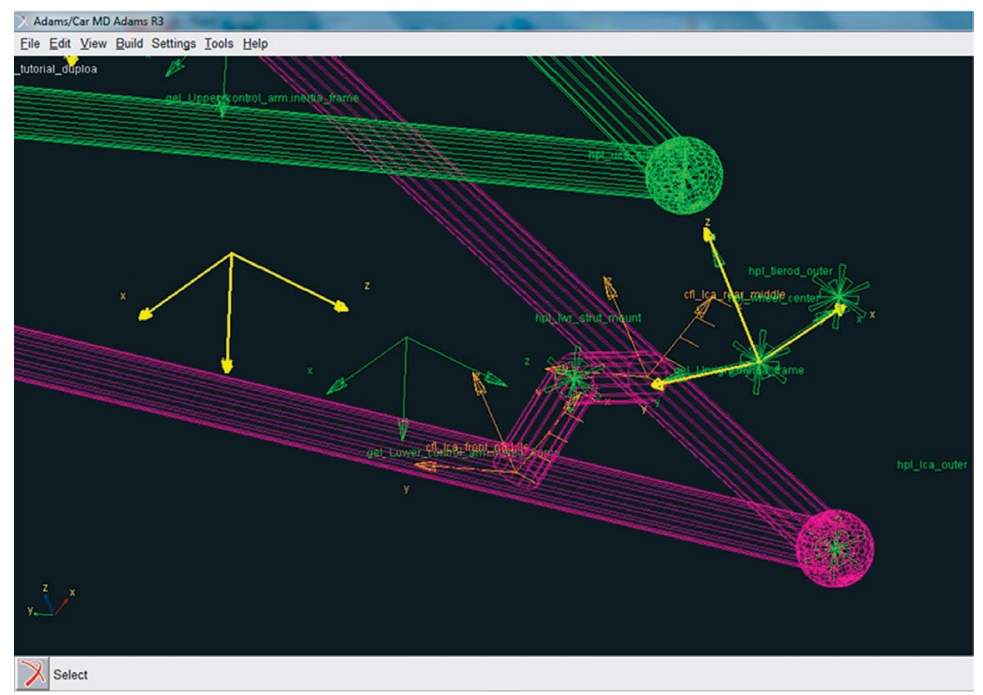

Figura 5.55 - Tela ilustrativa da criação do General Part "Upright".

Em seguida, serão construídos dois Links, que complementam a manga de eixo. Para a criação destes Links siga as instruções a seguir:

- $\quad$ Acesse o menu Build $\rightarrow$ Geometry $\rightarrow$ Link $\rightarrow$ New, conforme ilustrado na Figura 5.56.

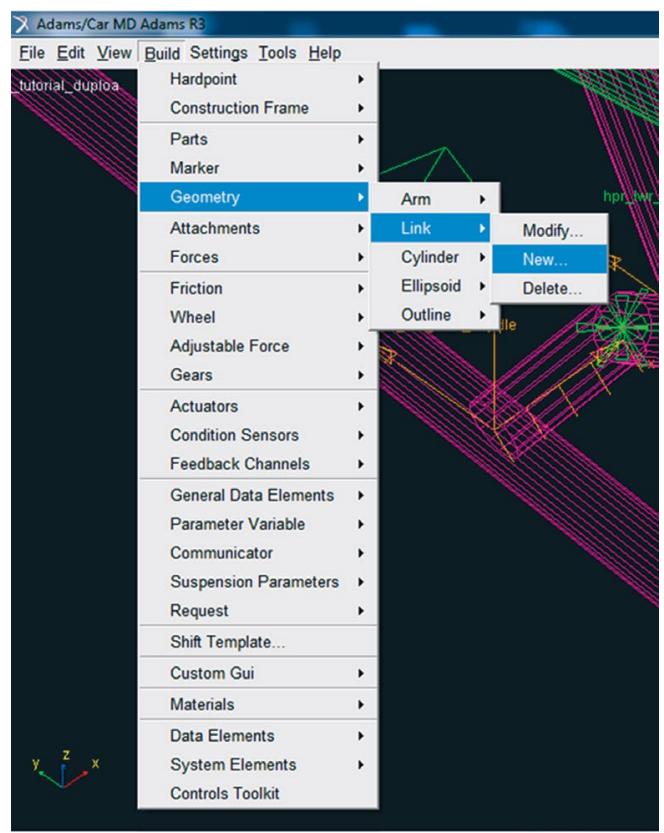

Figura 5.56 - Janela correspondente à criação da geometria Link que constituirá a manga de eixo. 
A janela Create Link Geometry será aberta e a Figura 5.57 ilustra seu preenchimento.

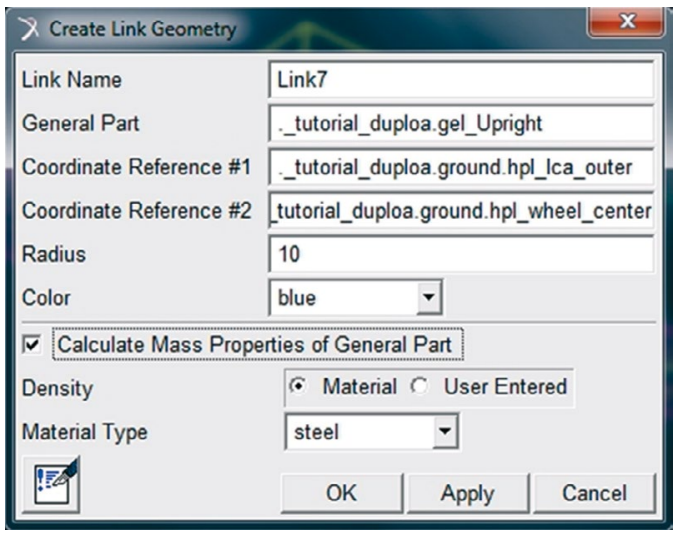

Figura 5.57 - Janela relativa à criação do primeiro Link correspondente ao General Part "Upright".

- $\quad$ Clique em Apply.

O mesmo procedimento deve ser seguido para a construção do segundo Link. A Figura 5.58 ilustra o preenchimento da janela correspondente a sua criação.

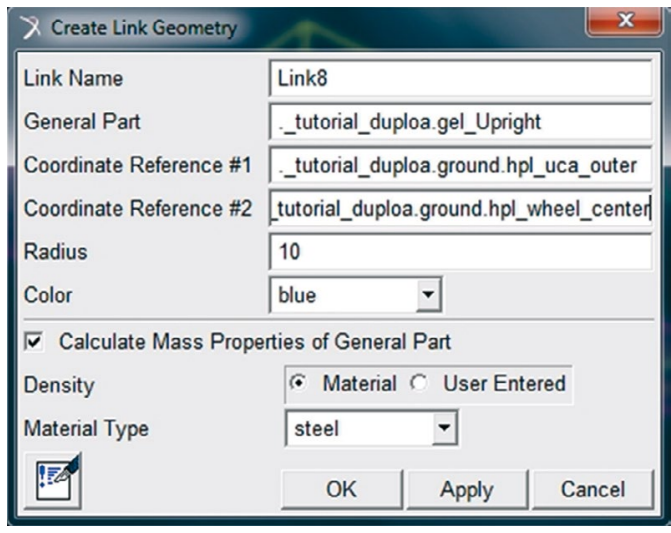

Figura 5.58 - Janela relativa à criação do segundo Link correspondente ao General Part "Upright".

- $\quad$ Clique em $O K$.

- $\quad$ Salve seu projeto.

Considerando que a manga de eixo da suspensão Duplo A, geralmente, vem acompanhada por um suporte de fixação da direção, o próximo passo será a construção de um terceiro Link, conforme ilustrado na Figura 5.59. 


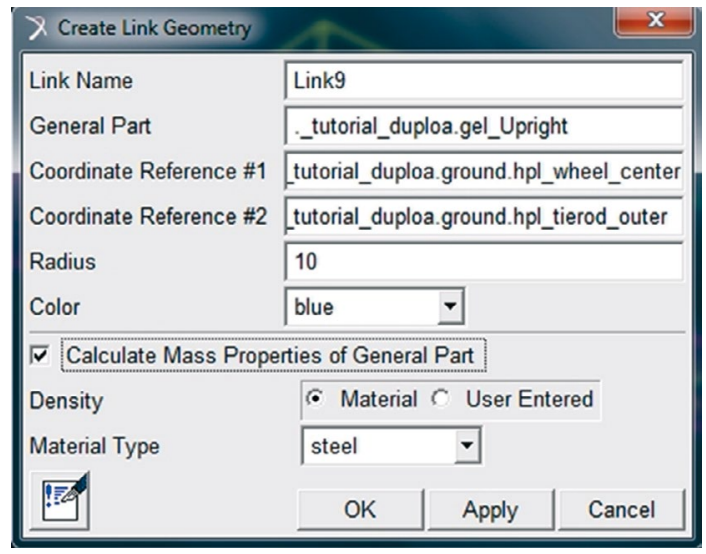

Figura 5.59 - Janela relativa à criação do segundo Link correspondente ao General Part "Upright".

- $\quad$ Clique em $O K$.

A Figura 5.60 destaca a parte General Part “Uprigth” com os três Links criados, nas Figuras 5.57, 5.58, e 5.59, em azul.

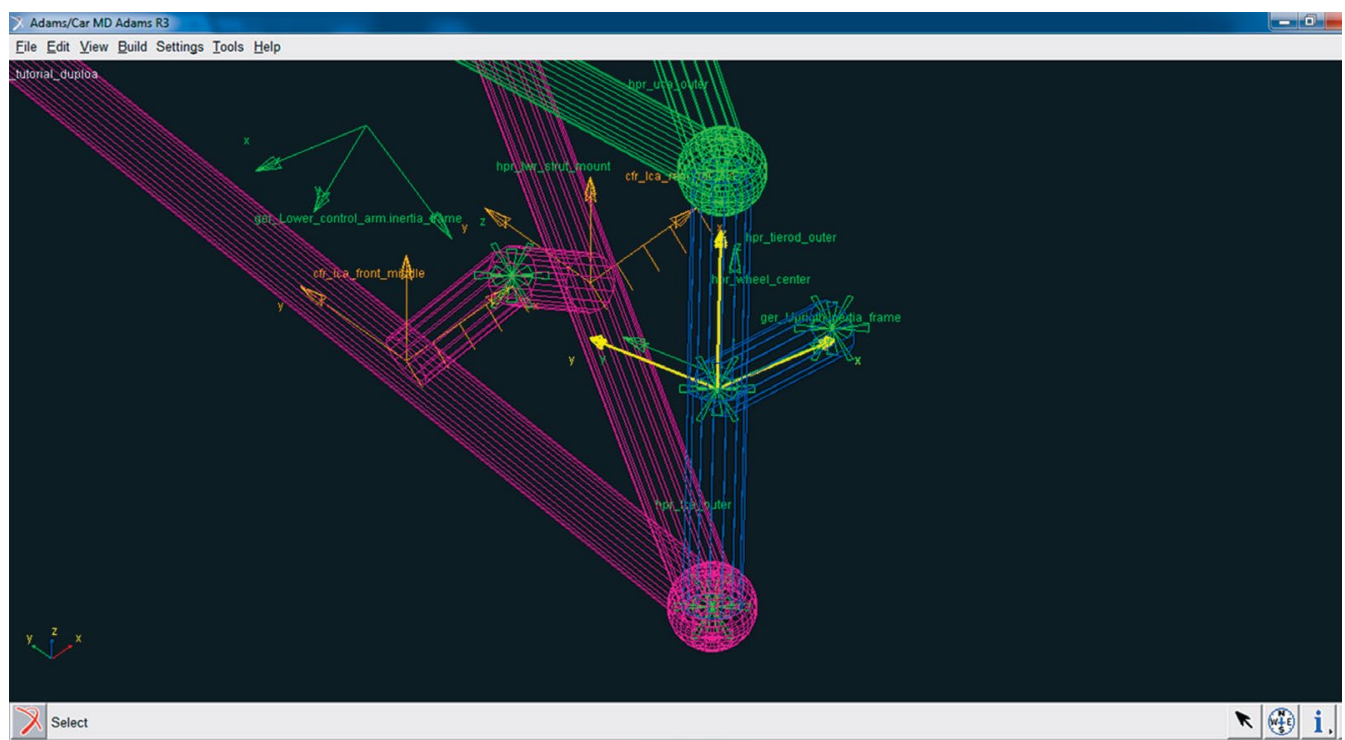

Figura 5.60 - Ilustração do General Part “Upright” associado às geometrias Links

- $\quad$ Salve seu projeto.

Etapa 11 - Criação do elemento de ancoragem do amortecedor ao chassi

Nesta etapa, será construído o elemento (corpo rígido) de fixação do conjunto mola/amortecedor ao chassi do veículo. 
Antes de criar o General Part e as geometrias relacionadas, serão criados três Construction Frames para definir a orientação da estrutura a ser criada. Para isso: - Acesse o menu Build Construction Frame $\rightarrow$ New (Figura 5.61).

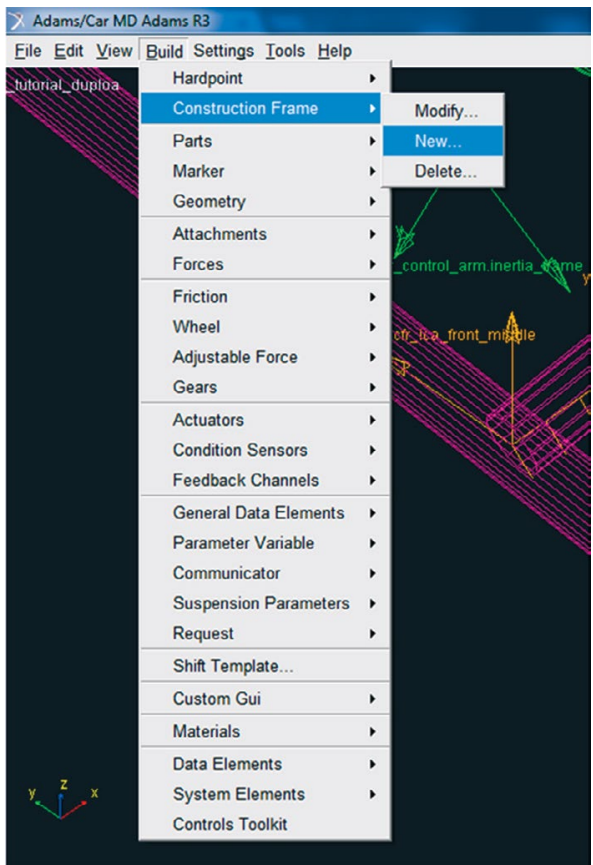

Figura 5.61 - Criação do Construction Frame.

- A janela Create Construction Frame será aberta e os campos deverão ser preenchidos, conforme a Figura 5.62.

Note que esse Construction Frame será criado, utilizando-se, como coordenada de referência, o Hardpoint "hpl_lwr_strut_mount".

- Clique em Apply. 


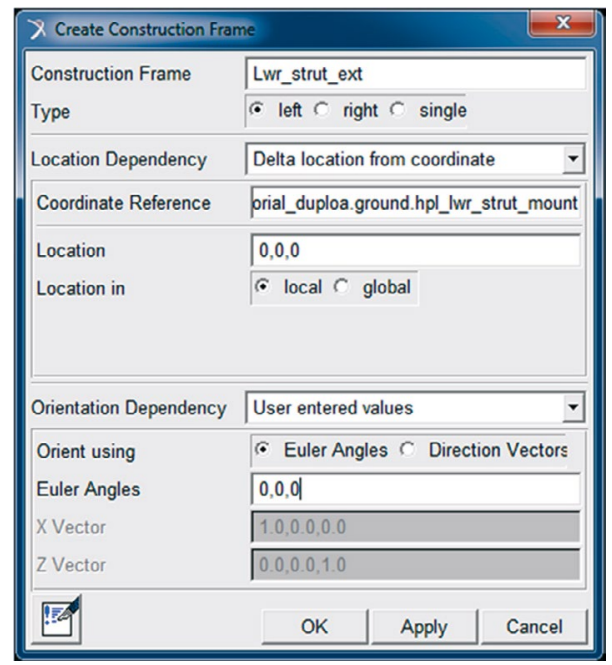

Figura 5.62 - Janela relativa à criação do Construction Frame "Lwr_strut_ext".

\section{A Figura 5.63 ilustra o Construction Frame construído.}

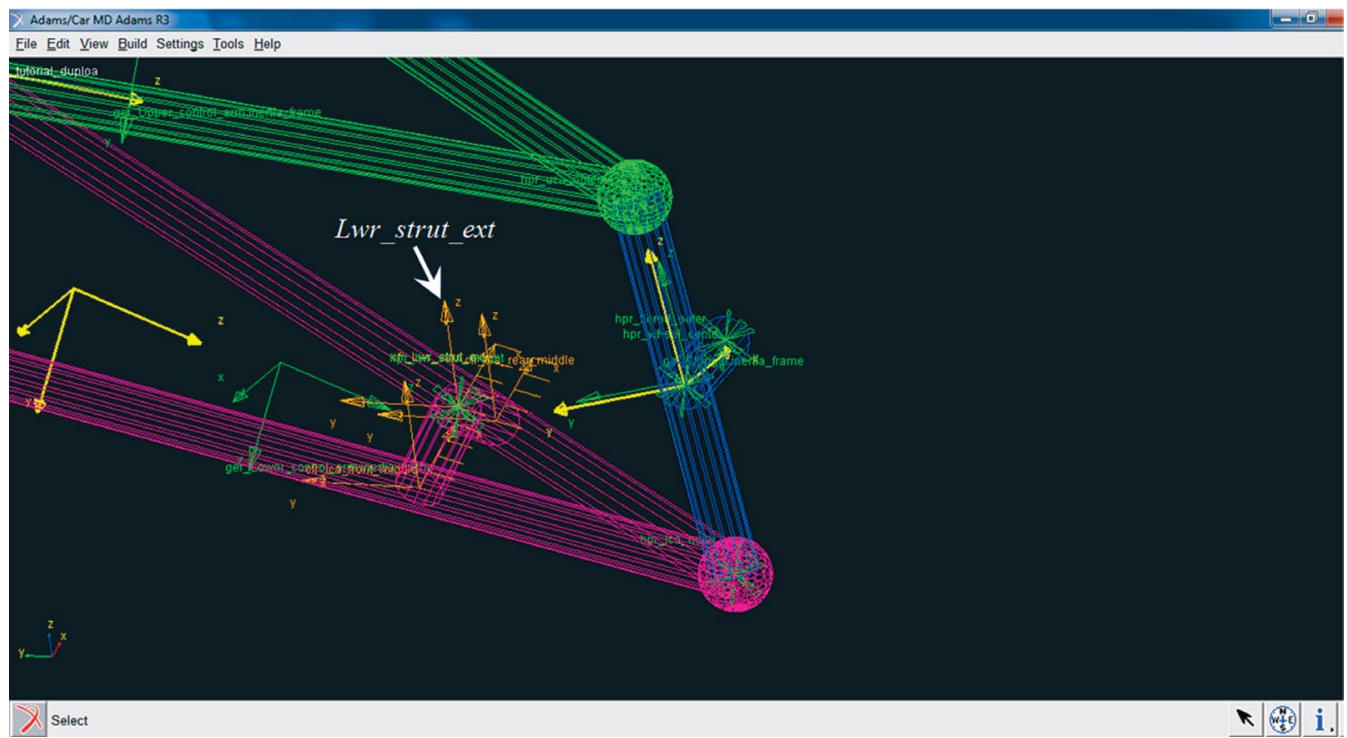

Figura 5.63 - Tela ilustrativa do Construction Frame "Lwr_strut_ext".

As janelas devem ser preenchidas, conforme mostram as Figuras 5.64 e 5.66. - Clique novamente em Apply após a criação do segundo Construction Frame “Top_mount_ext” e em OK, após concluir o terceiro Lwr spring_seat. 
As telas ilustrativas de cada uma das operações são mostradas nas Figuras 5.65 e 5.67 .

\begin{tabular}{|c|c|c|}
\hline \multicolumn{2}{|l|}{ Create Construction Frame } & $x$ \\
\hline Construction Frame & Top_mount_ext & \\
\hline Type & (- left $C$ right $C$ single & \\
\hline Location Dependency & Delta location from coordinate & $\nabla$ \\
\hline Coordinate Reference & -_tutorial_duploa.ground.hpl__ & p_mount \\
\hline Location & $0,0,0$ & \\
\hline Location in & - local 5 global & \\
\hline Orientation Dependency & User entered values & - \\
\hline Orient using & - Euler Angles $\subset$ Directio & Vectors \\
\hline Euler Angles & $0,0,0$ & \\
\hline $\mathrm{X}$ Vector & & \\
\hline Z Vector & & \\
\hline 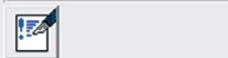 & Apply & Cancel \\
\hline
\end{tabular}

Figura 5.64 - Janela relativa à construção do Construction Frame "Top_ mount_ext”.

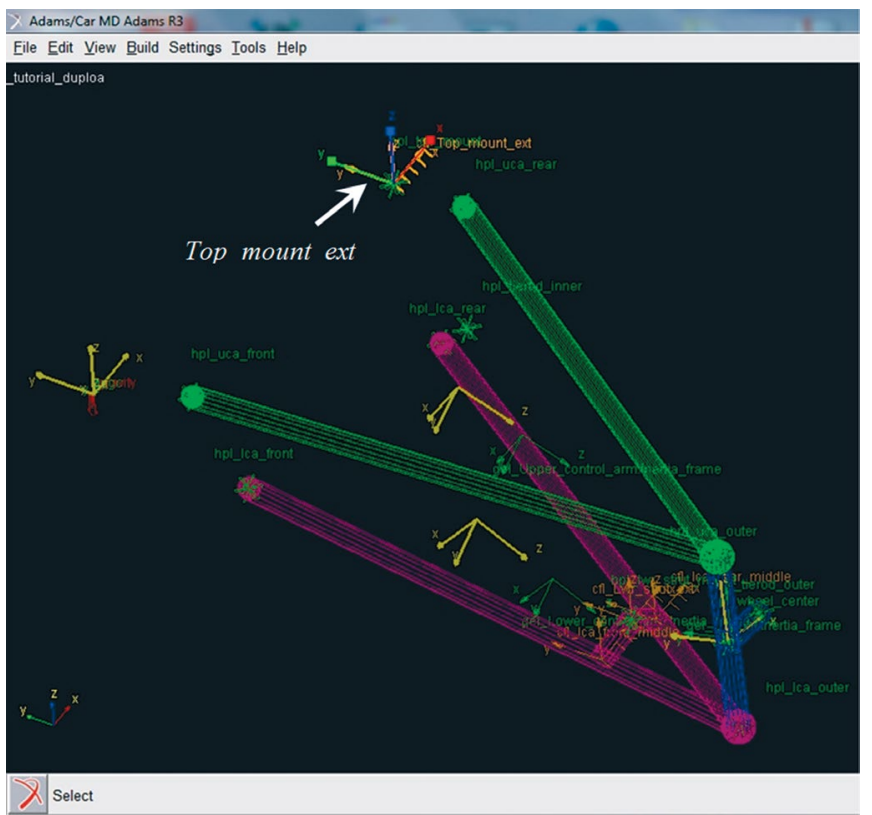

Figura 5.65 - Tela ilustrativa do Construction Frame "Top_mount_ext". 


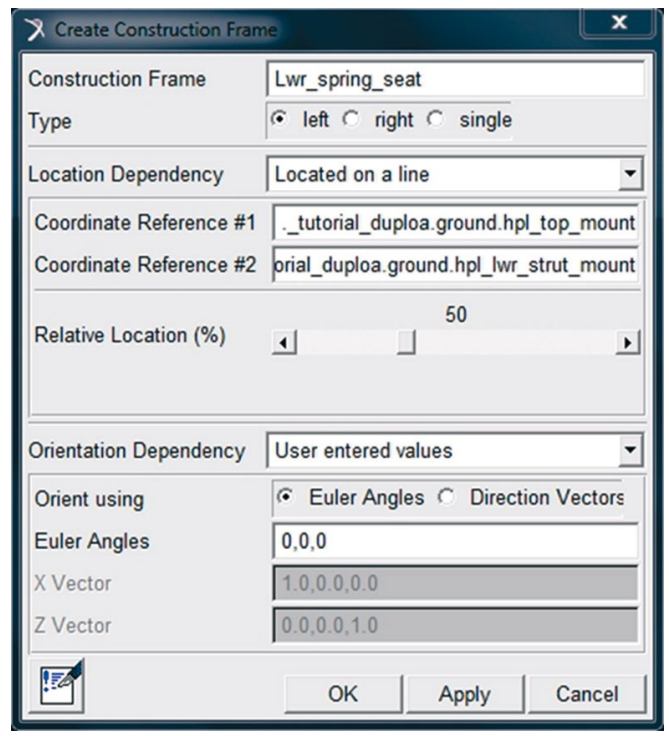

Figura 5.66 - Janela relativa à construção do Construction Frame "Lwr_spring_seat".

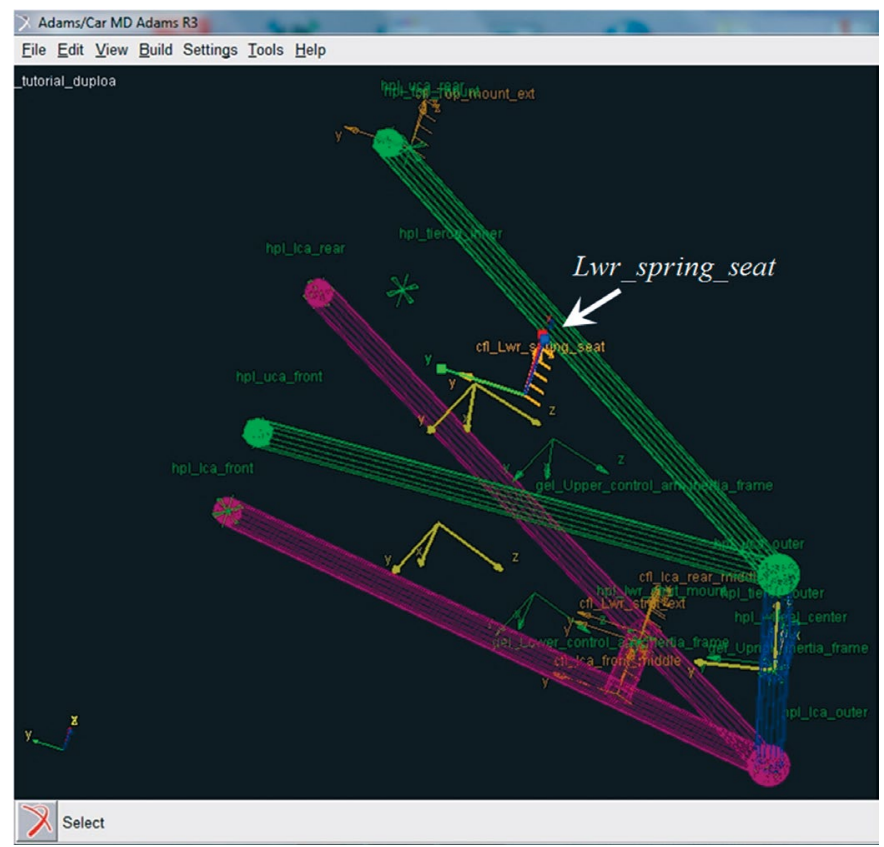

Figura 5.67 - Tela ilustrativa do Construction Frame "Lwr_spring_seat".

Note que a construção dos três Construction Frames definiram as coordenadas por onde a linha de construção do conjunto mola/amortecedor irá passar. Os 
dois primeiros fixaram as extremidades, enquanto o último, posicionado no meio do segmento de reta (Relative Location igual a 50\%), complementou a orientação. - Salve seu projeto.

Após a construção dos Construction Frames, a próxima etapa consiste na criação do General Part "Upper Strut". Neste serão definidos os parâmetros da mola e do amortecedor da suspensão. Para tanto:

- Acesse o menu Build $\rightarrow$ Parts $\rightarrow$ General Part $\rightarrow$ New, conforme a Figura 5.68 .

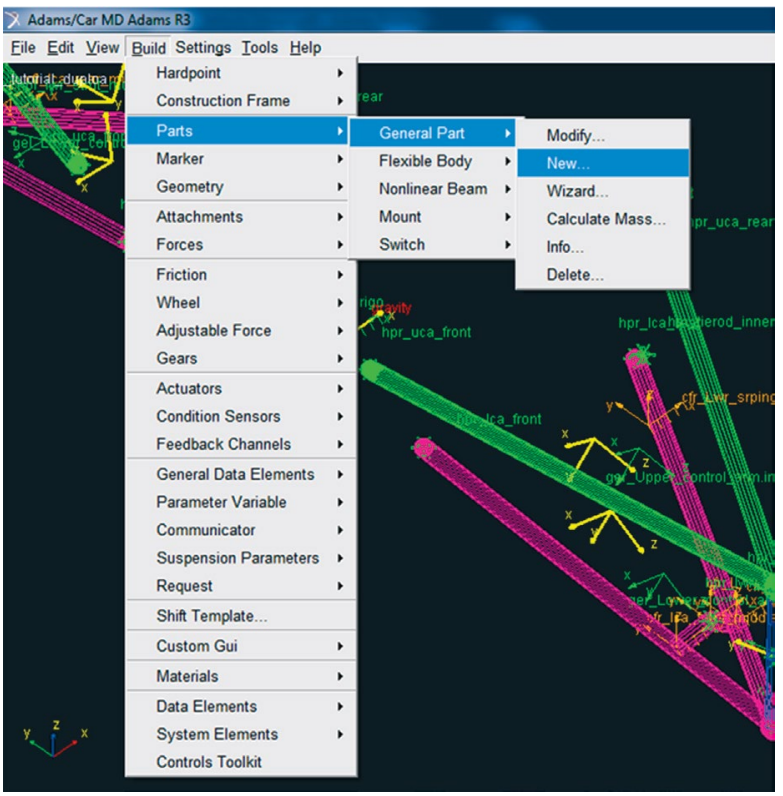

Figura 5.68 - Tela correspondente à opção de construção do General Part "Upper-strut".

A janela Create General Part será aberta e seus campos deverão ser preenchidos, conforme a Figura 5.69.

- Ao finalizar o preenchimento, clique em $O K$. 


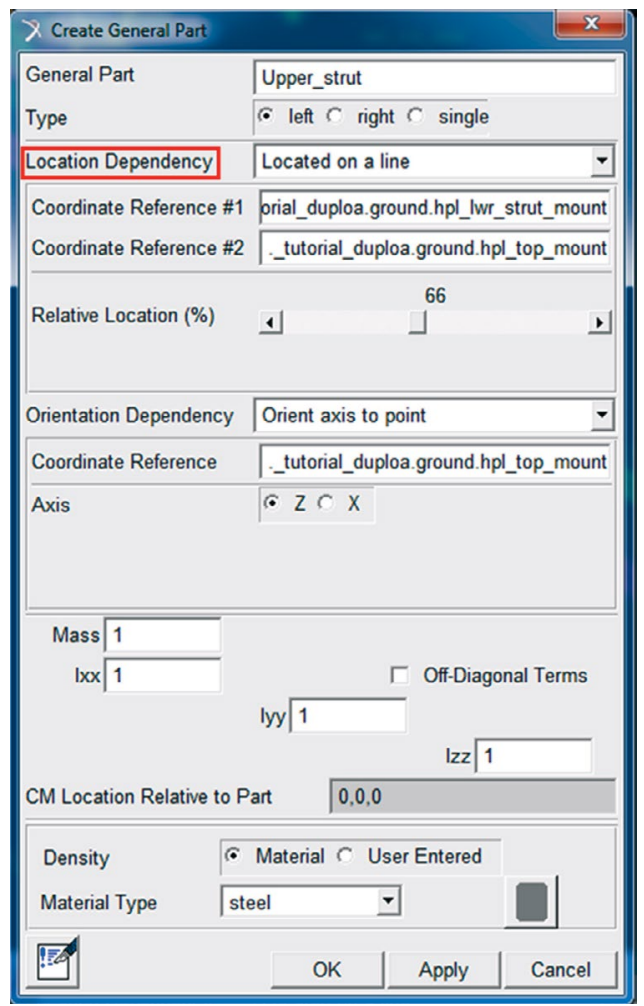

Figura 5.69 - Janela relativa à construção do General Part "Upper_strut".

A notar: A escolha da aba Location Dependency como Located on a Line, Figura 5.69, permite que o General Part "Upper_strut" se situe a $66 \%$ da distância entre os dois Hardpoints especificados como coordenadas \#1 e \#2, medido relativamente à coordenada \#1.

A Figura 5.70 ilustra a criação do referido General Part. 


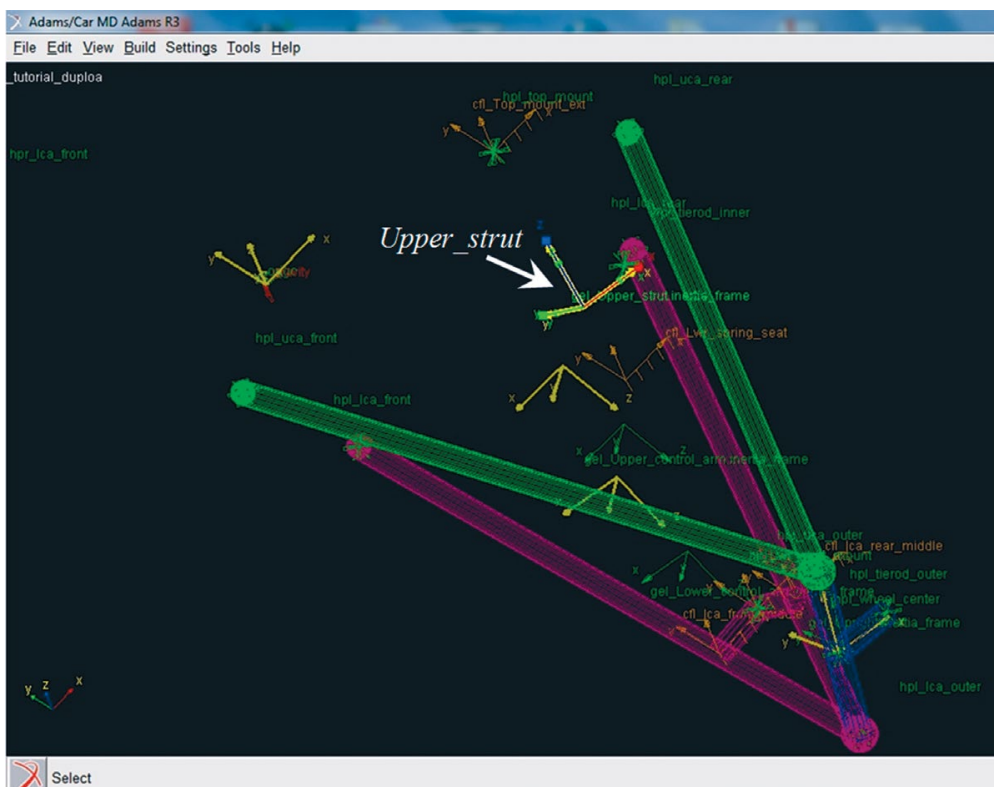

Figura 5.70 - Tela ilustrativa do General Part "Upper_strut”.

- Crie outro General Part que, juntamente com o General Part "Upper_ strut", representará o comportamento do amortecedor e da mola. Para criá-lo acesse o menu Build $\rightarrow$ Parts $\rightarrow$ General Part $\rightarrow$ New (Figura 5.71).

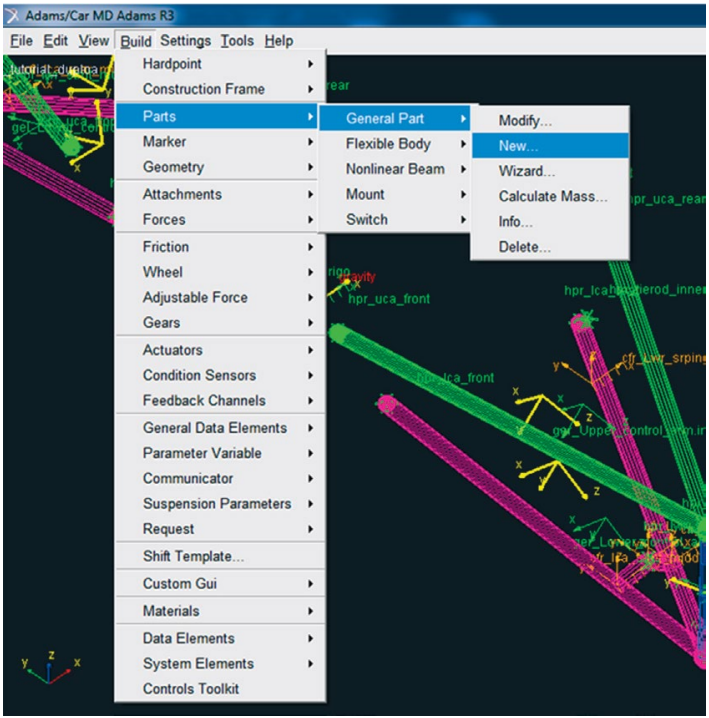

Figura 5.71 - Tela correspondente à opção de construção do General Part. 
- A janela Create General Part será aberta e seus campos deverão ser preenchidos conforme a Figura 5.72.

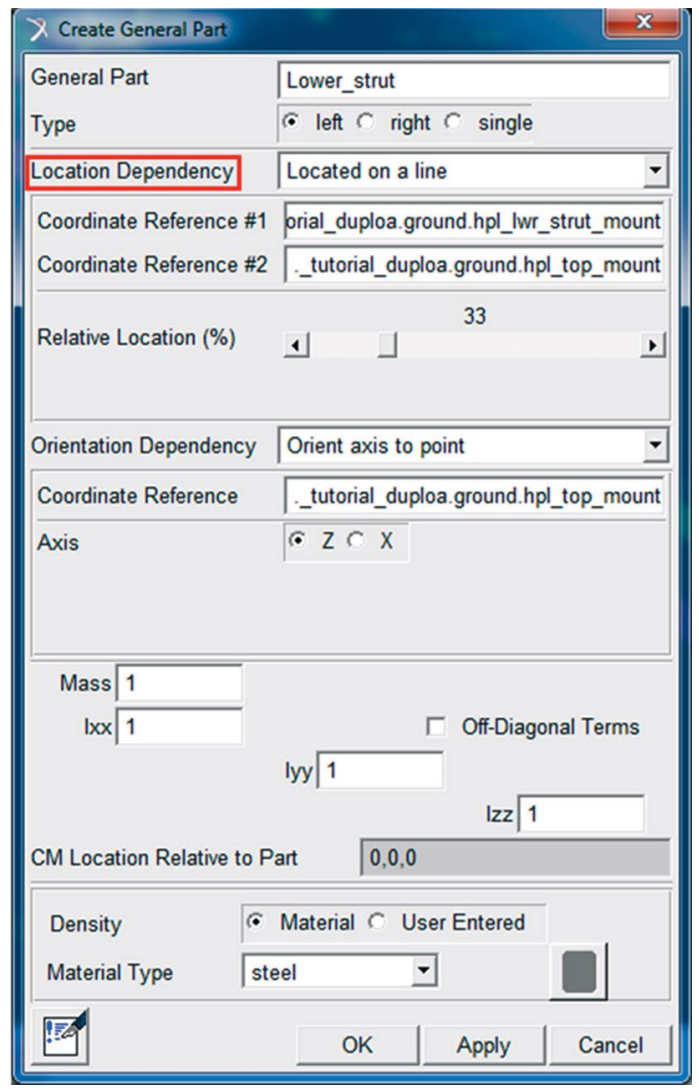

Figura 5.72 - Janela relativa à construção do General Part "Lower_strut".

A notar: A escolha da aba Location Dependency como "Located on a Line", Figura 5.72, permite que o General Part "Lower_strut" se situe a 33\% da distância entre os dois Hardpoints especificados como coordenadas \#1 $e$ \#2, medido relativamente à coordenada \#1.

- $\quad$ Clique em $O K$.

A Figura 5.73 ilustra o General Part "Lower_strut". 


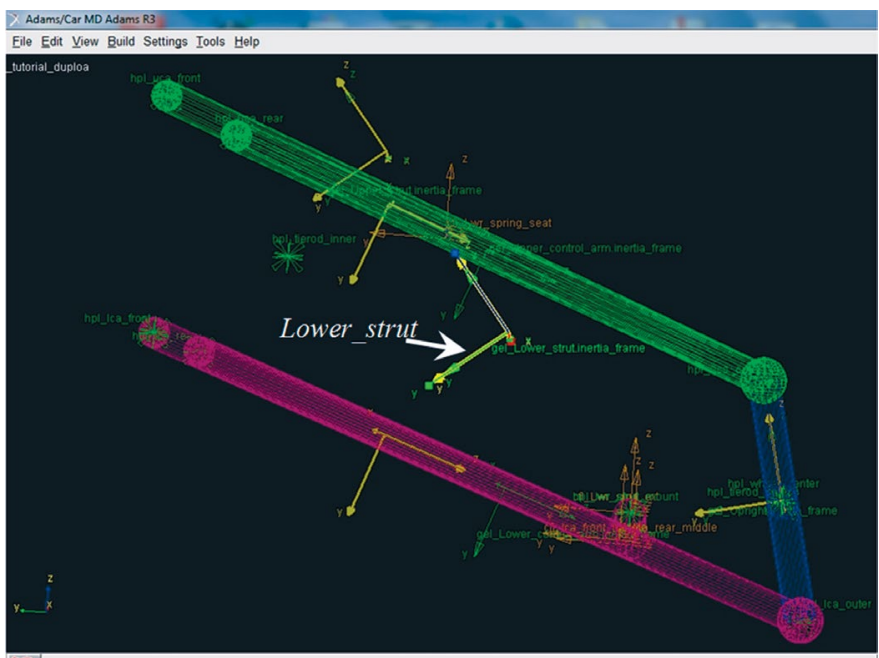

$\lambda$ select

Figura 5.73 - Tela ilustrativa do General Part "Lower_Strut".

\section{Etapa 12 - Definindo parâmetros da suspensão}

Nesta etapa, serão definidos os parâmetros de Camber, Toe (convergência) e Cáster da suspensão. Para isso:

- Acesse o menu Build $\rightarrow$ Suspension Parameters $\rightarrow$ Toe/Camber Values $\rightarrow$ Set (Figura 5.74).

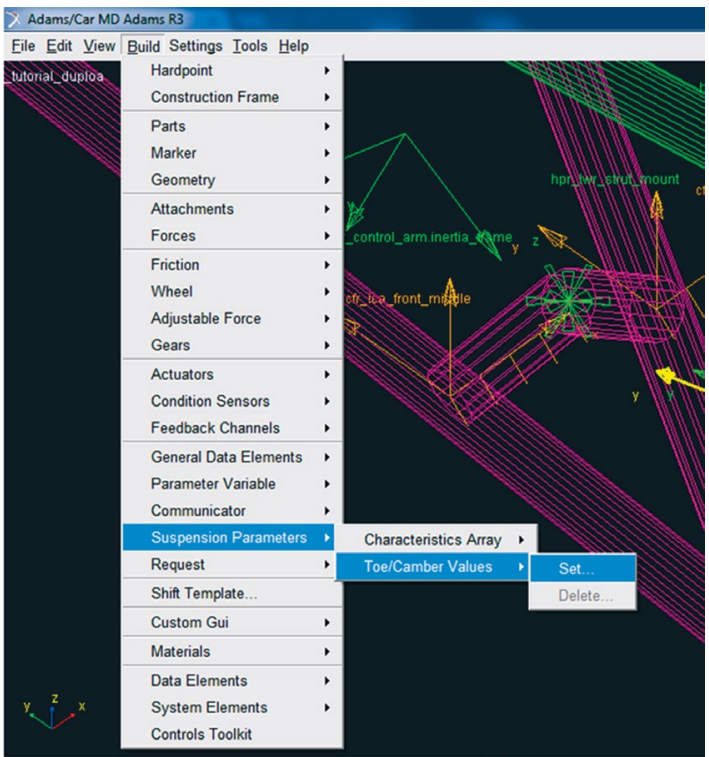

Figura 5.74 - Definição de parâmetros de Toe e Camber da suspensão. 
A janela Set Toe \& Camber Values será aberta e os valores destes parâmetros deverão ser inseridos nos campos disponíveis, de acordo com o projeto. Note que os valores requeridos se referem a ambos os lados: esquerdo (Left) e direito (Right) (Figura 5.75).

- Após preenchimento, clique em $O K$.

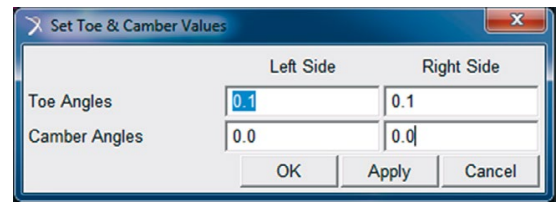

Figura 5.75 - Janela de inserção dos valores de Toe e Camber da suspensão Duplo A.

A notar: O preenchimento da caixa de diálogo mostrada na Figura 5.75 dá origem a dois parâmetros da geometria da suspensão que serão denominados: pvl_Toe_Angle $e$ pvl_Camber_Angle. Esses parâmetros pertencem a uma classe denominada Parameter Variables (pv) e serão utilizados na construção do Construction Frame "upright_ spindle_inr", Figura 5.79 - Etapa 13.

A definição do valor de Caster da suspensão se dá a partir do preenchimento das informações da Figura 5.77. Para isso:

- Acesse o menu Build $\rightarrow$ Suspension Parameter $\rightarrow$ Characteristics Array $\rightarrow$ Set (Figura 5.76).

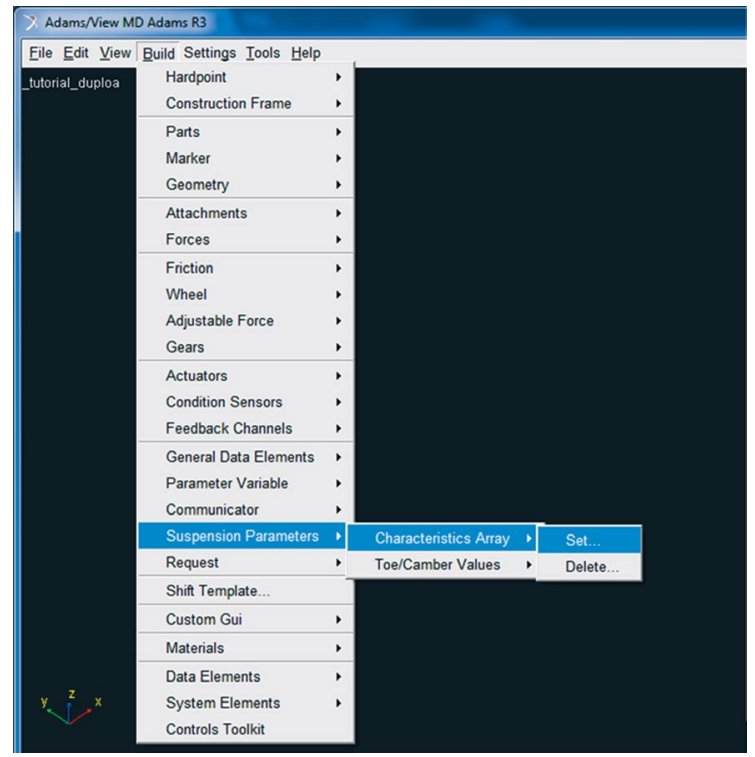

Figura 5.76 - Opção de definição do Caster da suspensão Duplo A. 
- Preencha a janela Suspension Parameters Array, conforme ilustrado na Figura 5.77.

- Ao final, clique em $O K$.

Perceba que a suspensão foi definida como independente na aba Suspension Type, selecionando-se a opção a partir da seta à direita da aba. O eixo de direção (Steer axis) é definido pelos Hardpoints inseridos nas abas I Coordinate Reference, J Coordinate Reference. I Part e J Part são preenchidos com informações a partir de General Part.

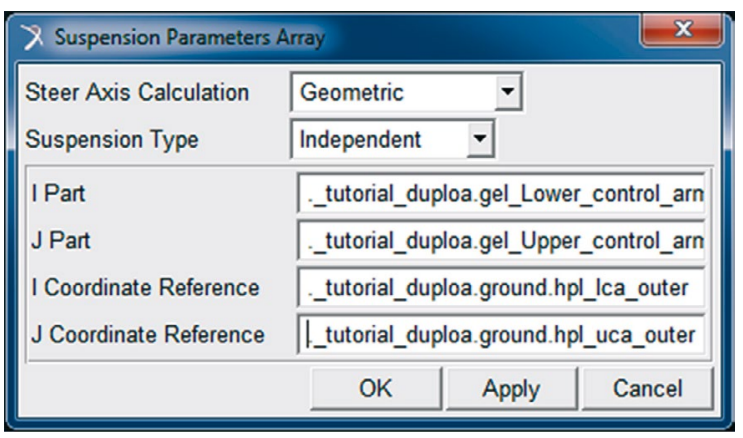

Figura 5.77 - Definição do Caster da suspensão.

\section{Etapa 13 - Construção da geometria relacionada ao cubo de roda}

Para construção da geometria (Geometry), referente ao cubo de roda, é necessário, primeiramente, criar um novo Construction Frame, o qual irá fornecer a orientação das variáveis Toe e Camber. O preenchimento do campo Orientation Dependancy, assinalado na Figura 5.79, garante que as características estabelecidas na Etapa 12 sejam atendidas. Assim:

- Acesse o menu Build $\rightarrow$ Construction Frame $\rightarrow$ New (Figura 5.78). 


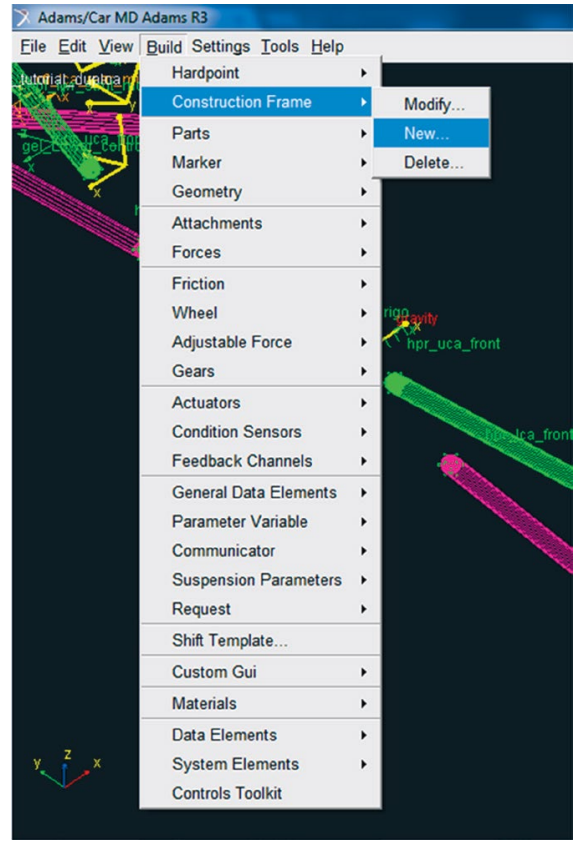

Figura 5.78 - Construção do Construction Frame relacionado ao cubo da roda.

A janela Create Construction Frame será aberta e os campos deverão ser preenchidos, conforme mostrado na Figura 5.79. Observe que o Construction Frame estará localizado no $h p l \_W h e e l \_C e n t e r$ e terá sua orientação definida pelas variáveis Toe e Camber declaradas anteriormente.

- $\quad$ Finalizado o preenchimento, clique em OK.

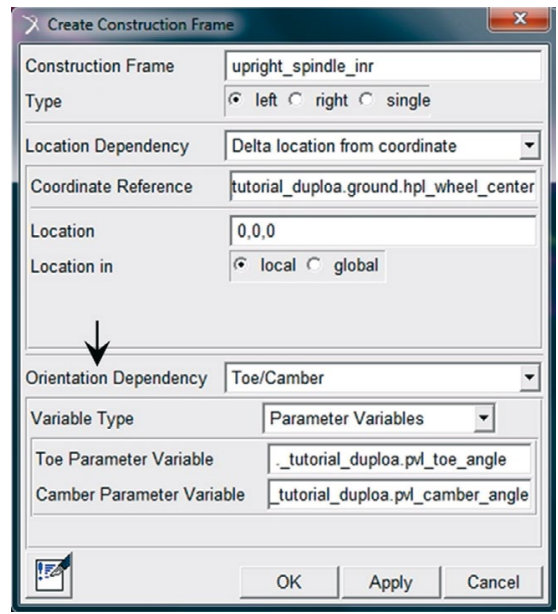

Figura 5.79 - Definindo a posição da Construction Frame. 
A Figura 5.80 ilustra o Construction Frame "Upright_spindle_inr".

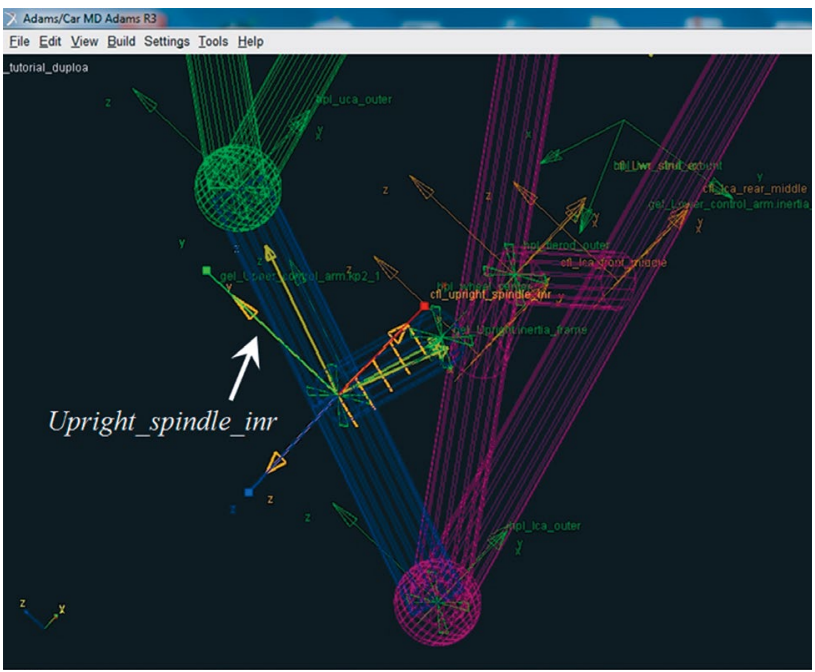

Figura 5.80 - Tela ilustrativa do Construction Frame "upright_spindle_inr".

Após esta ação, parte-se para a construção da geometria. Para isso:

- Acesse o menu Build $\rightarrow$ Geometry $\rightarrow$ Cylinder $\rightarrow$ New, associado ao Construcrion Frame (Figura 5.81).

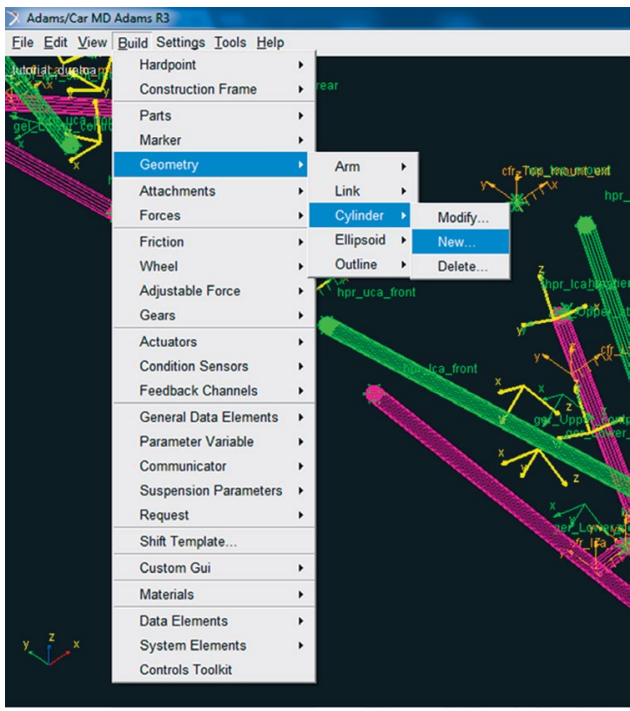

Figura 5.81 - Criação de uma geometria do tipo Cylinder. 
A notar: A diferença entre as geometrias Link $e$ Cylinder são sutis visualmente, diferenciando-se apenas no modo de suas construções. Enquanto o Link necessita da definição de dois hardpoints $e$ do raio, o Cylinder requer a definição de um Construction Frame. O eixo z do Construction Frame define o eixo longitudinal do cylinder, e seu comprimento é fornecido tanto no sentido positivo quanto negativo do eixo z. O raio do cylinder também é um parâmetro requisitado.

A janela Create Cylinder Geometry será aberta e os campos a serem preenchidos estão ilustrados na Figura 5.82. Note que para construção de uma geometria cilíndrica é necessário definir um Construction Frame ( $3^{\circ}$ campo) o qual já foi criado anteriormente nesta mesma etapa.

- $\quad$ Clique em $O K$.

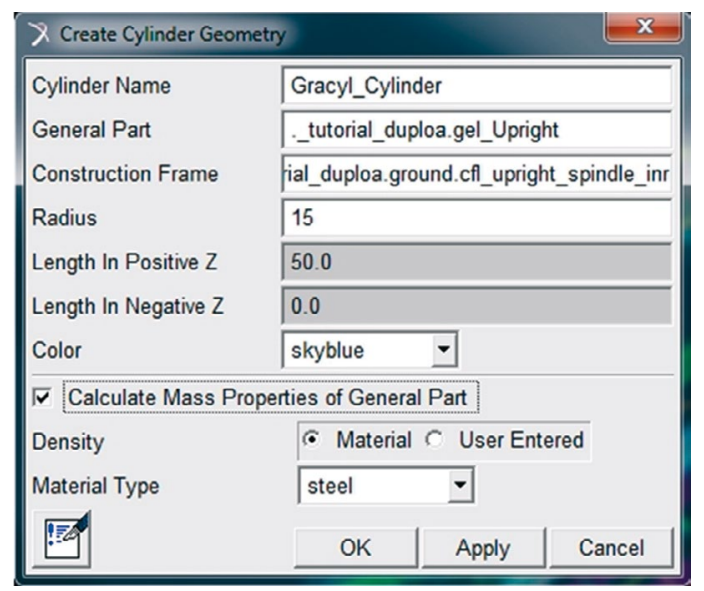

Figura 5.82 - Janela relativa à construção da geometria Cylinder referente ao cubo da roda.

A Figura 5.83 ilustra o cilindro representativo do cubo da roda. 


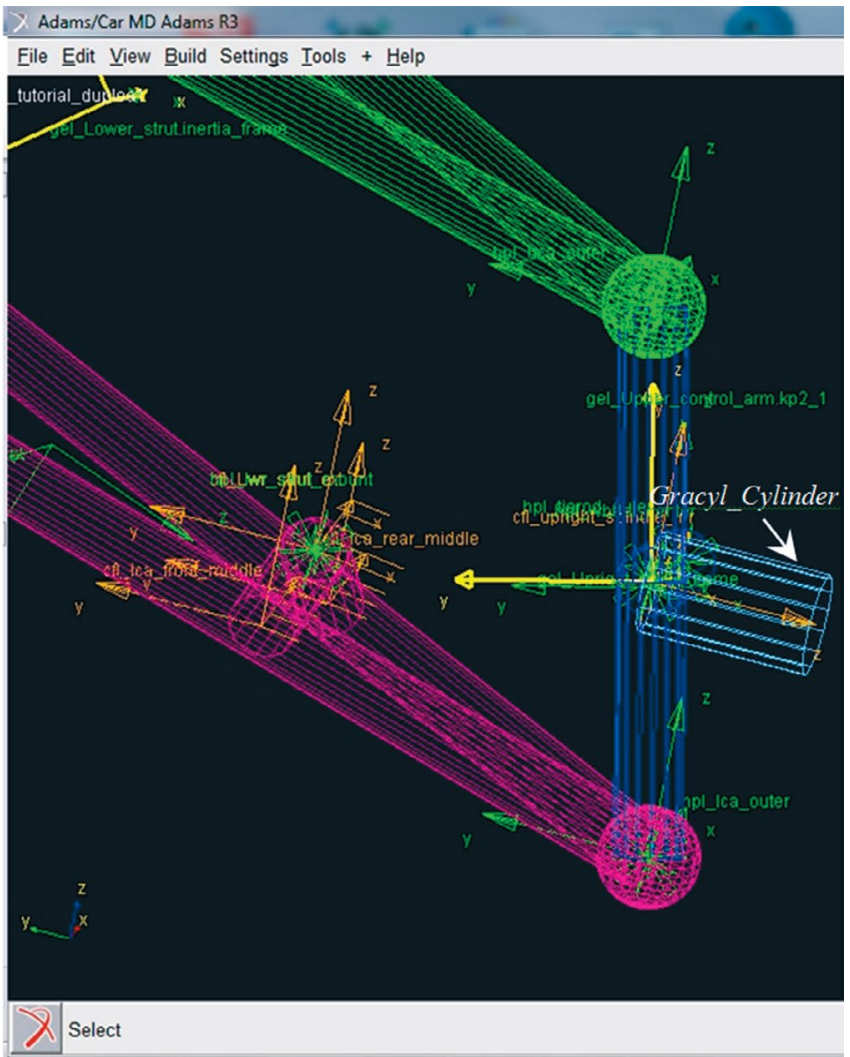

Figura 5.83 - Tela ilustrativa do cilindro Gracyl_Cylinder.

Etapa 14 - Construção do amortecedor (Damper)

- Para a criação do amortecedor, acesse o menu Build $\rightarrow$ Forces $\rightarrow$ Damper $\rightarrow$ New (Figura 5.84). 


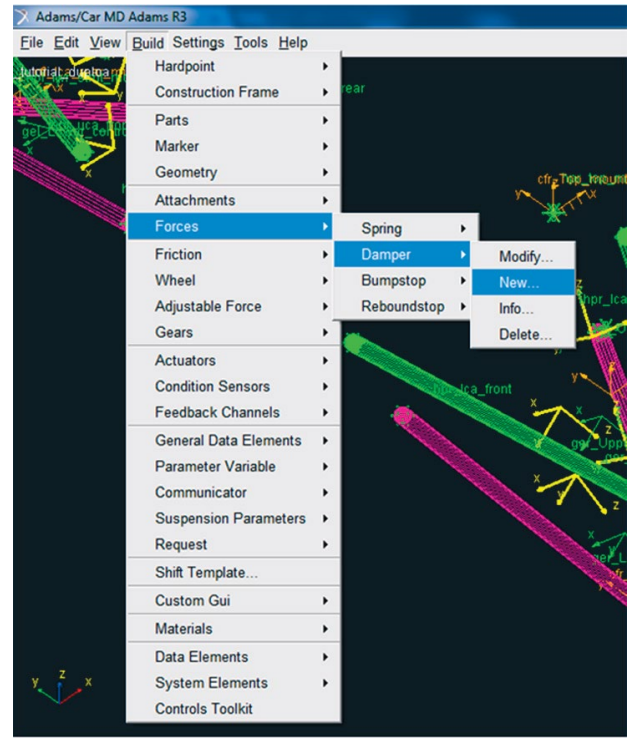

Figura 5.84 - Definição do amortecedor da suspensão.

O preenchimento dos campos da janela Create Damper se dá conforme mostrado na Figura 5.85. O amortecedor é definido entre os corpos rígidos (partes) "Upper_strut" e "Lower_strut" e sua curva característica pode ser inserida do próprio software ou editada manualmente pelo usuário e utilizada no modelo. Como se pode observar na Figura 5.85, no campo Property File, as propriedades do amortecedor são definidas no arquivo especificado na biblioteca do software.

- $\quad$ Clique em $O K$.

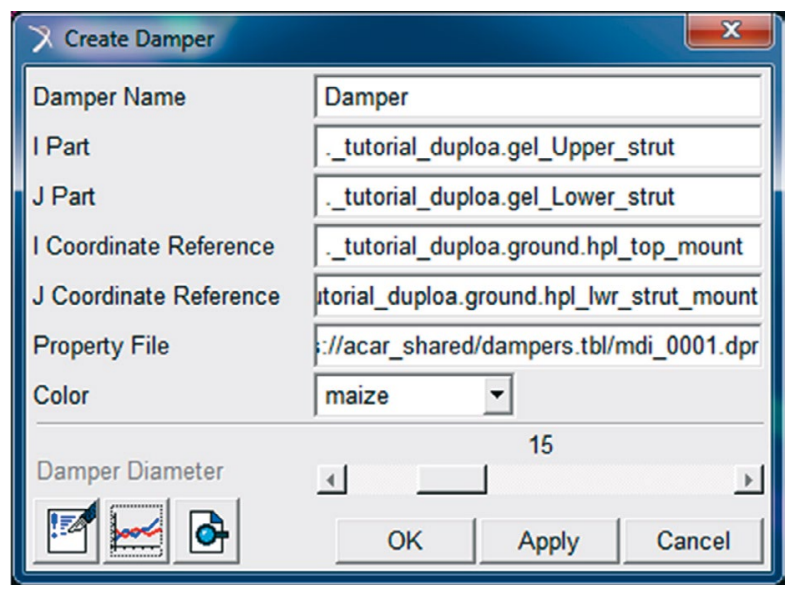

Figura 5.85 - Definição do amortecedor da suspensão.

A Figura 5.86 ilustra o amortecedor construído. 


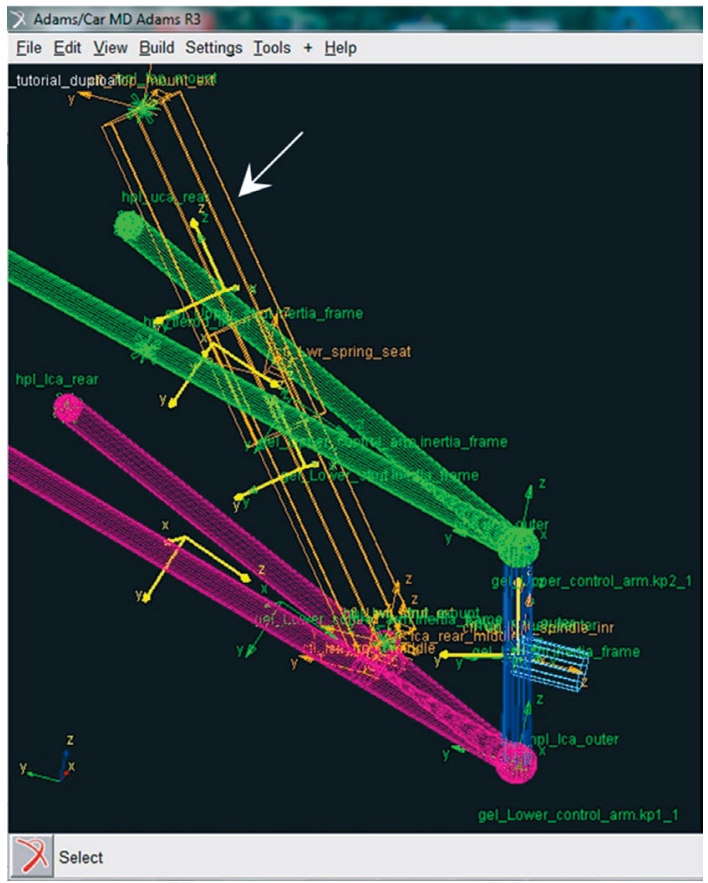

Figura 5.86 - Tela ilustrativa do amortecedor construído.

- $\quad$ Salve seu projeto.

Etapa 15 - Construção da mola (Spring)

Nesta etapa, será definida a mola da suspensão. Ressalta-se que a mola não é considerada um corpo rígido, portanto sua criação não será por meio da criação de um General Part. No ADAMS/Car, a mola é definida por meio da criação de uma força (Forces). Entretanto, o processo de construção da mola necessita, antes, da criação de um Mount, que pode ser criado acessando-se o menu Build $\rightarrow$ Parts $\rightarrow$ Mount $\rightarrow$ New (Figura 5.87). 


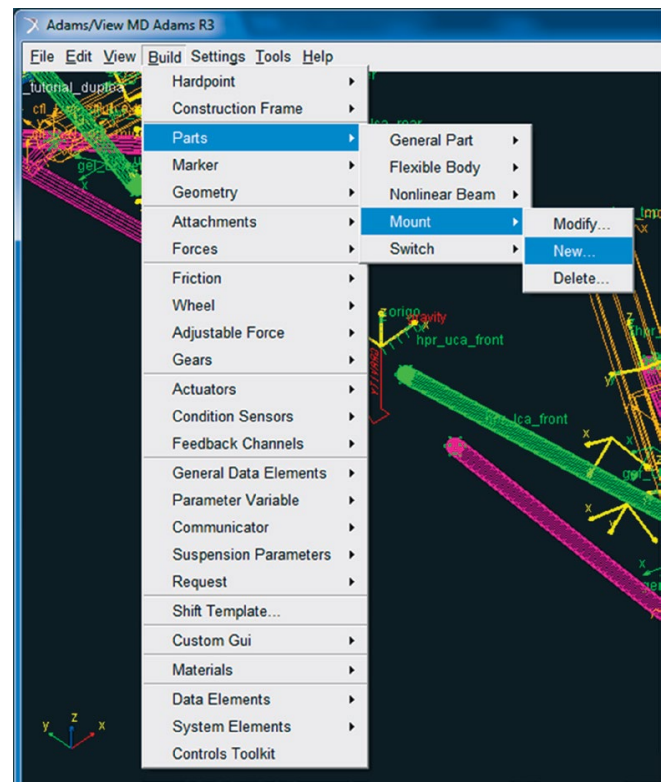

Figura 5.87 - Criaçãodo Mount para construção da mola.

A janela Create Mount Part será aberta, e os campos a serem preenchidos estão ilustrados na Figura 5.88.

- $\quad$ Clique em $O K$.

\begin{tabular}{|c|c|c|c|}
\hline \multicolumn{3}{|l|}{$X$ Create Mount Part } & $x$ \\
\hline Mount Name & \multicolumn{3}{|c|}{ strut_to_body } \\
\hline Coordinate Reference & \multicolumn{3}{|c|}{ |_tutorial_duploa.ground.hpl_top_mount } \\
\hline From Minor Role & inherit & - & \\
\hline FEd & OK & Apply & Cancel \\
\hline
\end{tabular}

Figura 5.88 - Janela de criação do Mount para construção da mola.

A Figura 5.89 ilustra o Mount criado. 


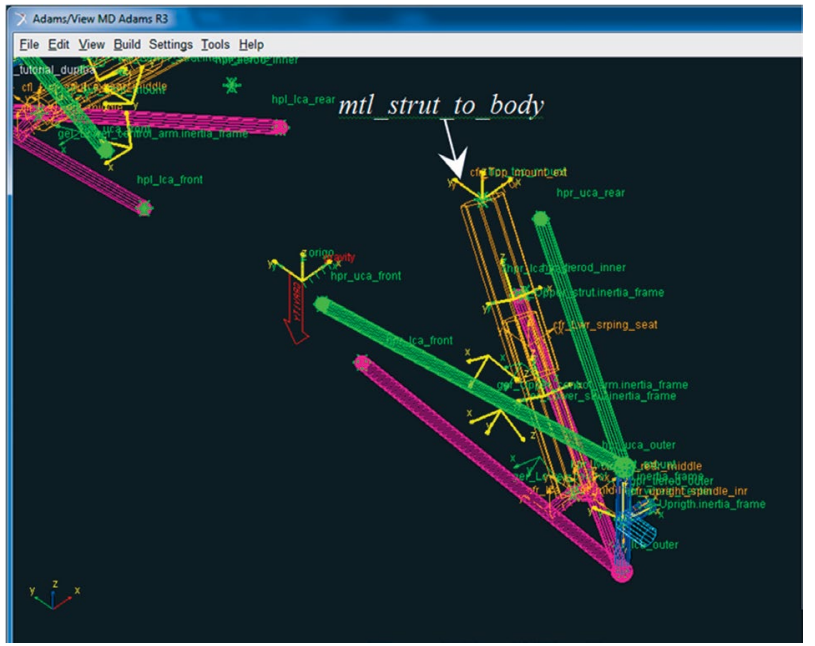

Figura 5.89-Tela ilustrativa do Mount para a construção da mola

- Com o Mount construído, é possível, então, criar a mola. Para isso, acesse o menu Build $\rightarrow$ Forces $\rightarrow$ Spring $\rightarrow$ New (Figura 5.90).

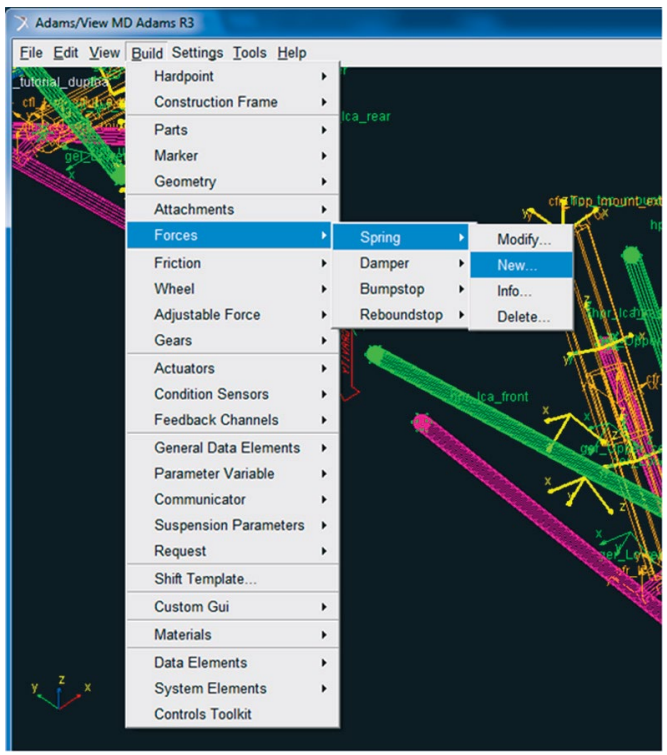

Figura 5.90 - Janela de opção para criação de uma mola.

- Preencha os campos da janela Create Spring conforme mostrado na Figura 5.91. Note que a mola é definida entre o corpo rígido Lower_control_arm e mount que se criou nessa etapa $m t l \_s t r u t \_t o \_b o d y$. A curva característica da mola pode ser editada manualmente pelo usuário e salva 
em arquivo externo ou podem ser utilizadas informações da própria biblioteca do software (campo Property File) como é o caso deste modelo. - $\quad$ Clique em $O K$.

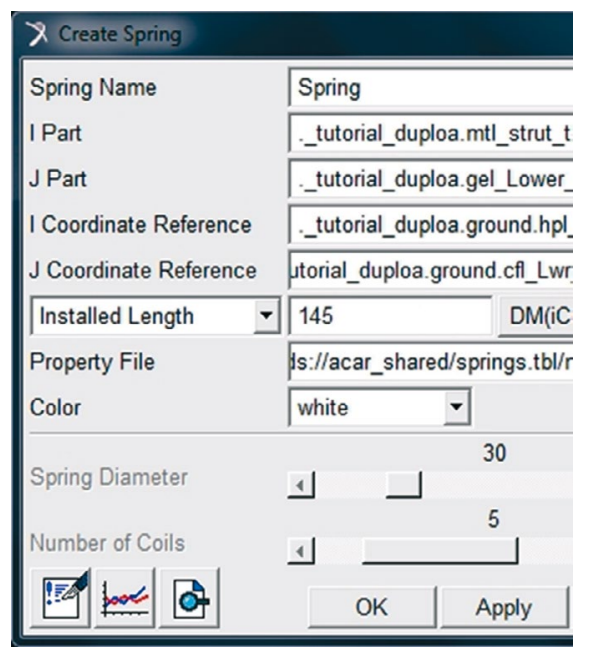

Figura 5.91 - Janela correspondente à definição da mola da suspensão.

A Figura 5.92 ilustra a mola construída.

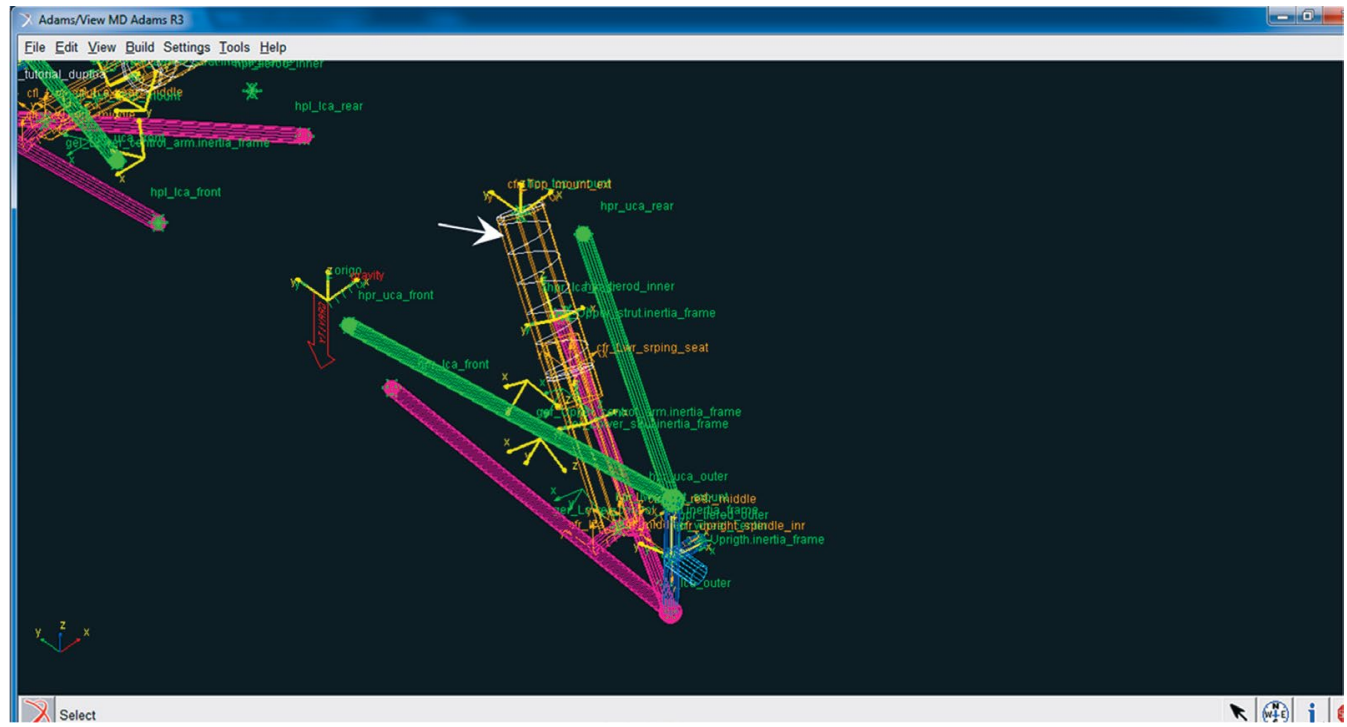

Figura 5.92 - Mola construída. 
A notar: BumpStop ou batente de suspensão tem a função de limitar o funcionamento do conjunto mola e amortecedor, evitando deslocamentos que possam prejudicar o funcionamento do conjunto.

- É necessário criar o Bumpstop da suspensão para limitar o seu deslocamento. Para isso, acesse o menu Build $\rightarrow$ Forces $\rightarrow$ Bumpstop $\rightarrow$ New (Figura 5.93).

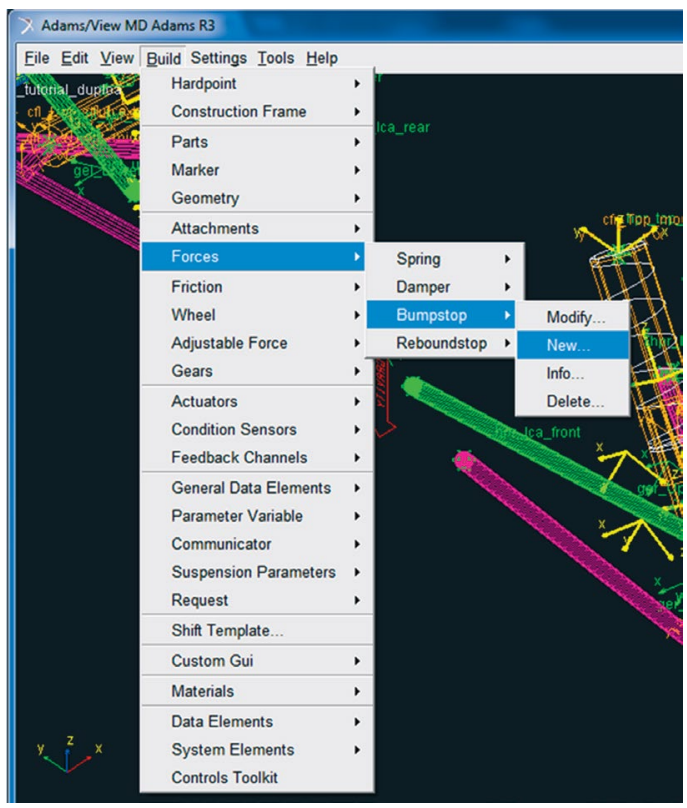

Figura 5.93 - Criando Bumpstop.

- $\quad$ Preencha os campos da janela Create Bumpstop conforme mostrado na Figura 5.94. A curva característica do Bumpstop pode ser editada manualmente pelo usuário e salvo em arquivo externo ou podem ser utilizadas informações da própria biblioteca do software (campo Property File) como é o caso deste modelo.

- $\quad$ Clique em $O K$. 


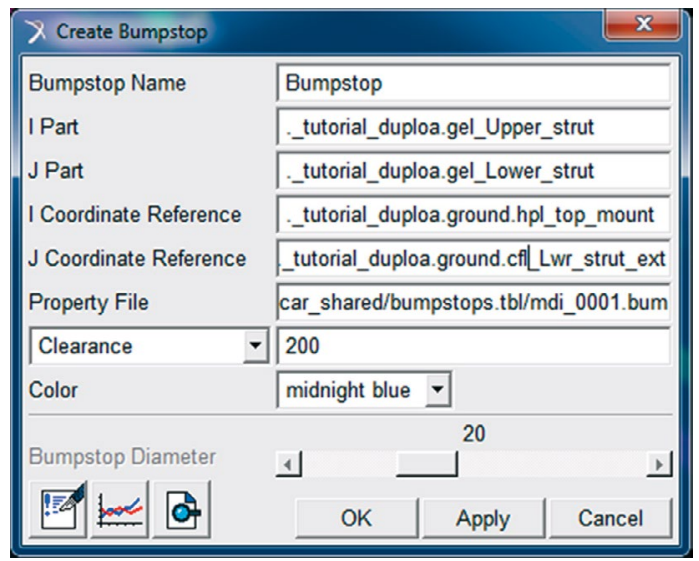

Figura 5.94 - Janela de definição das características do Bumpstop.

\section{A Figura 5.95 ilustra o Bumpstop construído.}

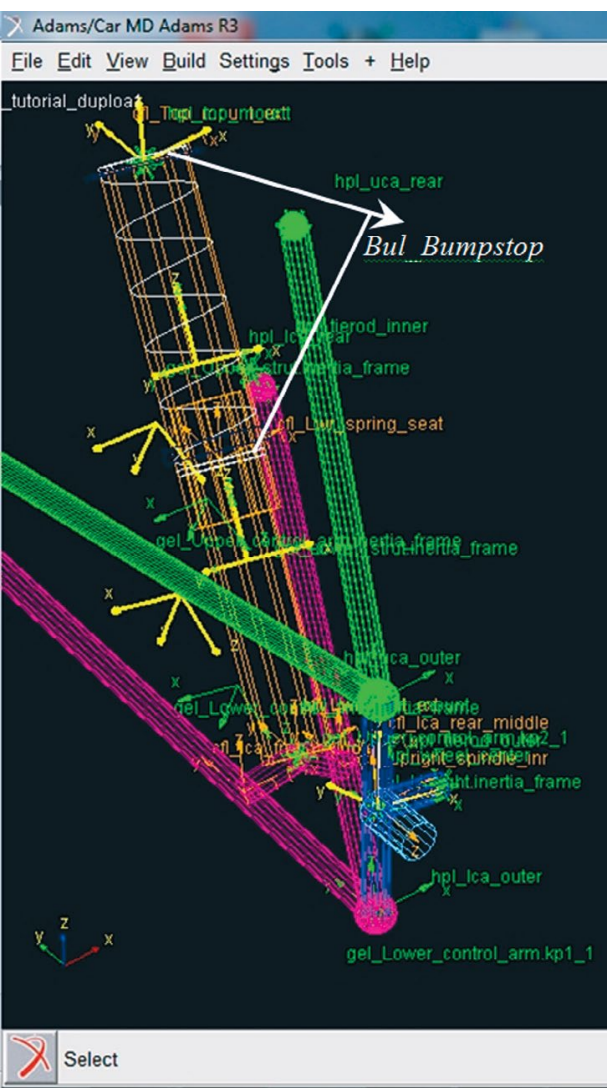

Figura 5.95 - Tela ilustrativa do Bumpstop criado nas extremidades da mola.

- $\quad$ Salve seu projeto. 


\section{Etapa 16 - Construção da barra de direção (Tierod)}

Nesta etapa, será construída a barra de direção, a qual será um General Part denominado por Tierod.

- $\quad$ Acesse o menu Build $\rightarrow$ Parts $\rightarrow$ General Part $\rightarrow$ New (Figura 5.96).

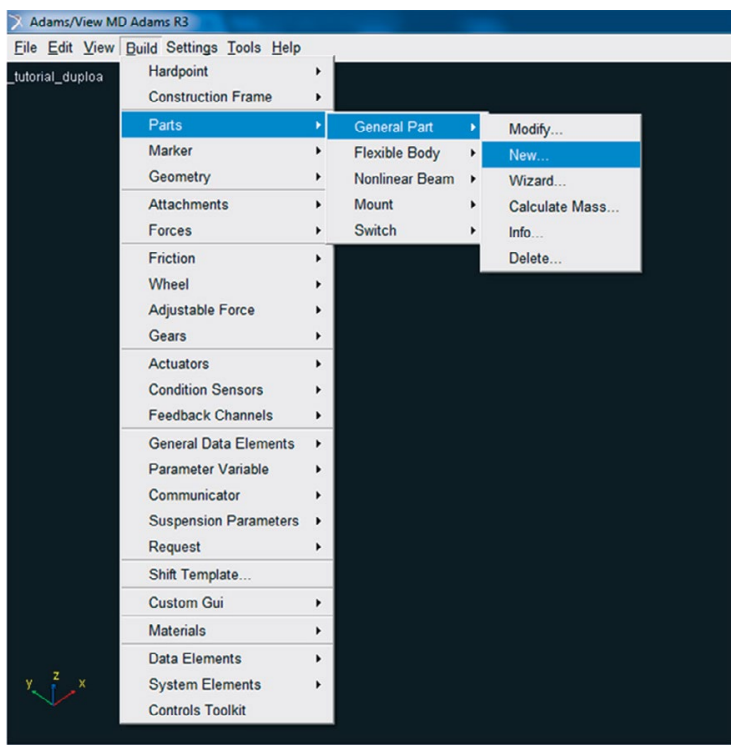

Figura 5.96 - Janela correspondente à criação do General Part "Tierod".

- Preencha os campos da janela Create General Part, conforme ilustrado na Figura 5.97.

Note que a geometria (Geometry) associada a este General Part será do tipo Link, sendo este último definido pelos Hardpoint "hpl_tierod_inner" e “hpl_tierod_outer".

- $\quad$ Clique em $O K$. 


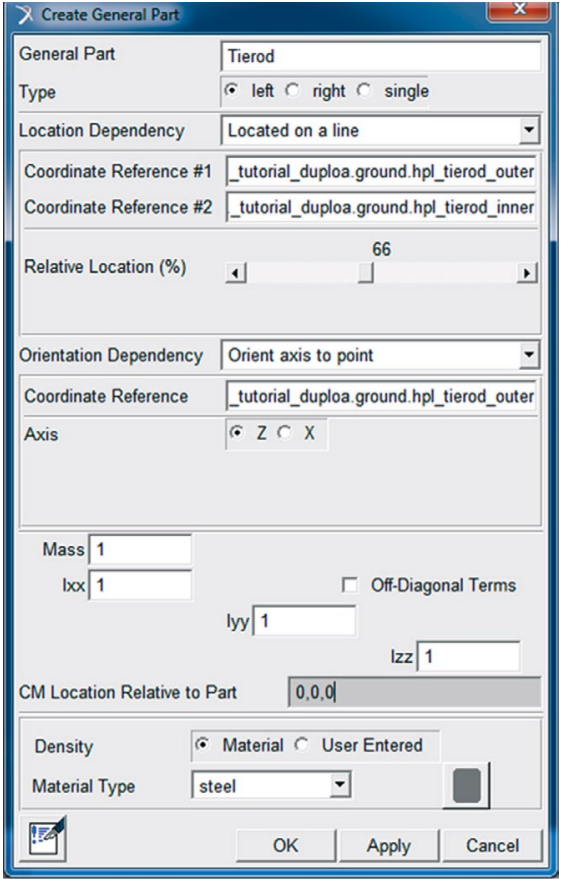

Figura 5.97 - Preenchimento da caixa de diálogo para construção do General Part "Tierod".

A Figura 5.98 ilustra como a suspensão deve estar até o presente momento.

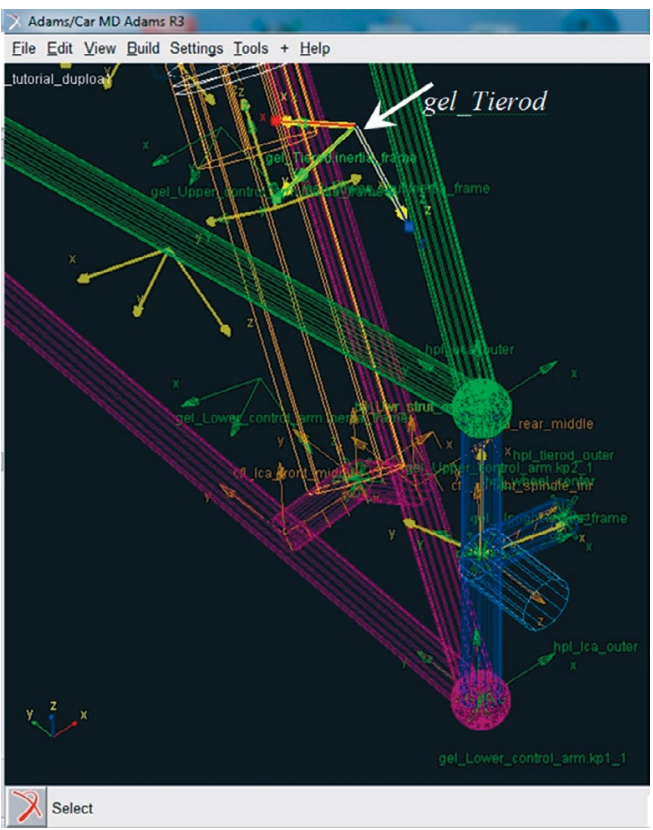

Figura 5.98 - Ilustração do General Part "Tierod" construído. 
- Para criação do Link referente ao braço de direção, acesse o menu Build $\rightarrow$ Geometry $\rightarrow$ Link $\rightarrow$ New (Figura 5.99).

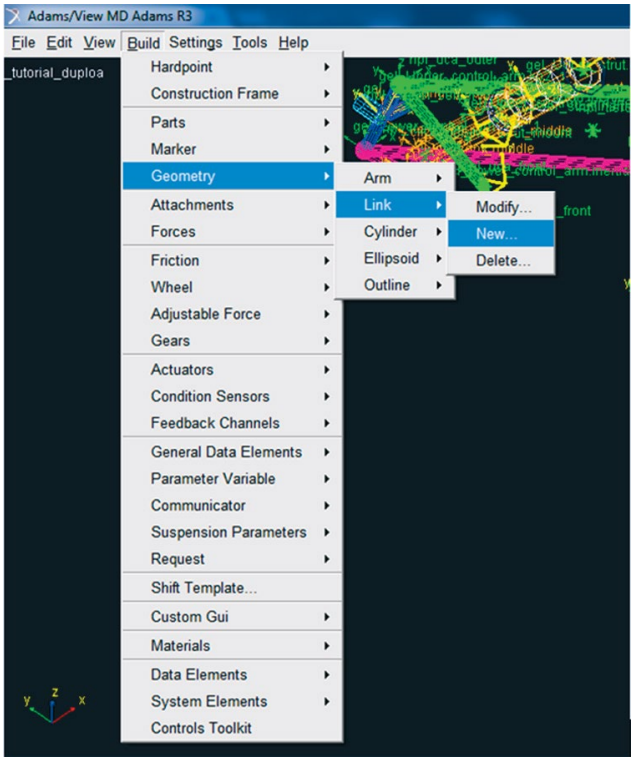

Figura 5.99 - Janela correspondente à criação da geometria referente General Part "Tierod".

- Preencha os campos da janela Create Link Geometry, conforme mostrado na Figura 5.100.

\begin{tabular}{|c|c|}
\hline \multicolumn{2}{|l|}{ Create Link Geometry } \\
\hline Link Name & Link10 \\
\hline General Part & _tutorial_duploa.gel_Tierod \\
\hline Coordinate Reference \#1 & _tutorial_duploa.ground.hpl_tierod_outer \\
\hline Coordinate Reference \#2 & _tutorial_duploa.ground.hpl_tierod_inner \\
\hline Radius & 10 \\
\hline Color & white \\
\hline \multicolumn{2}{|c|}{ Iv Calculate Mass Properties of General Part } \\
\hline Density & (. Material $\subset$ User Entered \\
\hline Material Type & steel $\quad=$ \\
\hline EEA & Cancel \\
\hline
\end{tabular}

Figura 5.100 - Janela de criação do link referente ao braço de direção.

- $\quad$ Clique em $O K$. 
A Figura 5.101 ilustra o braço de direção criado a partir das informações da Figura 5.100.

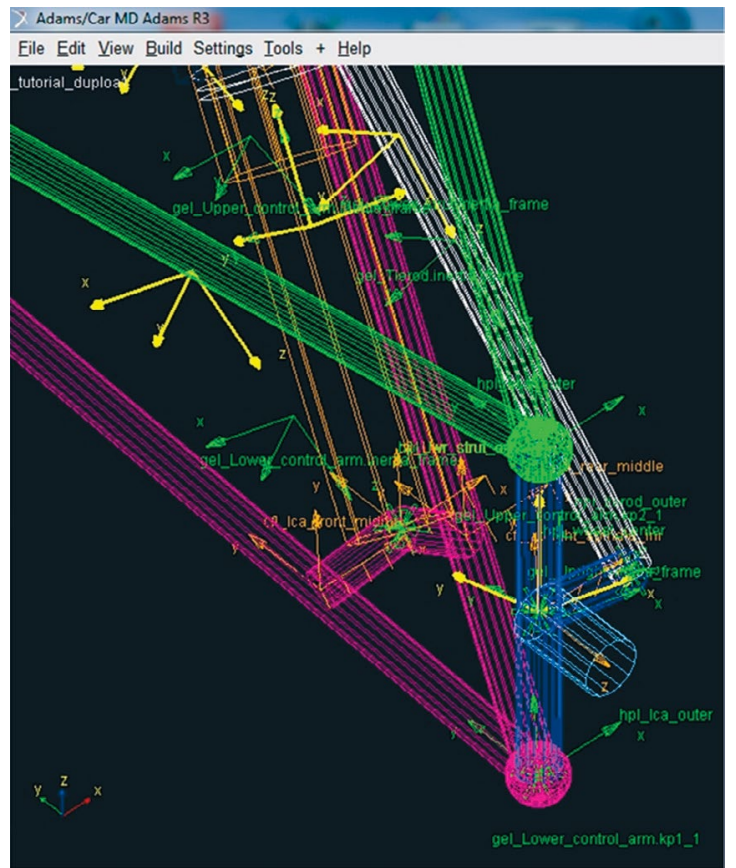

Figura 5.101 - Ilustração do Link representando a barra de direção (em branco).

- Em seguida, crie esferas nas extremidades do Link10 (Figura 5.100). Para a criação dessas esferas, acesse o menu Build $\rightarrow$ Geometry $\rightarrow$ Ellipsoid $\rightarrow$ New (Figura 5.102). 


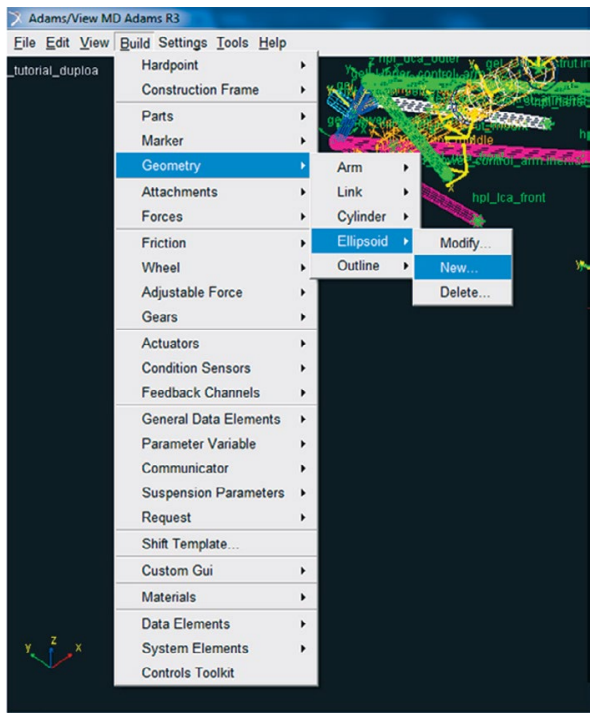

Figura 5.102 - Janela correspondente à criação de esferas utilizando a opção Ellipsoid.

- A janela Create Ellipsoid Geometry será aberta. Os campos deverão ser preenchidos conforme Figura 5.103. Lembre-se que o campo Calculate Mass Properties of General Part deverá estar selecionado para atualização das propriedades de massa do General Part "Tierod".

- Clique em Apply.

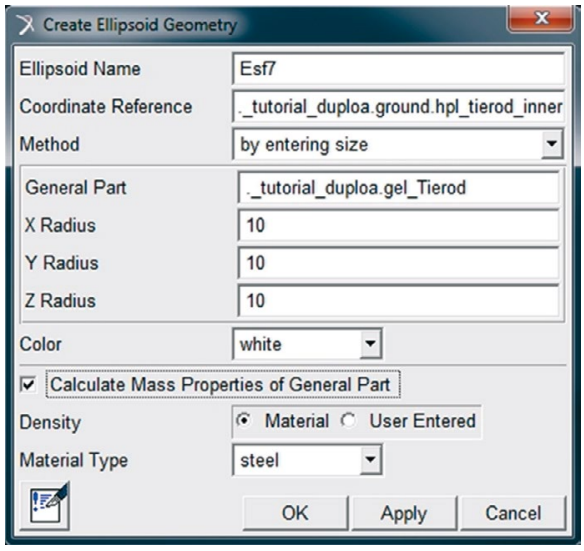

Figura 5.103 - Janela relativa à criação da esfera do Link10.

- Repita o procedimento descrito acima para a construção de outra esfera a se situar no Hardpoint hpl_tierod_outer. O preenchimento da janela Create Ellipsoid Geometry para essa geometria está ilustrado na Figura 5.104. 


\begin{tabular}{|c|c|c|}
\hline \multicolumn{2}{|c|}{ X Create Ellipsoid Geometry } & $x$ \\
\hline \multirow{3}{*}{$\begin{array}{l}\text { Ellipsoid Name } \\
\text { Coordinate Reference } \\
\text { Method }\end{array}$} & \multirow{2}{*}{\multicolumn{2}{|c|}{\begin{tabular}{|l} 
Esf8 \\
-_tutorial_duploa.ground.hpl_tierod_outer
\end{tabular}}} \\
\hline & & \\
\hline & by entering size & 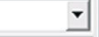 \\
\hline General Part & \multicolumn{2}{|l|}{ _._tutorial_duploa.gel_Tierod } \\
\hline X Radius & \multicolumn{2}{|l|}{10} \\
\hline Y Radius & \multicolumn{2}{|l|}{10} \\
\hline Z Radius & \multicolumn{2}{|l|}{10} \\
\hline Color & white & \\
\hline \multicolumn{3}{|c|}{ IV Calculate Mass Properties of General Part } \\
\hline Density & \multicolumn{2}{|l|}{ C Material $C$ User Entered } \\
\hline Material Type & \multicolumn{2}{|l|}{\begin{tabular}{|l|l} 
steel & $\quad$
\end{tabular}} \\
\hline Fed & Apply & Cancel \\
\hline
\end{tabular}

Figura 5.104 - Preenchimento da janela de criação da esfera Esf8.

- $\quad$ Clique em OK.

A Figura 5.105 ilustra as esferas criadas nas extremidades da barra de direção, segundo as Figuras 5.103 e 5.104 .

- Salve seu projeto.

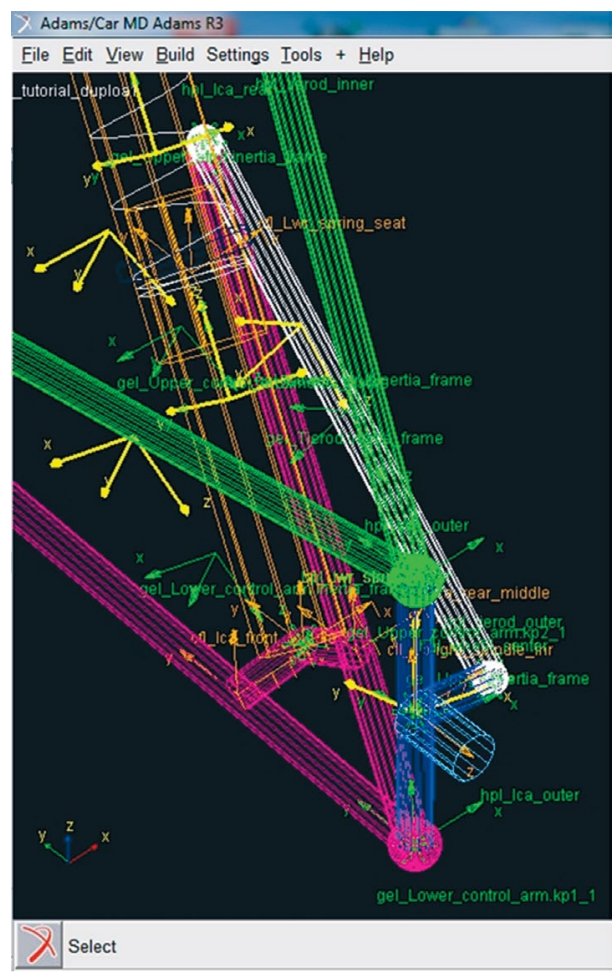

Figura 5.105 - Ilustração das esferas criadas nas extremidades da barra de direção (em branco). 


\section{Etapa 17 - Criação dos Mounts de conexão da suspensão}

Nesta etapa, serão criados três Mounts denominados uca_to_body, subframe_to_body e tierod_to_steering, os quais são classificados como Parts, de acordo com o ADAMS/Car.

Esses Mounts possuem a função de estabelecer a conexão (compatibilidade de informação) de um subsistema com outro, no caso a suspensão com o chassi (body) e o sistema de direção. Durante a simulação, a transferência de dados de um subsistema para o outro é feita por meio desta comunicação (Mount). Para criar um Mount:

- $\quad$ Acesse o menu Build $\rightarrow$ Parts $\rightarrow$ Mount $\rightarrow$ New (Figura 5.106).

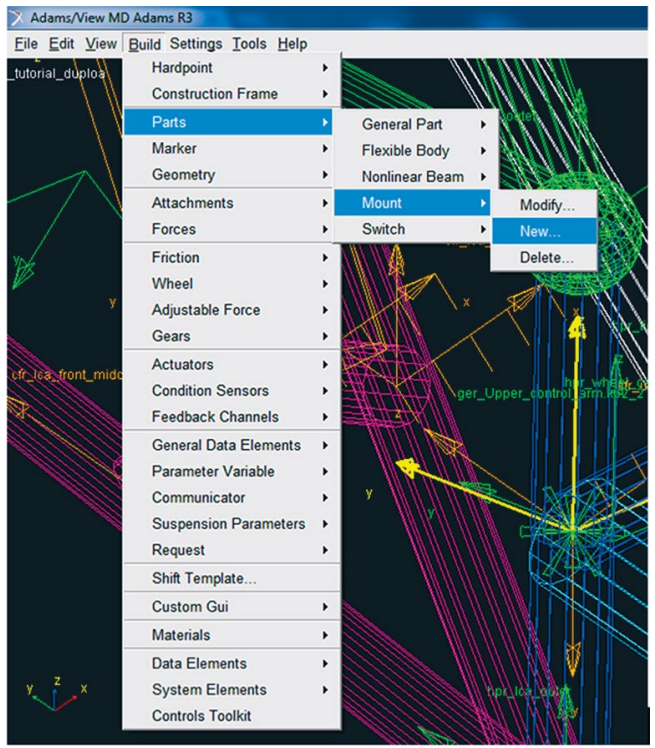

Figura 5.106 - Ilustração do menu/opções correspondente à criação do Mount "uca_to_body".

- A janela Create Mount Part deverá ter seus campos preenchidos, conforme mostrado na Figura 5.107.

Note que o nome do Mount deve ser exatamente o mesmo definido no outro susbsistema, nesse caso, o chassi, ou seja, no chassi deve haver um Mount de mesma denominação.

Ainda com relação a Figura 5.107, a coordenada de referência utilizada é o Hardpoint "bpl_uca_front".

- Ao finalizar a operação clique em Apply. 


\begin{tabular}{|c|c|c|c|}
\hline \multicolumn{3}{|l|}{$\chi$ Create Mount Part } & $x$ \\
\hline Mount Name & \multicolumn{3}{|c|}{ uca_to_body } \\
\hline Coordinate Reference & \multicolumn{3}{|c|}{-_tutorial_duploa.ground.hpl_uca_front } \\
\hline From Minor Role & inherit & 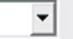 & \\
\hline$E$ & OK & Apply & Cancel \\
\hline
\end{tabular}

Figura 5.107 - Janela correspondente à criação do Mount "uca_to_ body”.

Na Figura 5.108 o Mount “uca_to_body" está destacado com uma seta branca.

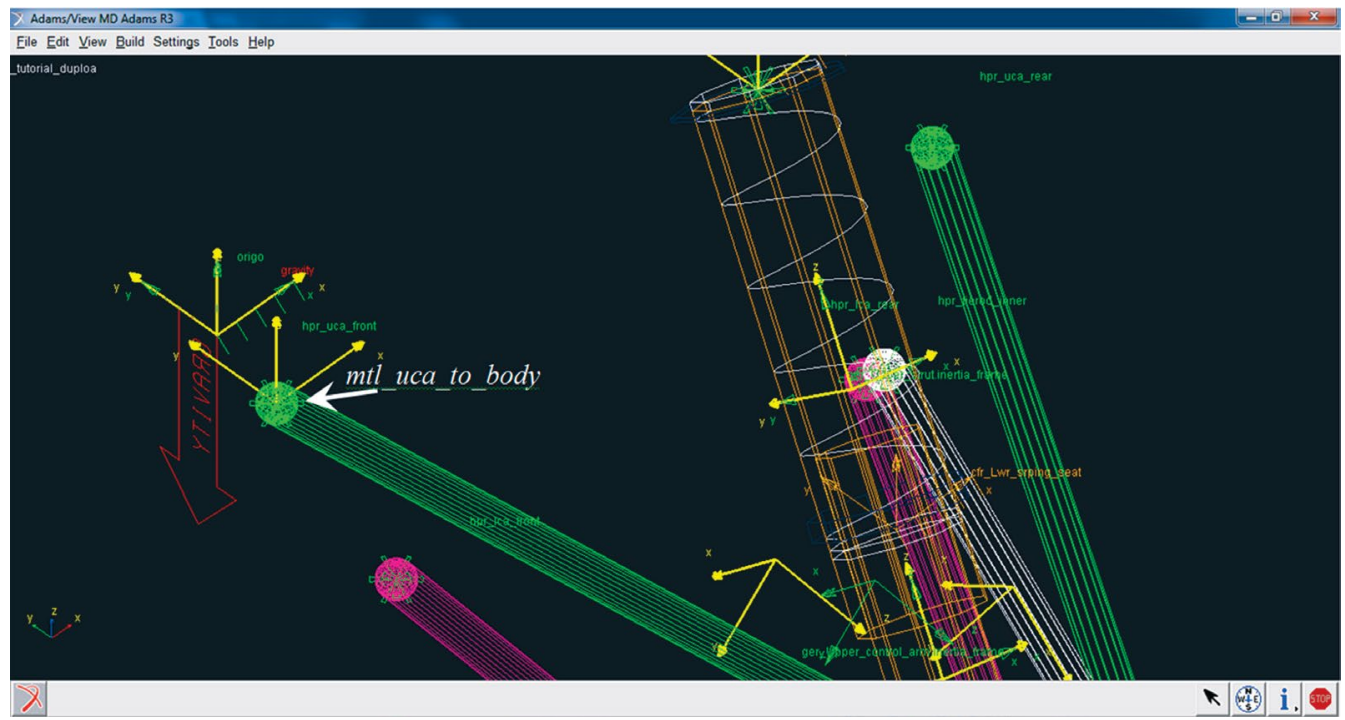

Figura 5.108 - Ilustração do Mount “uca_to_body” criado.

- Repita o procedimento anterior para criação dos dois outros Mounts, "subframte_to_body" e "tierod_to_steering”. Para isso:

- Utilize o preenchimento apresentado na Figura 5.109 para o Mount “subframe_to_body" e o preenchimento dado na Figura 5.110 para o Mount "tierod_to_steering".

\begin{tabular}{|l|l|}
\hline X Create Mount Part & \\
\hline Mount Name & subframe_to_body \\
Coordinate Reference & .tutorial_duploa.ground.hpl_Ica_front \\
From Minor Role & inherit \\
\hline
\end{tabular}

Figura 5.109 - Preenchimento da caixa de diálogo relativa à criação do Mount "subframe_to_body". 
- Ao finalizar o preenchimento da caixa de diálogo, ilustrada na Figura 5.109, clique em Apply.

\begin{tabular}{|c|c|c|c|}
\hline$\chi$ Create Mount Part & & & $x$ \\
\hline Mount Name & \multicolumn{3}{|c|}{ tierod_to_steering } \\
\hline Coordinate Reference & \multicolumn{3}{|c|}{ _tutorial_duploa.ground.hpl_tierod_inner } \\
\hline From Minor Role & inherit & $\nabla$ & \\
\hline EDd & OK & Apply & Cancel \\
\hline
\end{tabular}

Figura 5.110 - Preenchimento da caixa de diálogo relativa à criação do Mount "tierod_to_steering".

- Ao finalizar o preenchimento da caixa de diálogo, ilustrada na Figura 5.110, clique em $O K$.

$\mathrm{Na}$ Figura 5.111 é mostrado como os Mounts devem estar localizados no subsistema de suspensão.

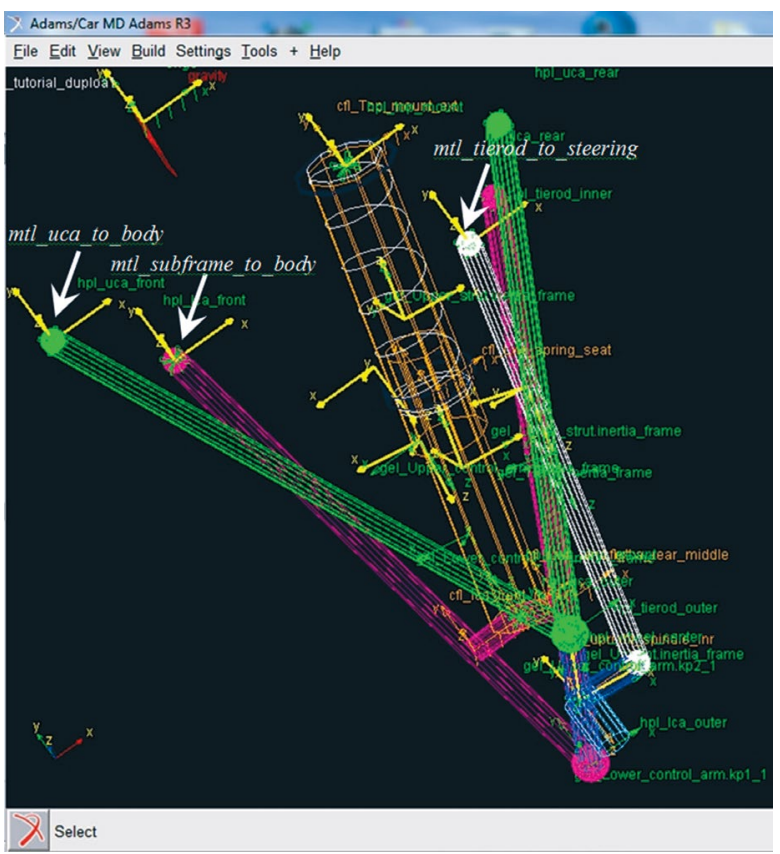

Figura 5.111 - Ilustração dos Mounts "subframe_to_body" e "tierod_to_steering" criados.

- $\quad$ Salve seu projeto.

Etapa 18 - Criação das juntas de conexão da suspensão

Nesta etapa, serão inseridas quatro juntas no modelo virtual da suspensão. Essas juntas definem o tipo de movimento (graus de liberdade e restrições) entre os General Part. A Tabela 5.2 relaciona as juntas a serem criadas. 


\section{Tabela 5.2 - Juntas do modelo multicorpos da suspensão}

\begin{tabular}{c|c|c} 
Junta & Tipo & General Parts \\
\hline 1 & Esférica & Uprigth e Lower_control_arm \\
\hline 2 & Esférica & Uprigth e Upper_control_arm \\
\hline 3 & Esférica & Uprighte Tierod \\
\hline 4 & Revoluta & Spindle e Upright \\
\hline
\end{tabular}

Para inserção das juntas da suspensão:

- Acesse o menu Build $\rightarrow$ Attachments $\rightarrow$ Joints $\rightarrow$ New (Figura 5.112).

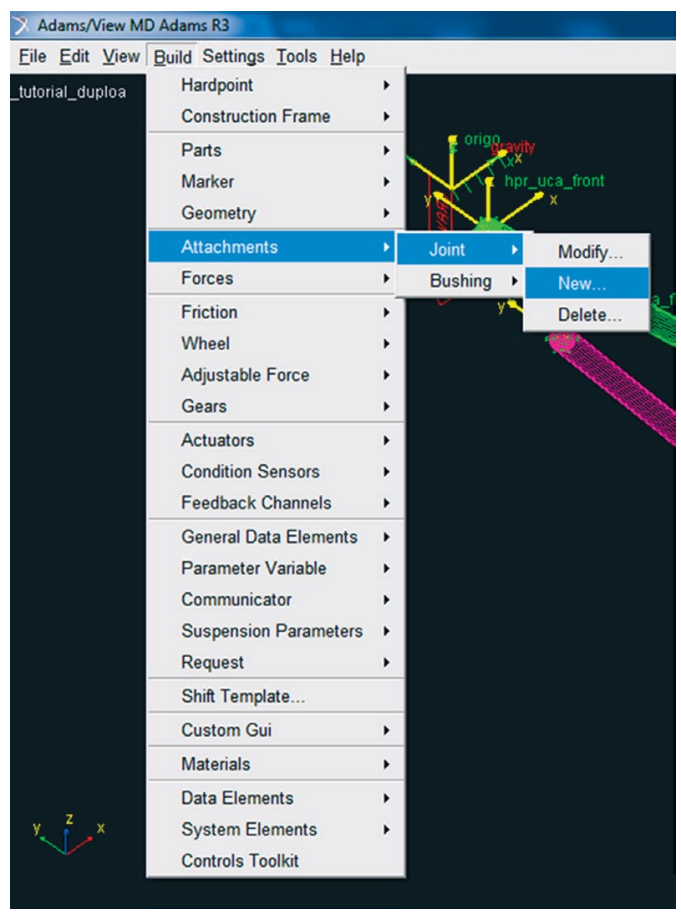

Figura 5.112 - Janela correspondente à criação de uma Junta, utilizando a opção Joint.

Os campos da janela Create Joint Attachment deverão ser preenchidos, conforme ilustrado na Figura 5.113 e de acordo com a Tabela 5.2.

- Após finalizar o preenchimento sugerido na Figura 5.113, clique em Apply. 
- Em seguida, defina a junta 2 (Tabela 5.2), preenchendo a janela Create Joint Attachment, mostrada na Figura 5.115.

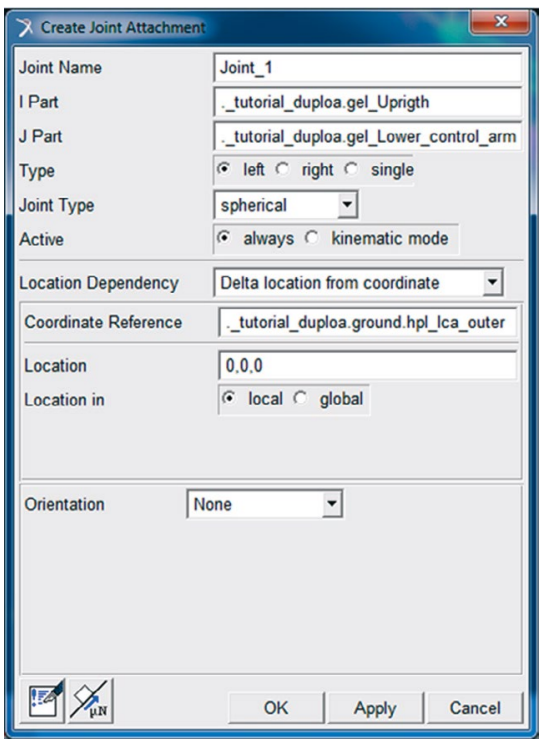

Figura 5.113 - Preenchimento da caixa de diálogo para criação da Junta 1.

A Figura 5.114 ilustra a junta 1 construída.

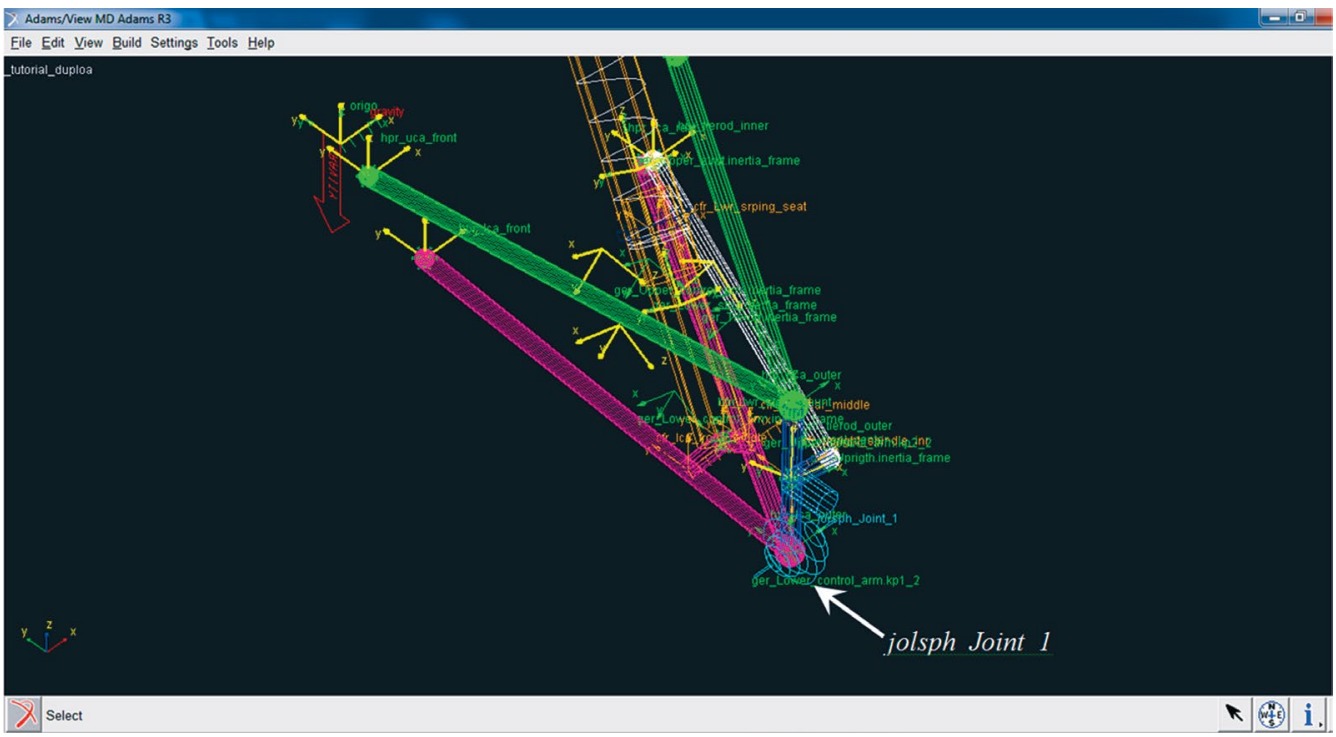

Figura 5.114 - Ilustração da junta 1 construída. 
A notar: Perceba que a nomenclatura de juntas no ADAMS/Car segue a seguinte denominação; jol para especificação que o tipo de Attachment utilizado é uma junta à esquerda seguida da especificação do tipo, no caso da Junta_1 trata-se de uma junta esférica (spherical).

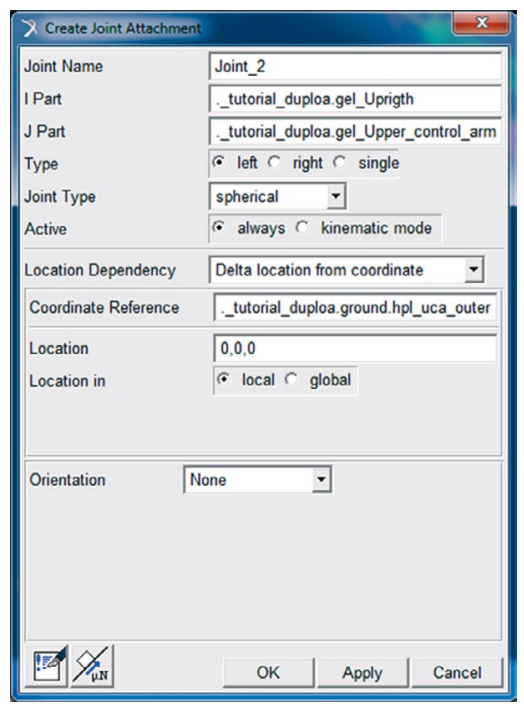

Figura 5.115 - Preenchimento da caixa de diálogo para criação da Junta 2.

A Figura 5.116 ilustra a junta 2 construída.

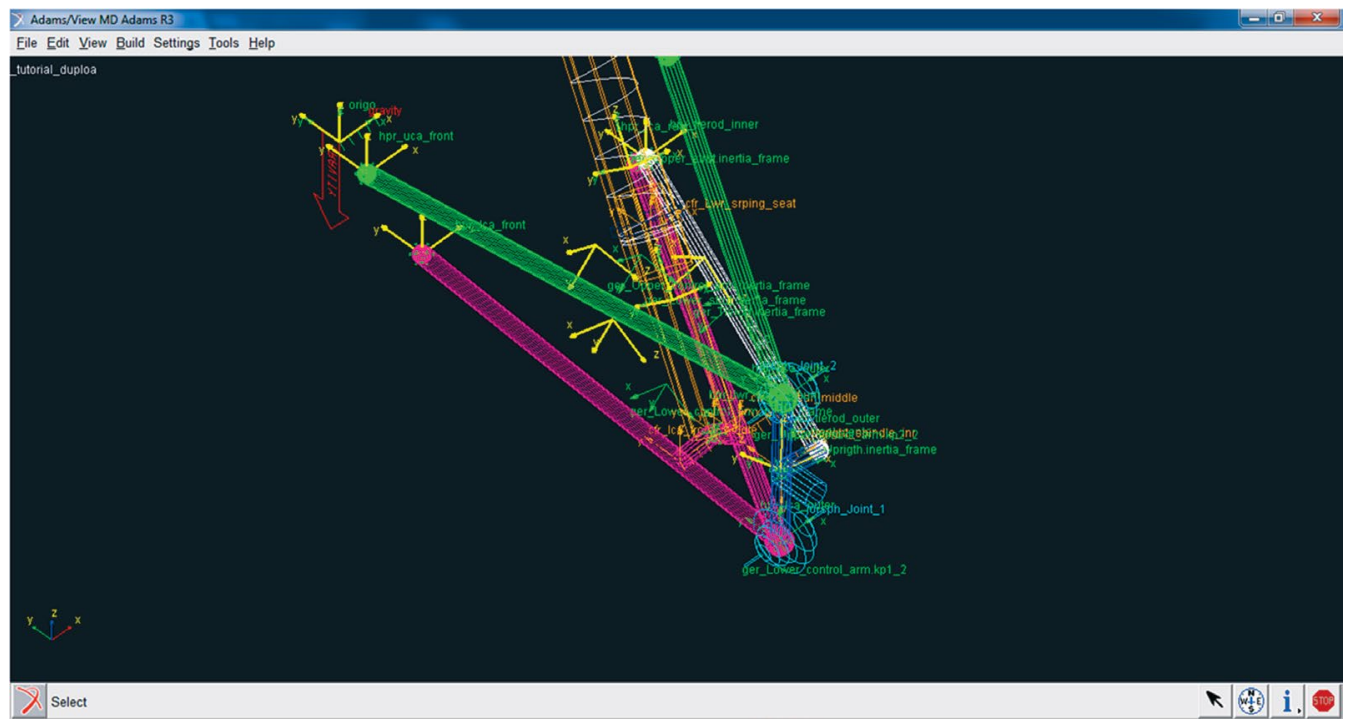

Figura 5.116 - Ilustração da junta 2 construída. 
Para construção da junta 3, proceda como sugerido a seguir:

- Preencha os campos da janela Create Joint Attachment, conforme a Figura 5.117.

- $\quad$ Clique em $O K$.

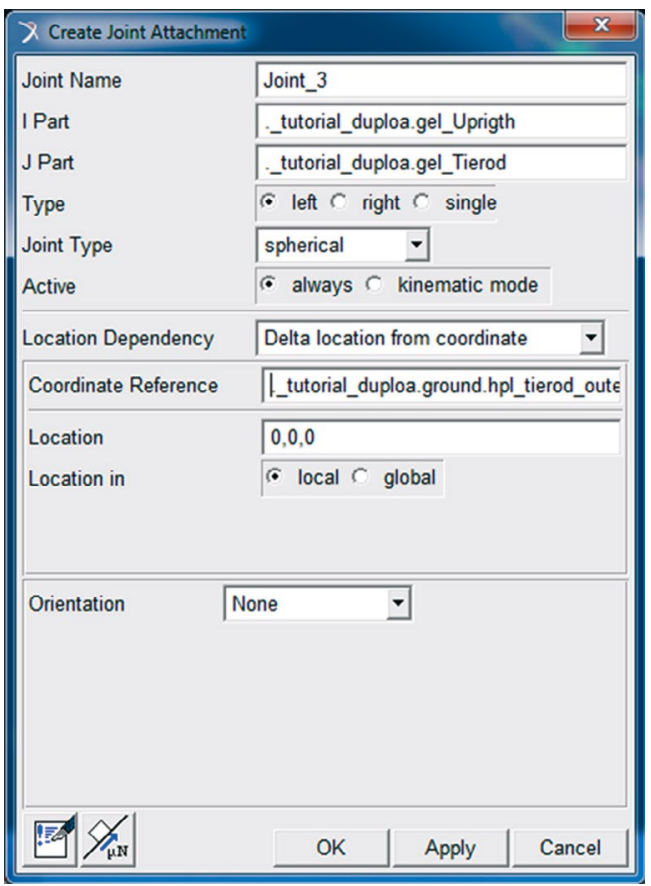

Figura 5.117 - Preenchimento da caixa de diálogo para criação da Junta 3.

A Figura 5.118 ilustra a junta 3 construída. 


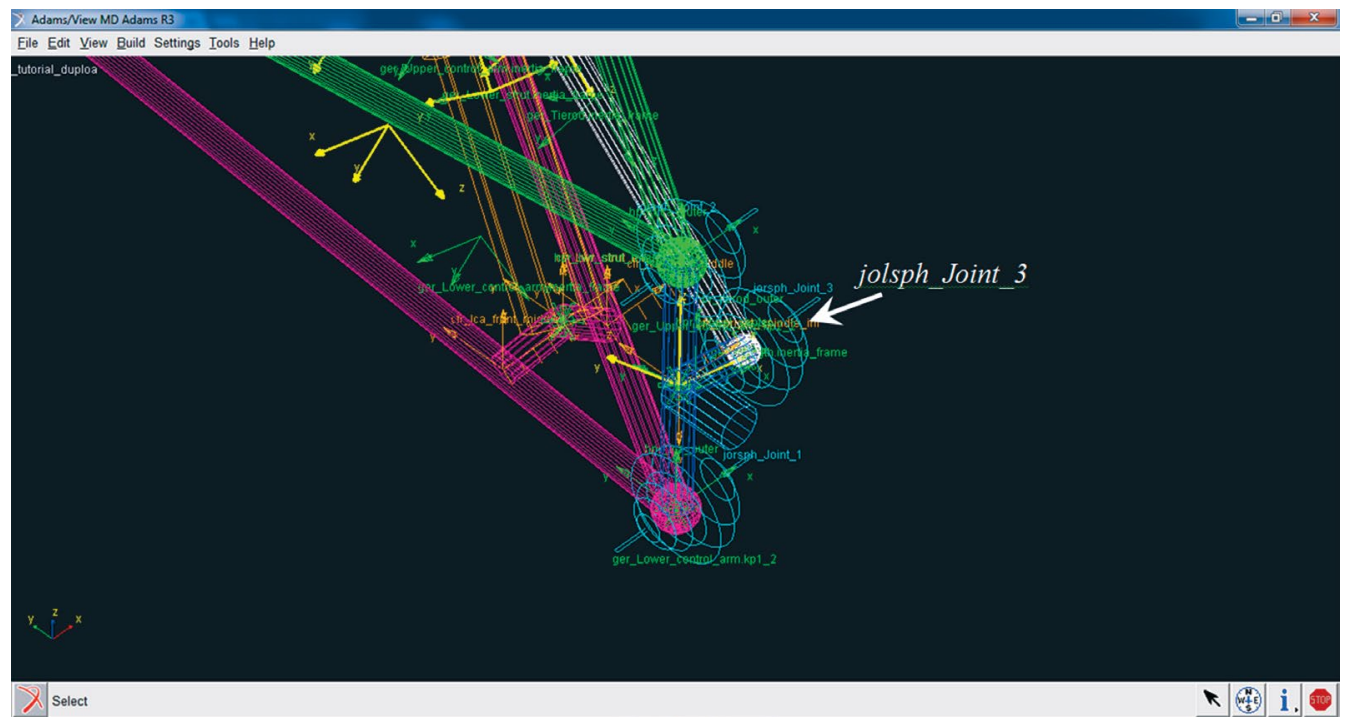

Figura 5.118 - Ilustração da junta 3 construída.

Neste ponto, antes da construção da próxima junta (Joint_4), que será uma junta revoluta (Tabela 5.2), deve-se proceder a construção de um General Part denominado Spindle. Perceba que, tanto sua localização (Location Dependancy), quanto sua orientação (Orientation Dependancy) são dependentes do Construction Frame (cfl) "Upright_spindle_inr”, Figura 5.79 e Figura5.80. As informações para construção do General Part deve seguir o especificado na Figura 5.119 (a).

- Acesse Build $\rightarrow$ Parts $\rightarrow$ General Part $\rightarrow$ New.

- Após preenchimento, clique em $O K$.

A Figura 5.119 (b) ilustra a criação do General Part "Spindle". 


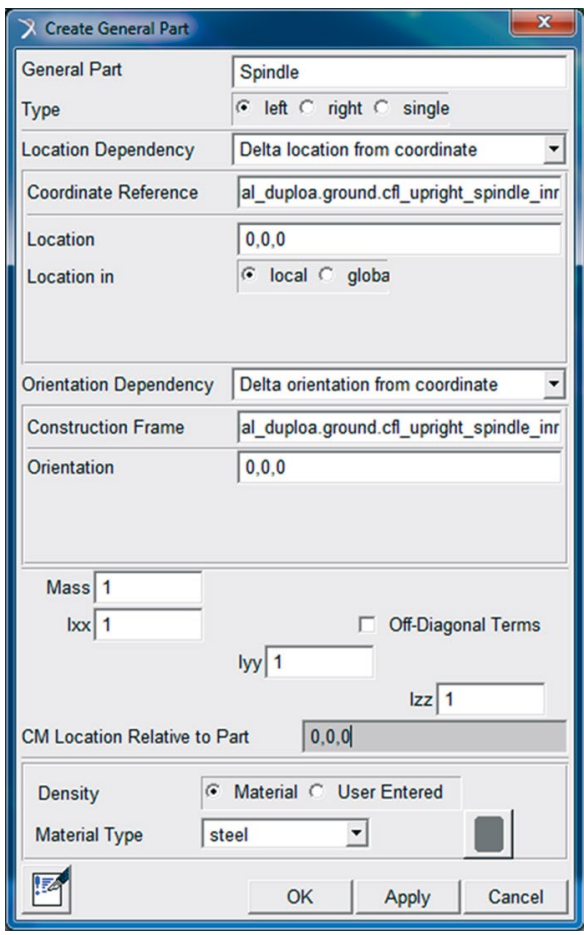

Figura 5.119 (a) - Preenchimento da caixa de diálogo para criação do General Part "Spindle".

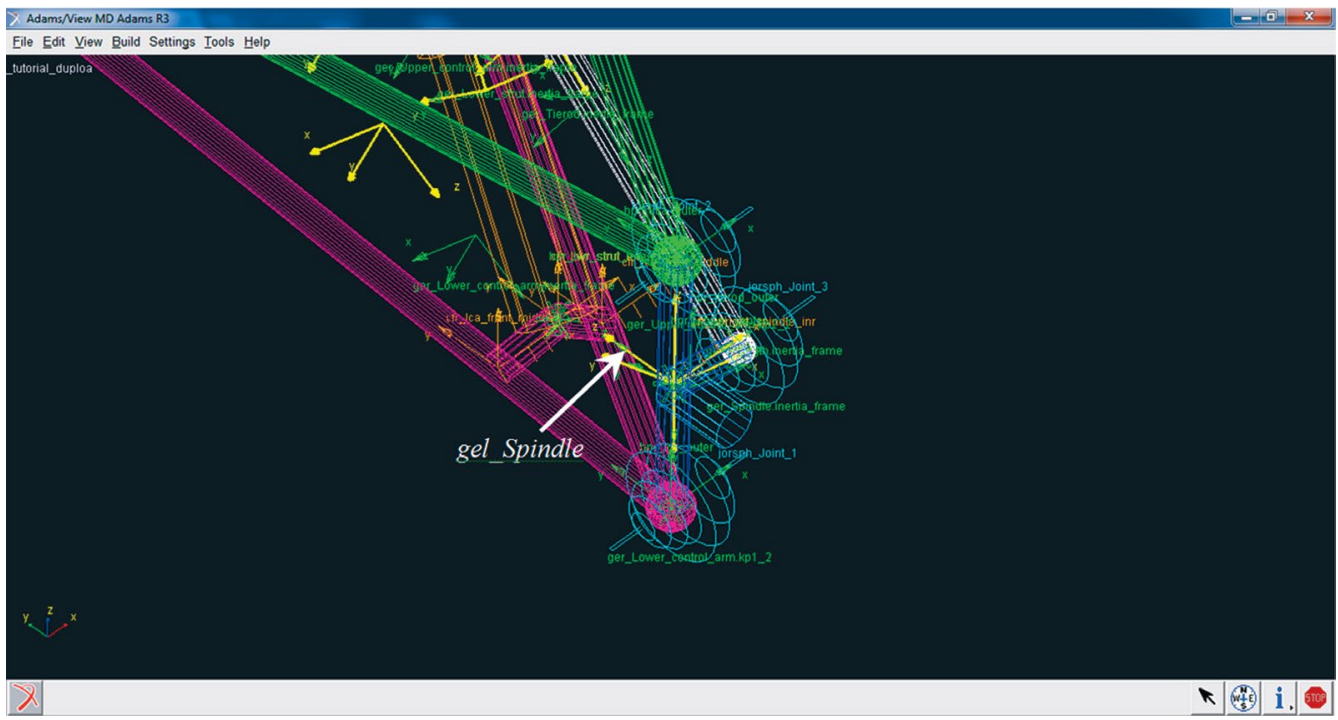

Figura 5.119 (b) - General Part "Spindle” construído. 
Em seguida, parte-se para a construção da quarta junta, que é revoluta (Tabela 5.2). Para tanto, siga as instruções abaixo:

- Acesse a opção Build $\rightarrow$ Attachments $\rightarrow$ Joints $\rightarrow$ New.

- Preencha os campos da janela Create Joint Attachment, conforme a Figura 5.120.

- $\quad$ Clique em $O K$.

- Salve seu projeto.

A Figura 5.121 traz a tela resultante, referente a este passo.

\begin{tabular}{|c|c|c|}
\hline \multicolumn{2}{|l|}{ C Create Joint Attachment } & $x$ \\
\hline Joint Name & \multicolumn{2}{|l|}{ Joint_4 } \\
\hline I Part & \multicolumn{2}{|l|}{-_tutorial_duploa.gel_Spindle } \\
\hline J Part & \multicolumn{2}{|l|}{._tutorial_duploa.gel_Uprigth } \\
\hline Type & \multicolumn{2}{|l|}{ - left $C$ right $C$ single } \\
\hline Joint Type & \multicolumn{2}{|l|}{ revolute } \\
\hline Active & \multicolumn{2}{|l|}{ - always $\subset$ kinematic mode } \\
\hline Location Dependency & \multicolumn{2}{|c|}{ Delta location from coordinate } \\
\hline Coordinate Reference & \multicolumn{2}{|c|}{ al_duploa.ground.cfl_upright_spindle_inr } \\
\hline Location & \multicolumn{2}{|l|}{$0,0,0$} \\
\hline Location in & \multicolumn{2}{|l|}{ - local $\subset$ global } \\
\hline Orientation Dependency & \multicolumn{2}{|c|}{ Parallel to axis } \\
\hline Construction Frame & \multicolumn{2}{|c|}{ I_duploa.ground.cfl_upright_spindle_inf } \\
\hline Axis on Entity & \multicolumn{2}{|l|}{$\sigma 2 \circ x$} \\
\hline Axis on Frame & \multicolumn{2}{|l|}{ (ब $+z<-z<+x<-x$} \\
\hline 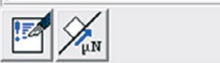 & Apply & Cancel \\
\hline
\end{tabular}

Figura 5.120 - Preenchimento da caixa de diálogo para criação da Junta 4. 


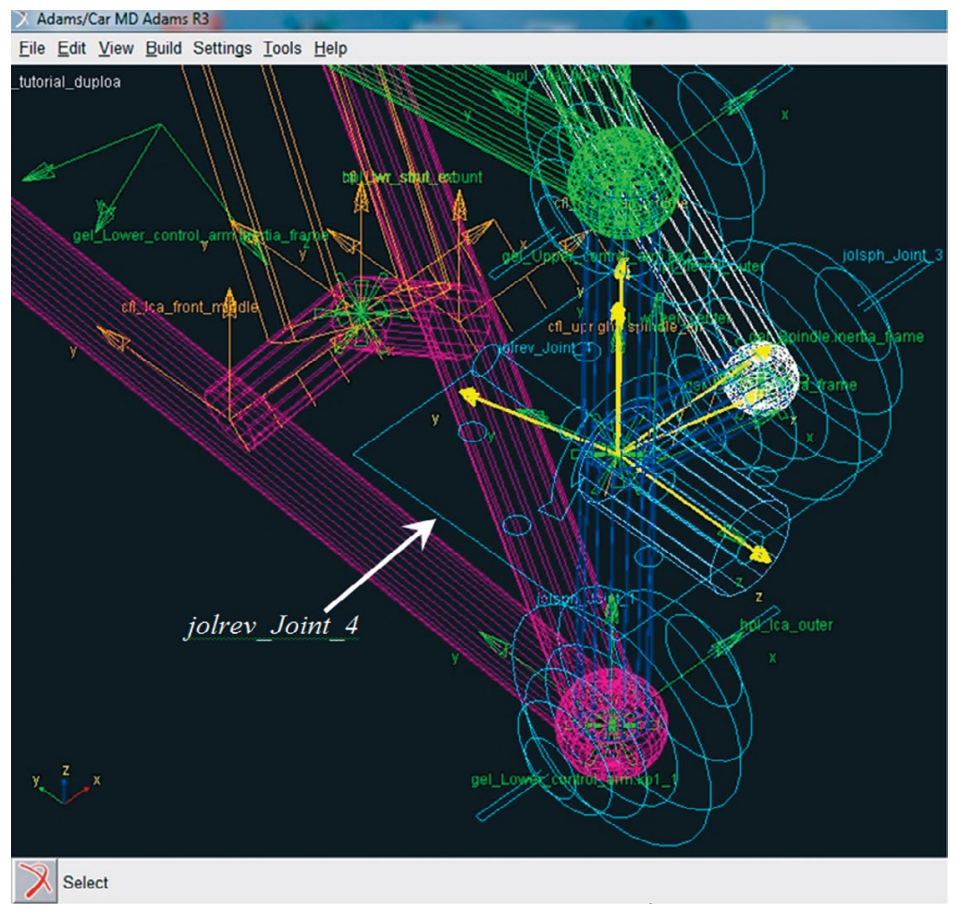

Figura 5.121 - Tela ilustrativa da criação da Junta 4 construída.

Etapa 19 - Junta de ligação entre os braços da suspensão e o chassi

Nesta etapa, será criado o par de juntas responsáveis pela restrição dos graus de liberdade entre o chassi e os braços de suspensão. Para inserção das juntas da suspensão, siga:

- $\quad$ Acesse o menu Build $\rightarrow$ Attachments $\rightarrow$ Joints $\rightarrow$ New (Figura 5.122). 


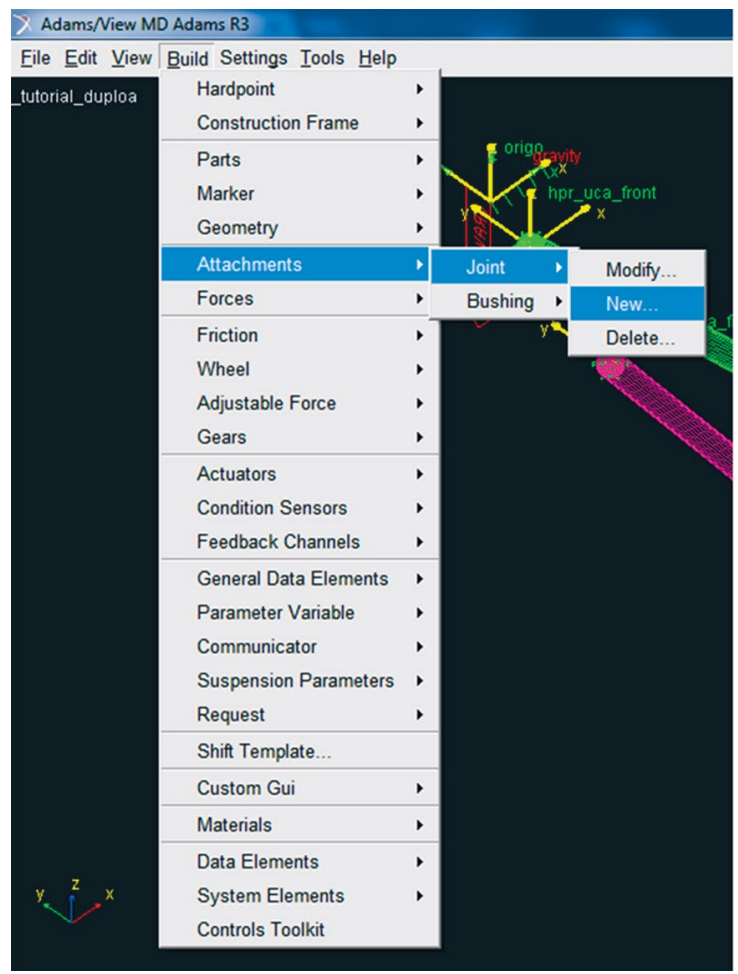

Figura 5.122 - Janela correspondente ao menu para criação de juntas.

- Na janela Create Joint Attachment, preencha os campos, conforme mostrado na Figura 5.123. Note que esta junta é do tipo revoluta (Revolute) e é construída entre o General Part "gel_Lower_control_arm" e o Mount "mtl_subframe_to_body".

- Após preenchimento sugerido, clique em $O K$.

A Figura 5.124 destaca a junta criada. 


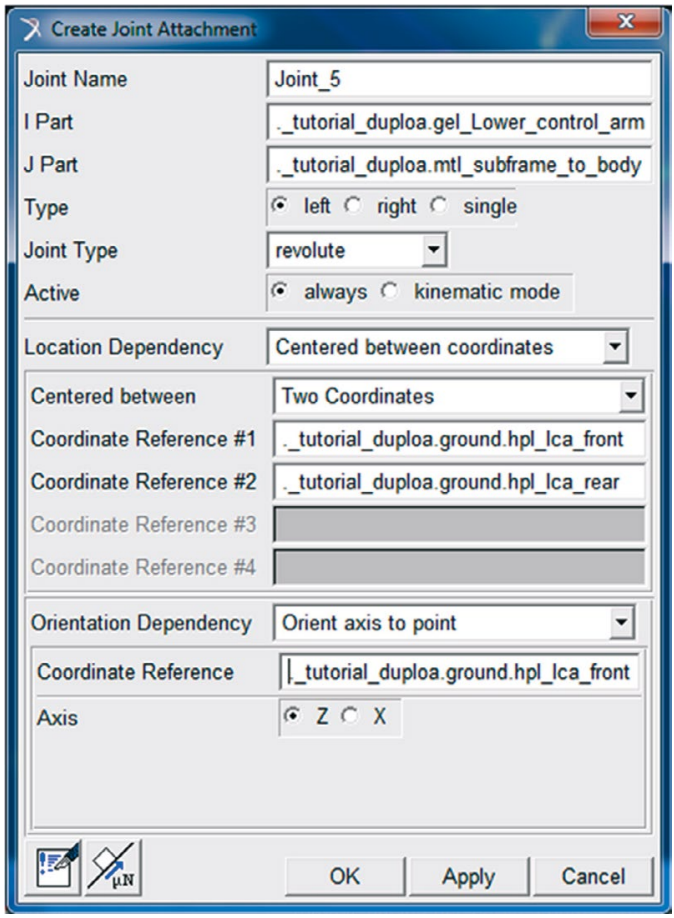

Figura 5.123 - Preenchimento de caixa de diálogo para criação da Junta 5 .

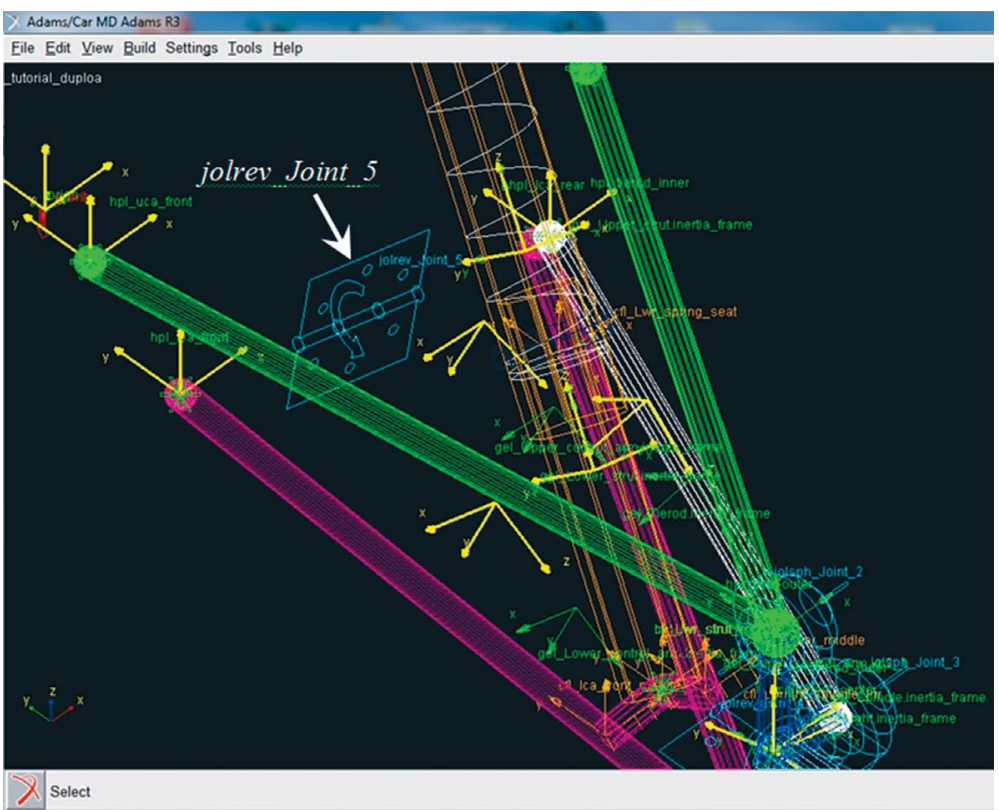

Figura 5.124 - Tela ilustrativa da Junta 5 construída. 
O procedimento descrito aqui deve, então, ser repetido para a bandeja superior da suspensão. Sendo assim, prossiga:

- $\quad \mathrm{Na}$ janela Create Joint Attachment, preencha os campos, conforme mostrado na Figura 5.125. Note que essa junta é do tipo revoluta e é construída entre o General Part "gel_Upper_control_arm" e o Mount "mtl_uca_to_body".

- $\quad$ Clique em $O K$.

- Salve seu projeto.

A Figura 5.126 destaca a junta criada, segundo a sugestão de preenchimento apresentada na Figura 5.125.

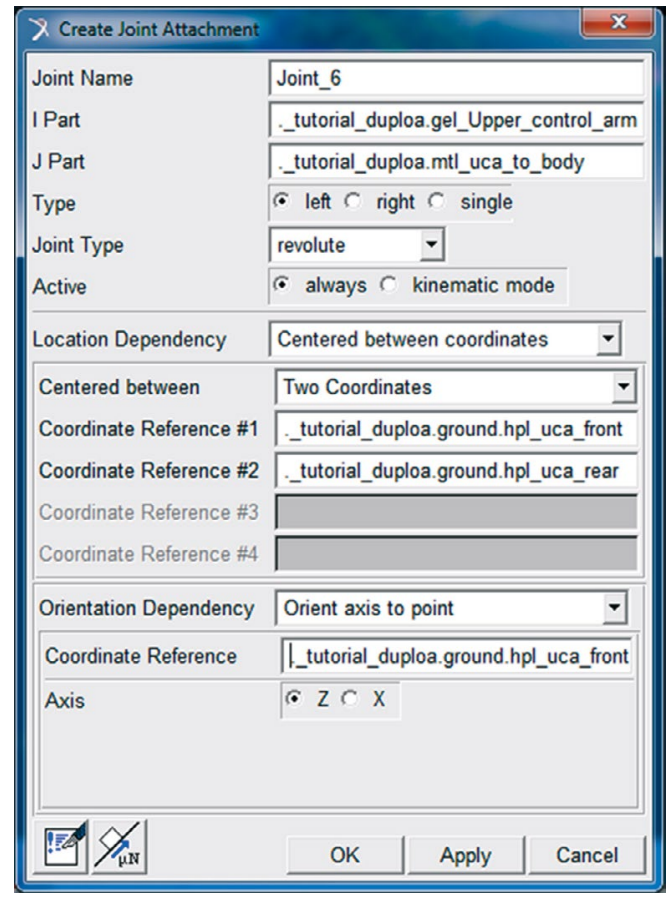

Figura 5.125 - Preenchimento de caixa de diálogo para criação da Junta 6 . 


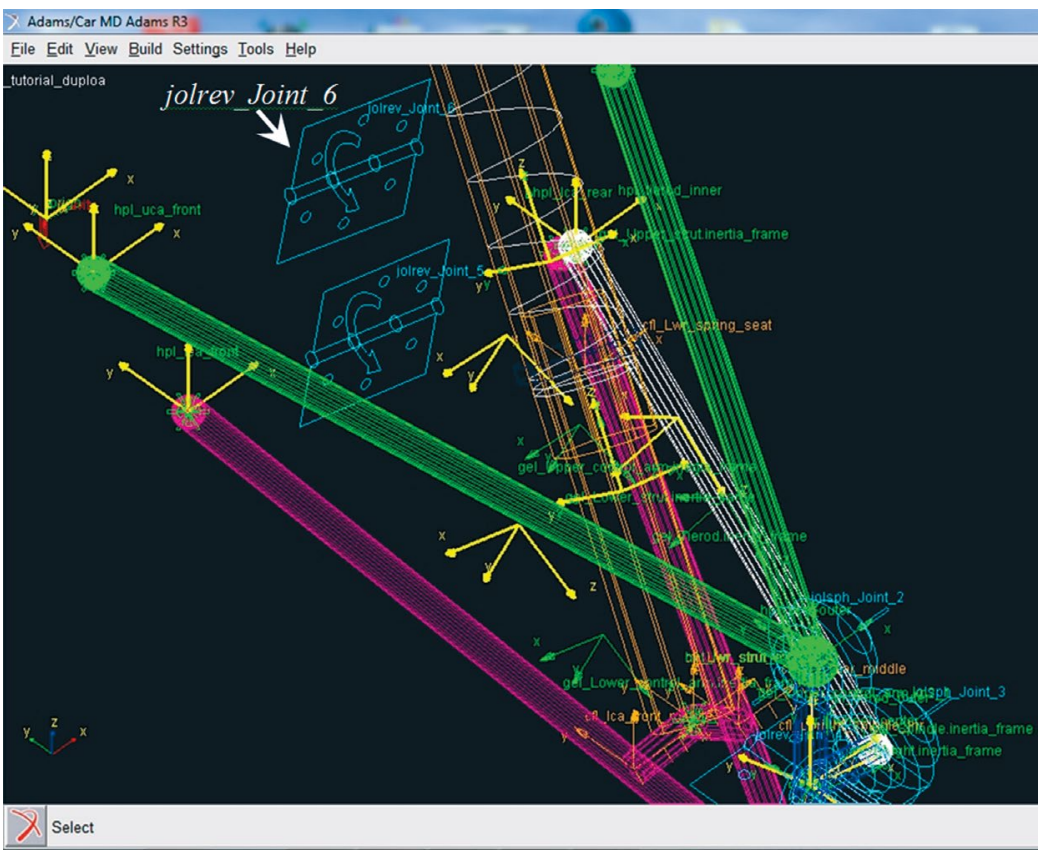

Figura 5.126 - Tela ilustrativa da Junta 6 criada.

\section{Etapa 20 - Criação da junta Hooke}

Nesta etapa, serão construídas duas juntas Hooke, as quais têm como coordenadas de referência (Coordinate Reference) os Hardpoint "hpl_lwr_strut_ mount" para a Junta 7 e “top_mount” para a junta 8 . Ambas possuem a função de, respectivamente, restringir graus de liberdade entre o braço e o amortecedor e entre o amortecedor e o chassi. Para cumprir esta Etapa:

- $\quad$ Acesse o menu Build $\rightarrow$ Attachments $\rightarrow$ Joint $\rightarrow$ New (Figura 5.127). 


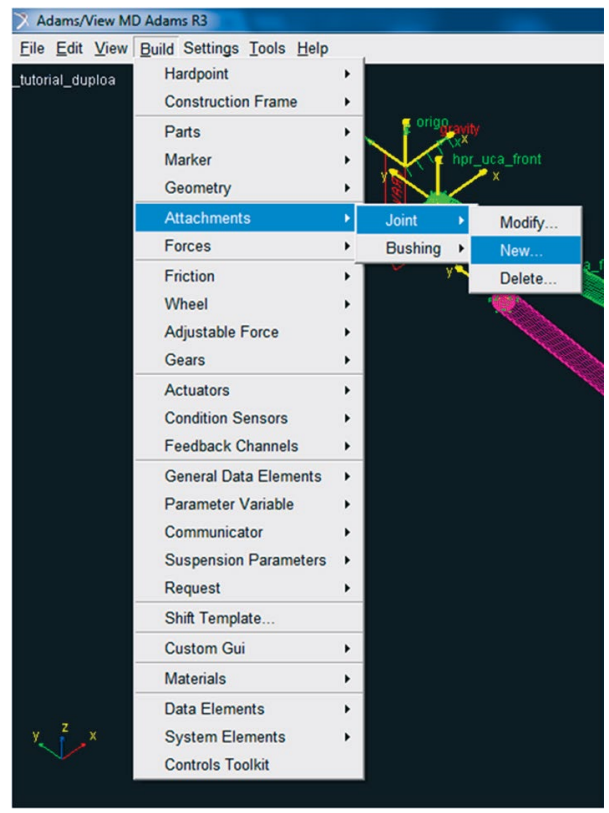

Figura 5.127 - Janela correspondente ao menu para criação da Junta 7 .

- Preencha os campos da janela Create Joint Attachment, de acordo com o mostrado na Figura 5.128.

Note que essa junta está sendo criada entre o General Part "gel_Lower_ strut” e o General Part “Lower_control_arm”.

- Clique em $O K$, ao finalizar.

A Figura 5.129 ilustra a junta Hooke construída, a partir do preenchimento da caixa de diálogo mostrada na Figura 5.128. 


\begin{tabular}{|c|c|}
\hline Create Joint Attachme & $x$ \\
\hline Joint Name & Joint_7 \\
\hline I Part & _tutorial_duploa.gel_Lower_strut \\
\hline J Part & _tutorial_duploa_gel_Lower_control_arm \\
\hline Type & $C$ left $C$ right $C$ single \\
\hline Joint Type & hooke \\
\hline Active & - always $\subset$ kinematic mode \\
\hline Location Dependency & Delta location from coordinate \\
\hline Coordinate Reference & prial_duploa.ground.hpl_lwr_strut_mount \\
\hline Location & $0,0,0$ \\
\hline Location in & (- local $\subset$ global \\
\hline I-Part Axis & -_tutorial_duploa.ground.hpl_top_mount \\
\hline J-Part Axis & tutorial_duploa.ground.cfl_Lwr_strut_ext \\
\hline FEd $/ \%_{\mu \mathrm{N}}$ & Cancel \\
\hline
\end{tabular}

Figura 5.128 - Preenchimento da caixa de diálogo para criação da junta 7 , do tipo Hooke.

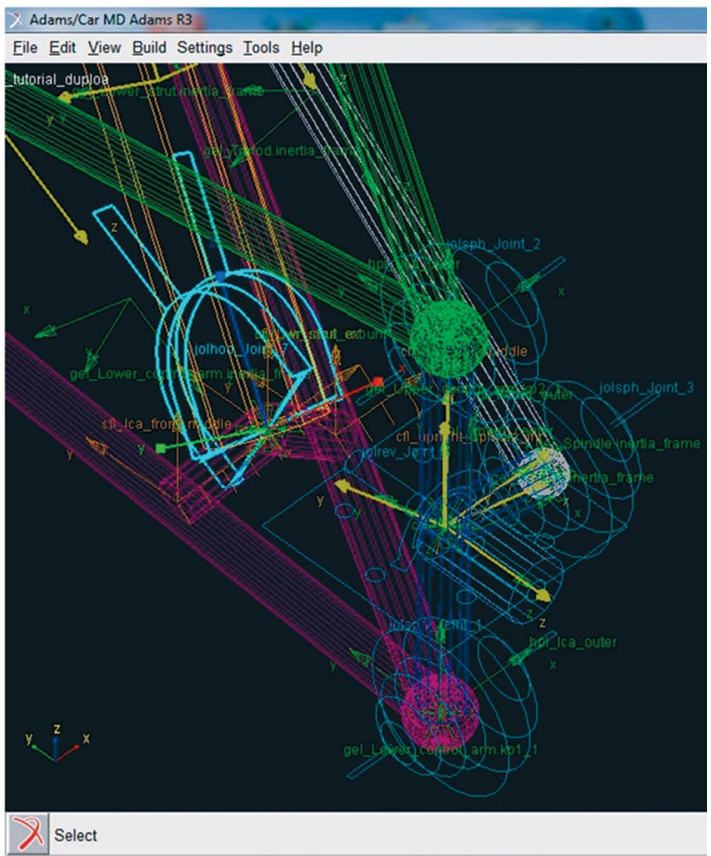

Figura 5.129 - Ilustração em destaque da junta Hooke construída. 
Para criação da junta que restringirá graus de liberdade entre o amortecedor e o chassi é necessário que o preenchimento dos campos da janela Create Joint Attachment esteja de acordo com a Figura 5.130. Está será entre o General Part "gel_Upper_strut" e o Mount "mtr_strut_to_body".

- $\quad$ Clique em OK.

- Salve seu projeto

A Figura 5.131 ilustra a junta Hooke construída.

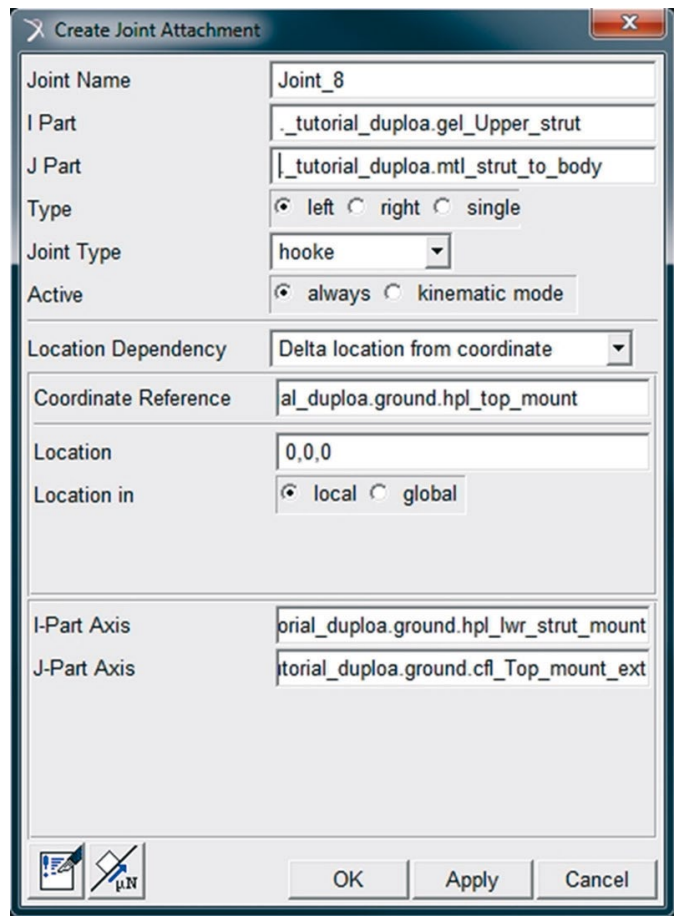

Figura 5.130 - Preenchimento da caixa de diálogo para criação da junta 8 tipo Hooke. 


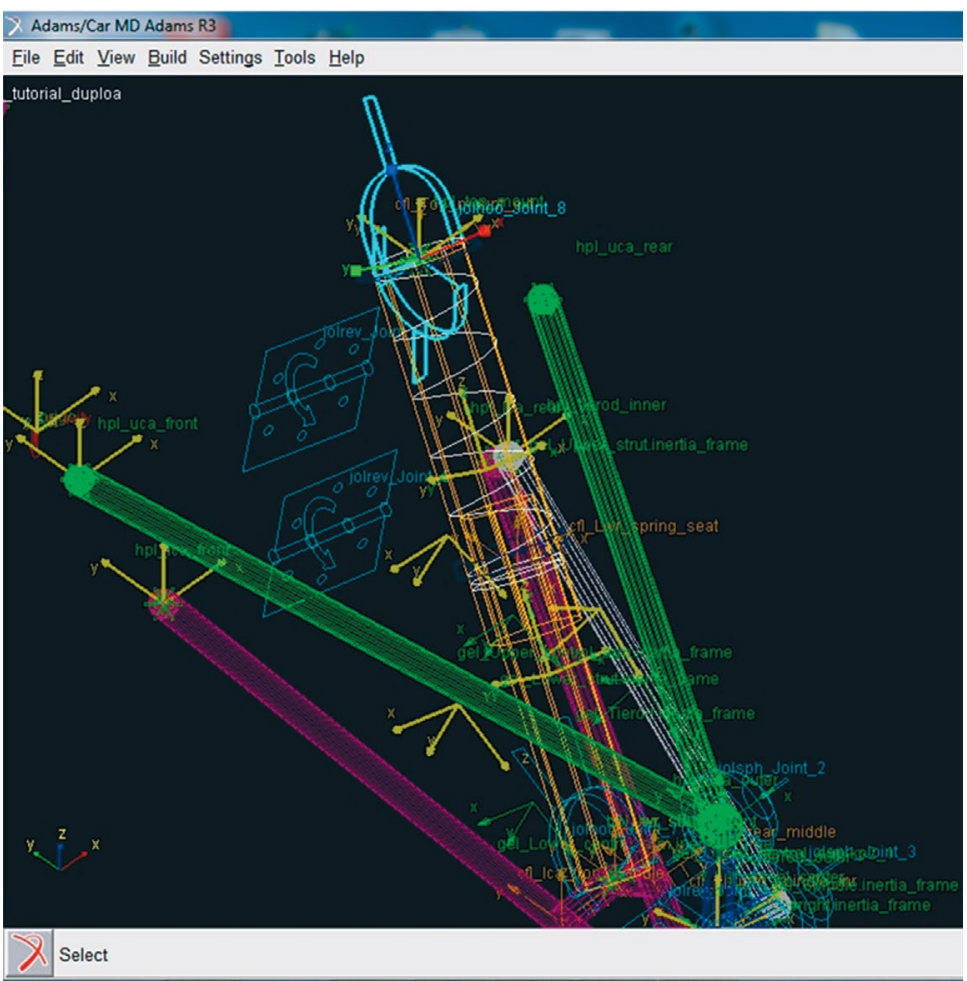

Figura 5.131 - Ilustração em destaque da junta Hooke construída.

Etapa 21 - Criação da junta no eixo de direção (Convel Joint)

Nesta etapa, será criada uma junta do tipo Convel, no eixo de direção (Tie rod). Essa junta restringirá os movimentos desse eixo no subsistema Sistema de Direção.

Para criar a referida junta no eixo de direção, proceda assim:

- Acesse o menu Build $\rightarrow$ Attachments $\rightarrow$ Joint $\rightarrow$ New (Figura 5.132). 


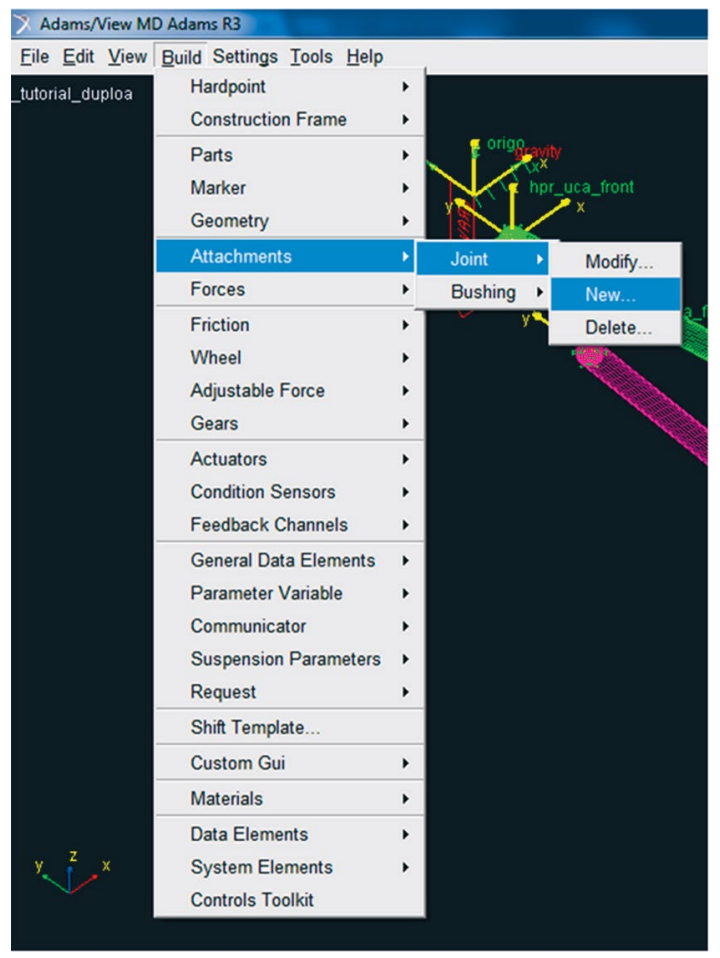

Figura 5.132 - Janela correspondente ao menu para criação da junta Convel.

- $\quad$ Crie a junta do tipo Convel, conforme ilustrado na Figura 5.133.

- Note que essa junta é definida pelo General Part "gel_tierod" e o Mount “mtl_tierod_to_steering”. Atente-se para o Hardpoint destacado na Figura 5.133, perceba que $J$ - Part Axis é um Hardpoint situado à direita.

- Após preenchimento, clique em $O K$.

- $\quad$ Salve seu projeto. 


\begin{tabular}{|c|c|}
\hline$X$ Create Joint Attachmer & $x$ \\
\hline Joint Name & Joint_10 \\
\hline I Part & -_tutorial_duploa.gel_Tierod \\
\hline J Part & -_tutorial_duploa.mtl_tierod_to_steering \\
\hline Type & (- left $C$ right $C$ single \\
\hline Joint Type & convel \\
\hline Active & C. always $\subset$ kinematic mode \\
\hline Location Dependency & Delta location from coordinate \\
\hline Coordinate Reference & _tutorial_duploa.ground.hpl_tierod_inner \\
\hline Location & $0,0,0$ \\
\hline Location in & - local $\odot$ global \\
\hline I-Part Axis & _tutorial_duploa.ground.hpl_tierod_outer \\
\hline J-Part Axis & _tutorial_duploa.groun_hpr_tierod_inner \\
\hline EEd $/ 4$ & Cancel \\
\hline
\end{tabular}

Figura 5.133 - Preenchimento da caixa de diálogo para criação da junta Convel.

\section{A Figura 5.134 ilustra a junta do eixo de direção construída.}

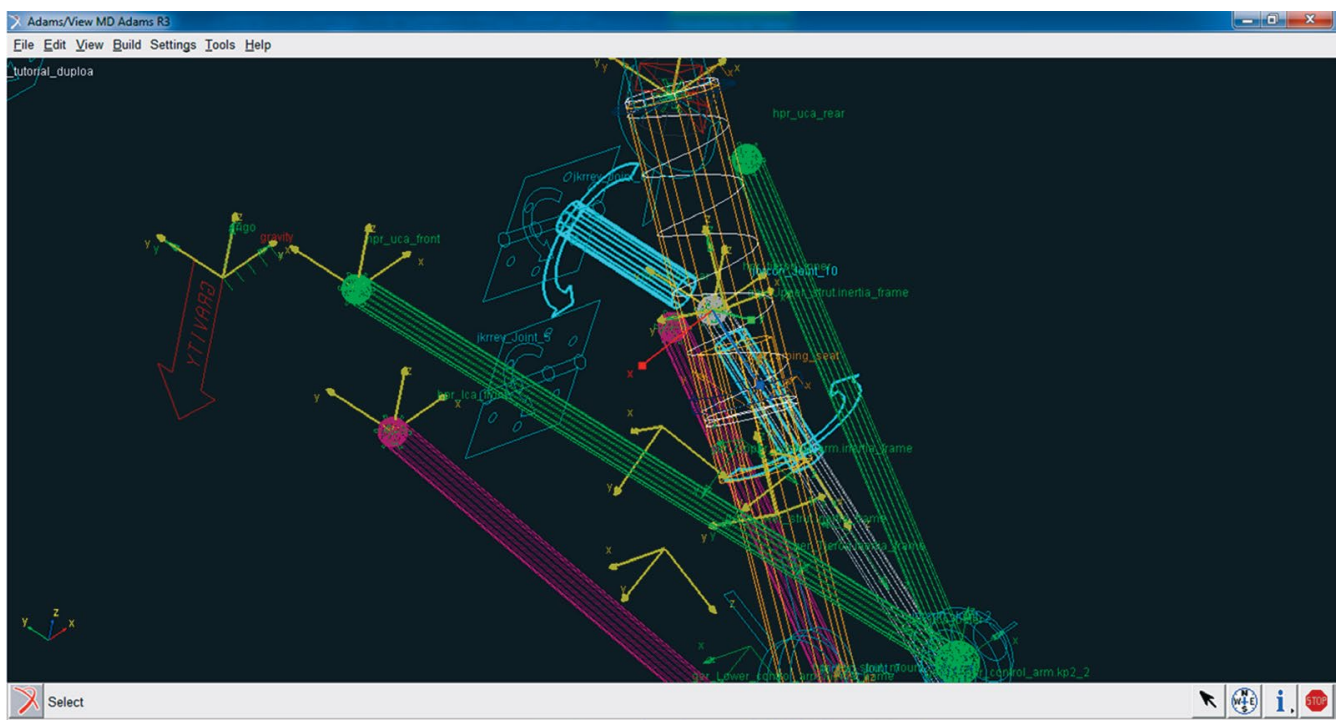

Figura 5.134 - Ilustração em destaque da junta Convel do eixo de direção construída. 


\section{Etapa 22 - Construção das junções por buchas (Bushing Attachments)}

Nesta etapa, serão criadas as buchas da suspensão em um total de quatro buchas entre o chassi e os braços da suspensão, ou seja, uma em cada extremidade do braço de suspensão e duas buchas nas extremidades do amortecedor, que se fixam ao chassi.

- Para criar a primeira bucha, a qual estará localizada na mesma coordenada que o Hardpoint " $h p l \_l c a \_f r o n t$ ", acesse o menu Build $\rightarrow$ Attachments $\rightarrow$ Bushing $\rightarrow$ New (Figura 5.135).

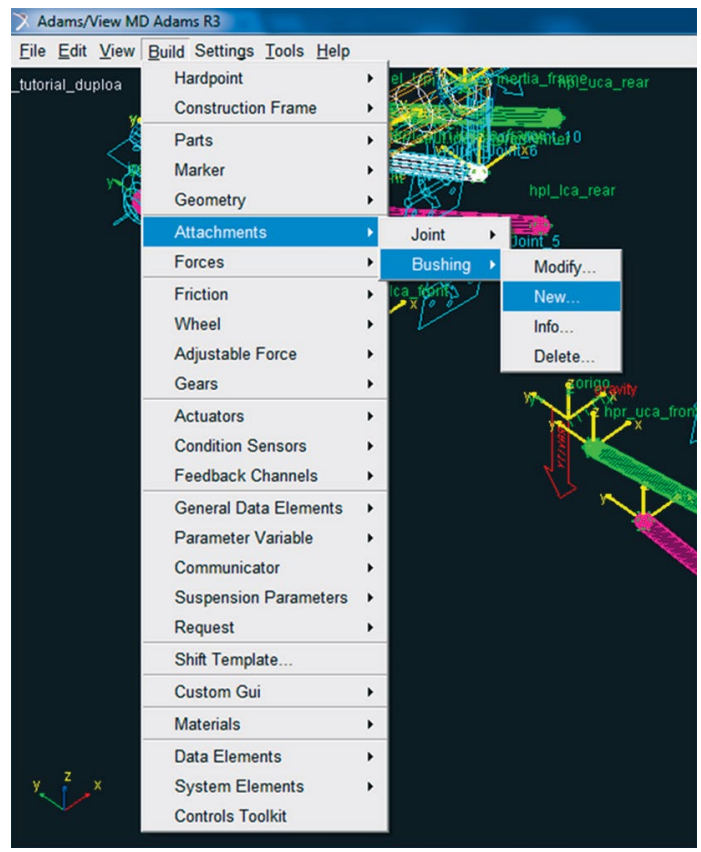

Figura 5.135 - Janela correspondente ao menu de criação da bucha "bkl_Bushing1".

Os campos da janela Create Bushing Attachment deverão ser preenchidos, conforme a Figura 5.136.

Note que essa bucha é criada entre o General Part "gel_Lower_control_arm" e o Mount "mtl_subframe_to_body". Um arquivo com as propriedades da bucha deve ser selecionado (no campo Property File), podendo este ser da biblioteca do software ou editado pelo usuário.

- $\quad$ Clique em $O K$. 


\begin{tabular}{|c|c|}
\hline \multicolumn{2}{|c|}{ Create Bushing Attachment } \\
\hline Bushing Name & Bushing1 \\
\hline I Part & -_tutorial_duploa.gel_Lower_control_arm \\
\hline J Part & -tutorial_duploa.mtl_subframe_to_body \\
\hline Type & (- left $C$ right $C$ single \\
\hline Inactive & C never $\cdot$ kinematic mode \\
\hline Preload & $0,0,0$ \\
\hline Tpreload & $0,0,0$ \\
\hline Offset & $0,0,0$ \\
\hline Roffset & $0,0,0$ \\
\hline Geometry Length & 40 \\
\hline Geometry Radius & 25 \\
\hline Property File & //acar_shared/bushings.tbl/mdi_0001.bus \\
\hline Location Dependency & Delta location from coordinate \\
\hline Coordinate Reference & -_tutorial_duploa.ground.hpl_Ica_front \\
\hline Location & $0,0,0$ \\
\hline Location in & - local $C$ global \\
\hline Orientation Dependency & Orient axis along line \\
\hline Coordinate Reference \#1 & -_tutorial_duploa.ground.hpl_Ica_front \\
\hline Coordinate Reference \#2 & _.tutorial_duploa.ground.hpl_Ica_rear \\
\hline Axis & • $z \sim x$ \\
\hline EF & Cancel \\
\hline
\end{tabular}

Figura 5.136 - Preenchimento da caixa de diálogo para criação da bucha "bkl_Bushing1".

\section{A Figura 5.137 ilustra a bucha criada no passo anterior.}

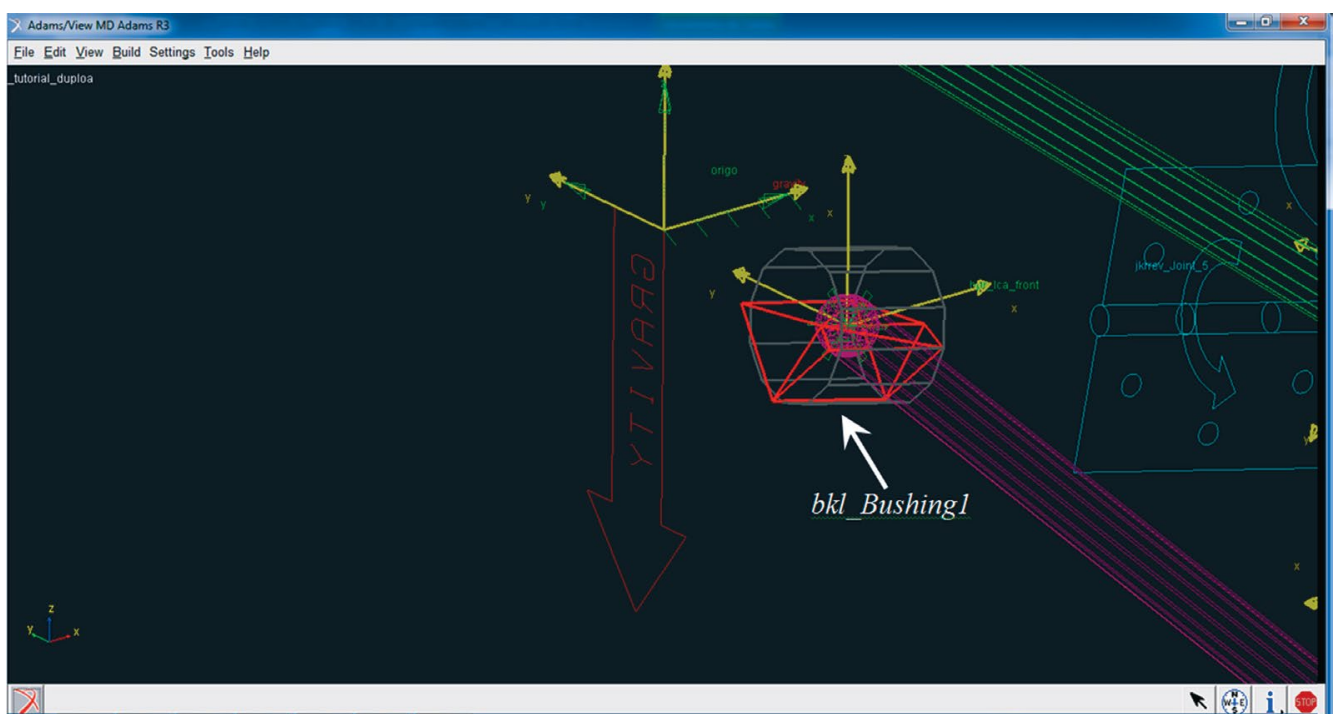

Figura 5.137 - Ilustração da bucha “bkl_Bushing1” inserida no modelo virtual. 
Para construção da segunda bucha, a qual estará localizada no Hardpoint hpl_lca_rear:

- Acesse o menu Build $\rightarrow$ Attachments $\rightarrow$ Bushing $\rightarrow$ New (Figura 5.138).

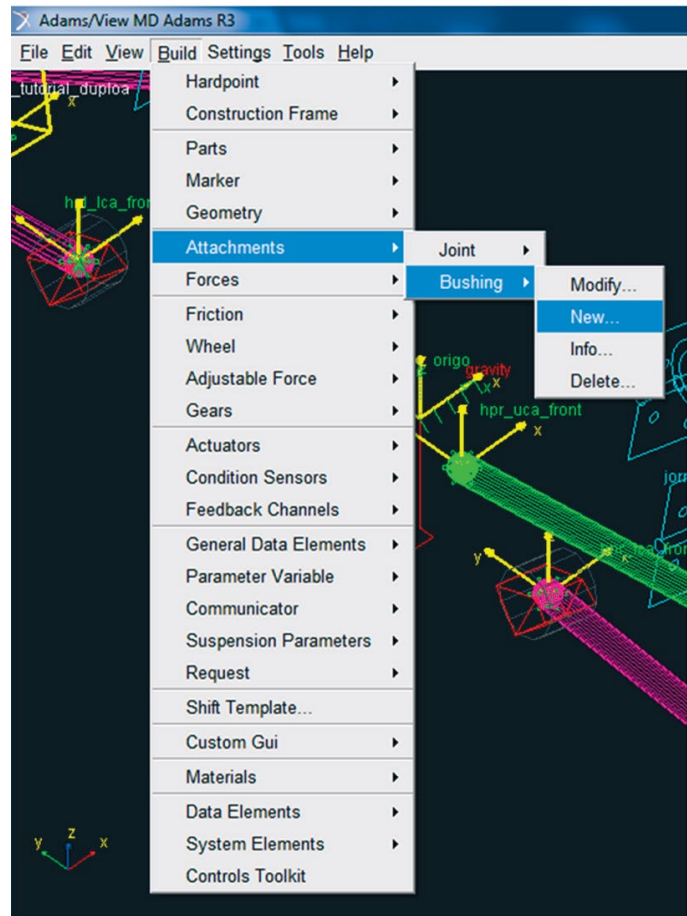

Figura 5.138 - Janela correspondente ao menu de criação da bucha "bkl_Bushing2".

- Preencha os campos da janela Create Bushing Attachment, como ilustrado na Figura 5.139.

- $\quad$ Clique em $O K$.

Note que essa bucha também é construída entre o General Part "gel_Lower_control_arm" e o Mount "mtl_subframe_to_body". 


\begin{tabular}{|c|c|}
\hline \multicolumn{2}{|c|}{ Create Bushing Attachment } \\
\hline Bushing Name & Bushing2 \\
\hline I Part & -_tutorial_duploa.gel_Lower_control_arm \\
\hline$J$ Part & -_tutorial_duploa.mtl_subframe_to_body \\
\hline Type & - left $\subset$ right $\subset$ single \\
\hline Inactive & $S$ never 5 kinematic mode \\
\hline Preload & 0.0 .0 \\
\hline Tpreload & $0,0,0$ \\
\hline Offset & 0.0 .0 \\
\hline Roffset & $0,0,0$ \\
\hline Geometry Length & 40 \\
\hline Geometry Radius & 25 \\
\hline Property File & //acar_shared/bushings.tbl/mdi_0001.bus \\
\hline Location Dependency & Delta location from coordinate \\
\hline Coordinate Reference & _tutorial_duploa_ground.hpl_Ica_rear \\
\hline Location & $0,0,0$ \\
\hline Location in & - local C global \\
\hline Orientation Dependency & Orient axis along line \\
\hline Coordinate Reference \#1 & __tutorial_duploa.ground.hpl_Ica_front \\
\hline Coordinate Reference \#2 & -_tutorial_duploa.ground.hpl_lca_rear \\
\hline Axis & $\Leftrightarrow 2 \circ x$ \\
\hline FEd Do & Cancel \\
\hline
\end{tabular}

Figura 5.139 - Preenchimento da caixa de diálogo relativa à criação da bucha "bkl_Bushing2".

A Figura 5.140 ilustra a segunda bucha “bkl_Bushing2” construída.

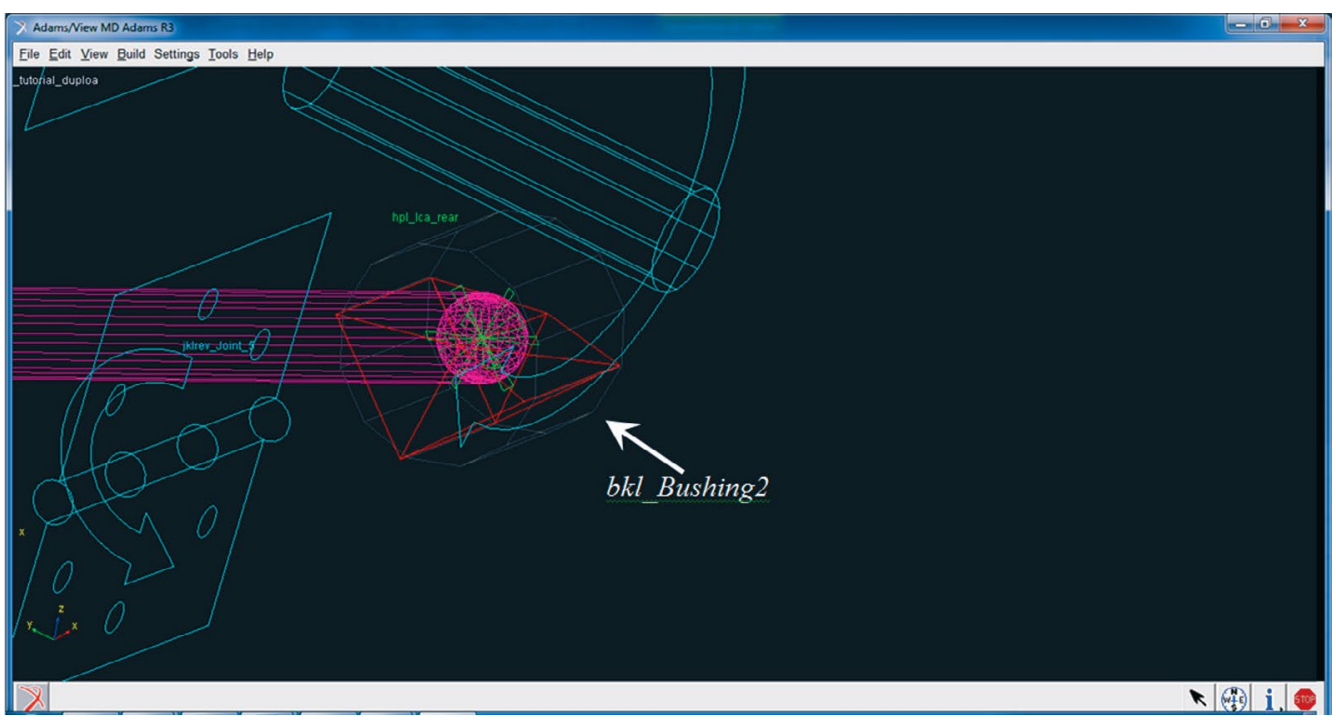

Figura 5.140 - Ilustração da segunda bucha “bkl_Bushing2” inserida no modelo. 
- Para construção da terceira bucha, a qual estará localizada no hardpoint $h p l \_u c a \_f r o n t$ repita os procedimentos anteriores e preencha os campos da janela Create Bushing Attachment como ilustrado na Figura 5.141.

- Terminado preenchimento, clique em $O K$.

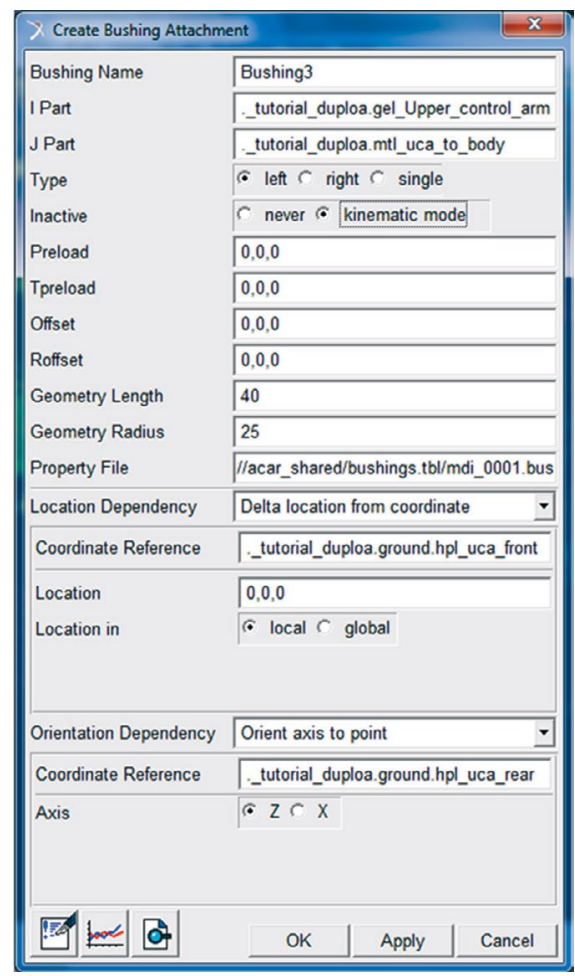

Figura 5.141 - Preenchimento da caixa de diálogo relativa à criação da bucha " $b k l_{-}$ Bushing3”.

A Figura 5.142 ilustra a bucha “bkl_Bushing3” construída. 


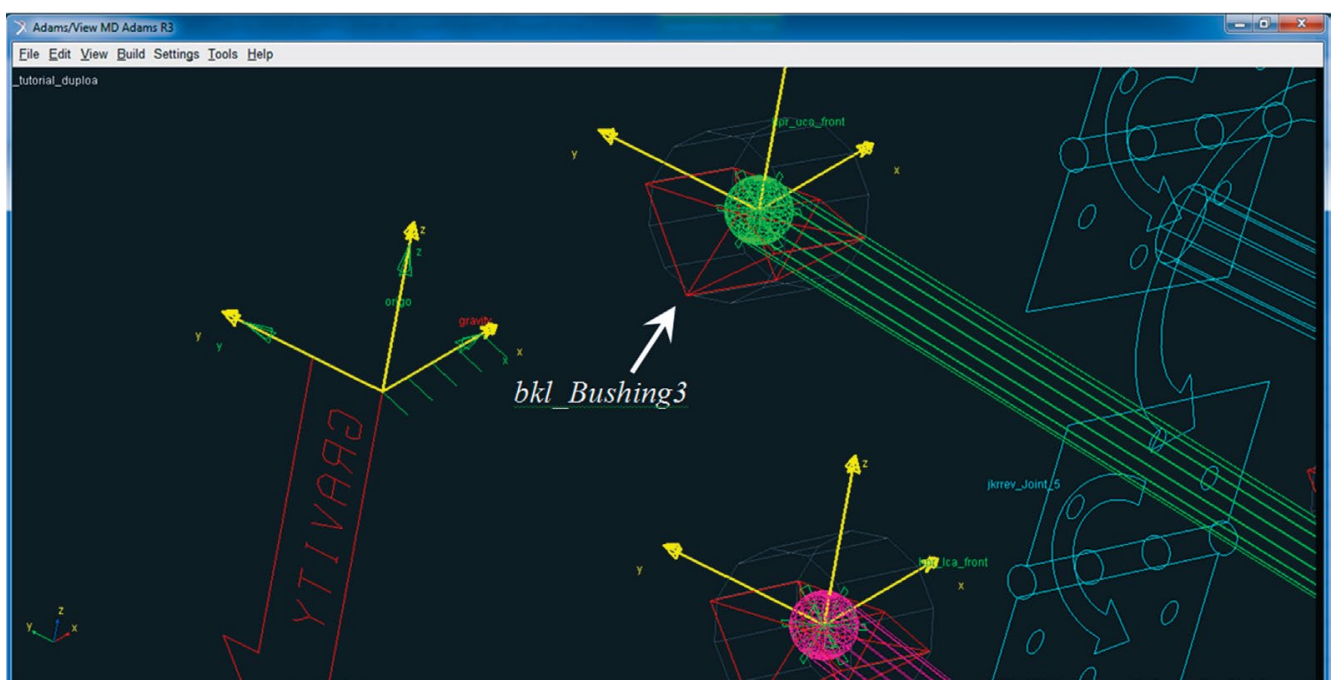

Figura 5.142 - Ilustração da terceira bucha “bkl_Bushing3” inserida no modelo.

A construção da quarta bucha seguirá o mesmo procedimento das três anteriores.

- Repita os procedimentos e preencha os campos da janela Create Bushing Attachment, como ilustrado na Figura 5.143.

- $\quad$ Em seguida, clique em $O K$. 


\begin{tabular}{|c|c|}
\hline \multicolumn{2}{|c|}{ Create Bushing Attachment } \\
\hline Bushing Name & Bushing4 \\
\hline I Part & -_tutorial_duploa.gel_Upper_control_arm \\
\hline J Part & -_tutorial_duploa.mtl_uca_to_body \\
\hline Type & (- left $\subset$ right $C$ single \\
\hline Inactive & $S$ never 5 kinematic mode \\
\hline Preload & $0,0,0$ \\
\hline Tpreload & $0,0,0$ \\
\hline Offset & $0,0,0$ \\
\hline Roffset & $0,0,0$ \\
\hline Geometry Length & 40 \\
\hline Geometry Radius & 25 \\
\hline Property File & //acar_shared/bushings.tbl/mdi_0001.bus \\
\hline Location Dependency & Delta location from coordinate \\
\hline Coordinate Reference & _tutorial_duploa.ground.hpl_uca_rear \\
\hline Location & $0,0,0$ \\
\hline Location in & - local $\odot$ global \\
\hline Orientation Dependency & Orient axis to point \\
\hline Coordinate Reference & _tutorial_duploa.ground.hpl_uca_front \\
\hline Axis & C $\mathrm{ZCX}$ \\
\hline एहa boos & Cancel \\
\hline
\end{tabular}

Figura 5.143 - Preenchimento da caixa de diálogo relativa à criação da bucha " $b k l_{-}$ Bushing4".

A Figura 5.144 ilustra a quarta bucha construída " $b k l \_B u s h i n g 4$ ”. 


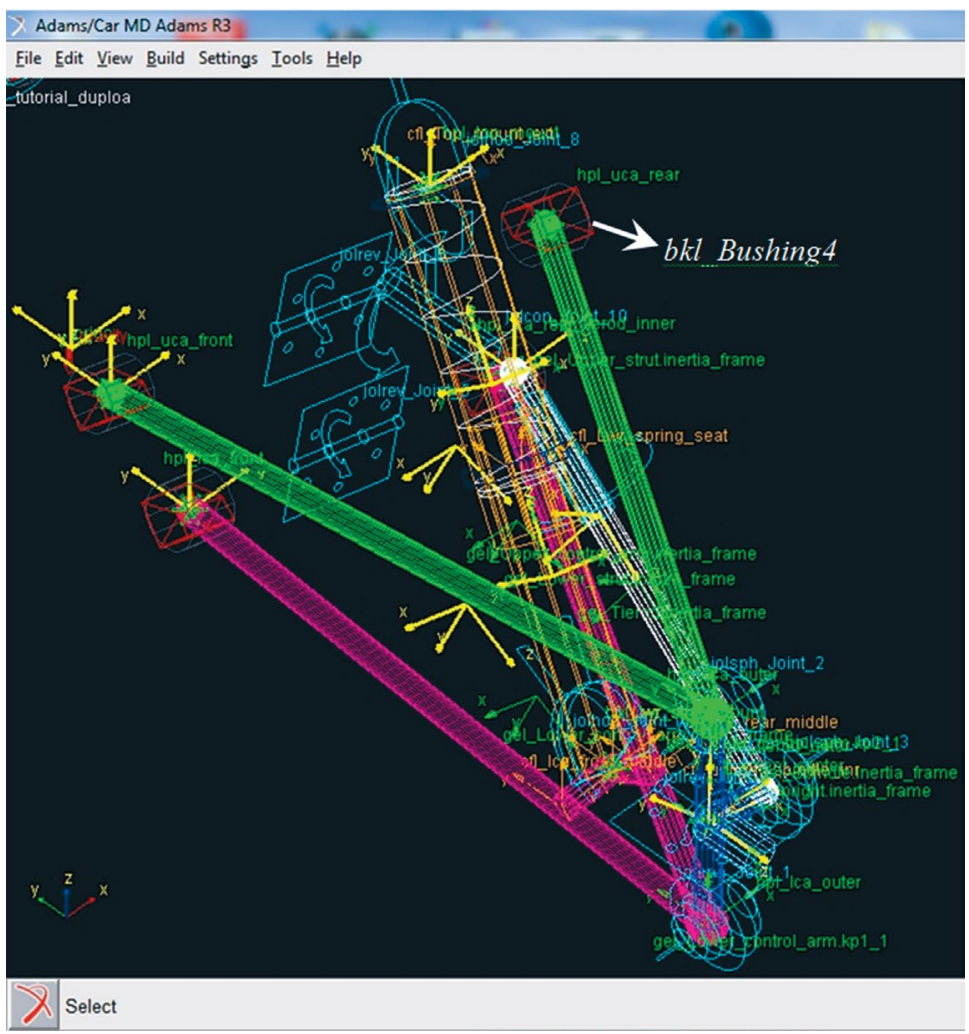

Figura 5.144 - Ilustração da quarta bucha “bkl_Bushing4" inserida no modelo.

Para a construção da quinta bucha:

- Preencha os campos da janela Create Bushing Attachment, como ilustrado na Figura 5.145.

- Finalizada a operação, clique em $O K$. 


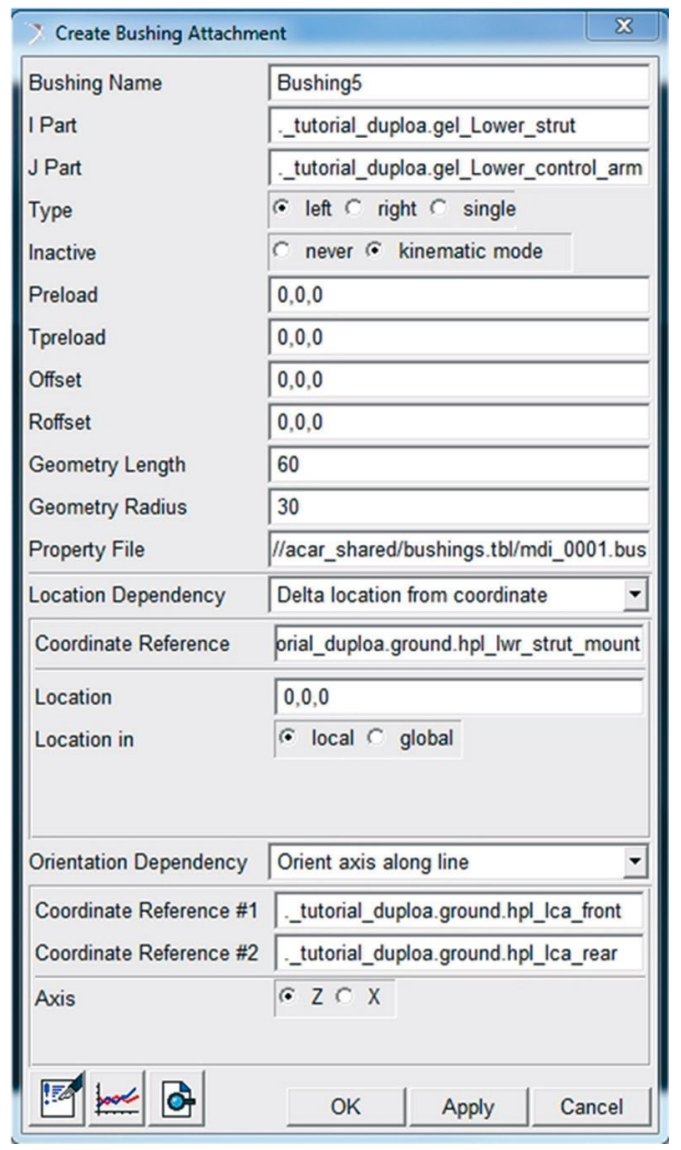

Figura 5.145 - Preenchimento da caixa de diálogo relativa à criação da bucha "bkl_Bushing5".

A Figura 5.146 ilustra a quinta bucha construída “bkl_Bushing5”. 


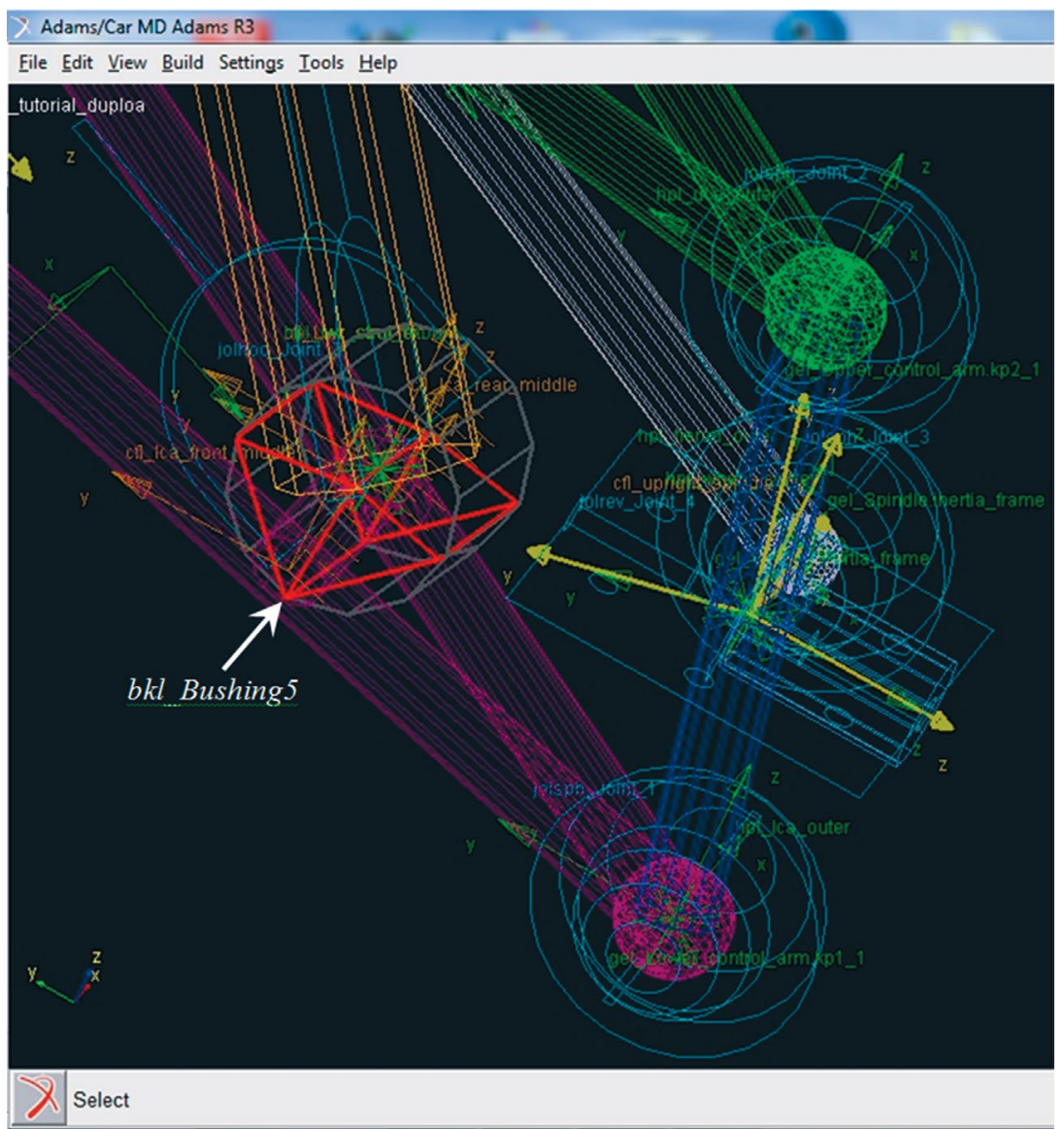

Figura 5.146 - Ilustração da quinta bucha “bkl_Bushing5” inserida no modelo.

- Para finalizar, a construção da sexta bucha se dá da mesma forma que a das cinco anteriores.

- Preencha os campos da janela Create Bushing Attachment, como ilustrado na Figura 5.147.

- $\quad$ Clique em $O K$.

- $\quad$ Salve seu projeto. 


\begin{tabular}{|c|c|}
\hline \multicolumn{2}{|c|}{ Create Bushing Attachment } \\
\hline Bushing Name & Bushing6 \\
\hline I Part & -_tutorial_duploa.gel_Upper_strut \\
\hline J Part & ._tutorial_duploa.mtl_strut_to_body \\
\hline Type & (- left $C$ right $\subset$ single \\
\hline Inactive & $S$ never 6 kinematic mode \\
\hline Preload & $0,0,0$ \\
\hline Tpreload & $0,0,0$ \\
\hline Offset & $0,0,0$ \\
\hline Roffset & $0,0,0$ \\
\hline Geometry Length & 20 \\
\hline Geometry Radius & 30 \\
\hline Property File & //acar_shared/bushings.tbl/mdi_0001.bus \\
\hline Location Dependency & Delta location from coordinate \\
\hline Coordinate Reference & -_tutorial_duploa.ground.hpl_top_mount \\
\hline Location & $0,0,0$ \\
\hline Location in & - local $\subset$ global \\
\hline Orientation Dependency & User entered values \\
\hline Orient using & - Euler Angles $C$ Direction Vectors \\
\hline Euler Angles & $0,0,0$ \\
\hline X Vector & $1.0,0.0,0.0$ \\
\hline Z Vector & $0.0,0.0,1.0$ \\
\hline 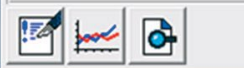 & Cancel \\
\hline
\end{tabular}

Figura 5.147 - Preenchimento da caixa de diálogo relativa à criação da bucha "bkl_Bushing6".

A Figura 5.148 ilustra a sexta bucha construída "bkl_Bushing6". 


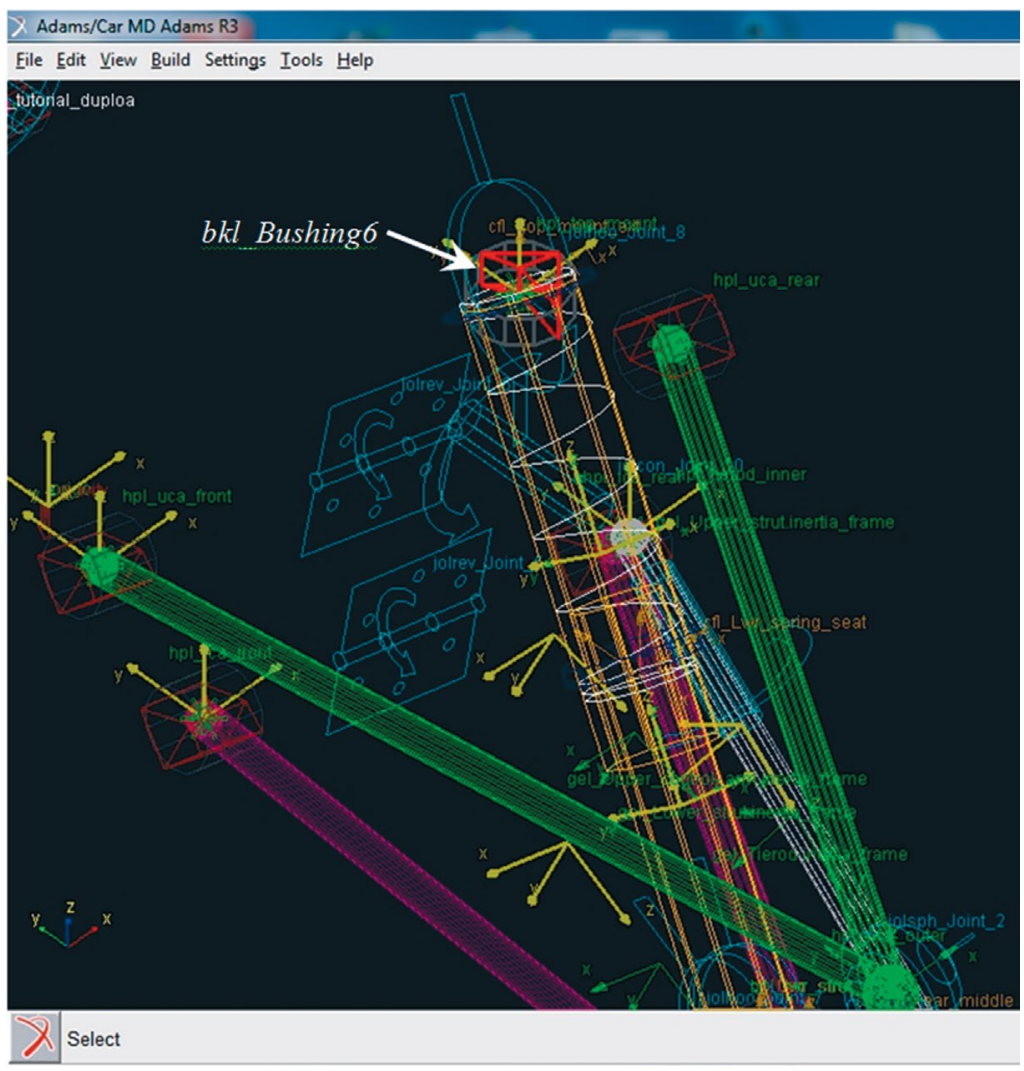

Figura 5.148 - Ilustração da sexta bucha "bkl_Bushing6" inserida no modelo.

\section{Etapa 23 - Criação de Communicators}

Nesta etapa, serão criados os Communicators do tipo Output, os quais estabelecerão a comunicação do subsistema suspensão com os diversos subsistemas que se acoplarão a este (Roda, Sistema de Direção, entre outros). Resalta-se, novamente, que os nomes designados aos Communicators devem ser iguais aos dos Communicators dos susbsistemas de interesse. Outro aspecto a considerar é que um Communicator output se relacionará com um Communicator input de outro subsistema.

Para criação do primeiro Communicator do tipo Output, proceda conforme especificado abaixo:

- $\quad$ Acesse o menu Build $\rightarrow$ Communicator $\rightarrow$ Output New (Figura 5.149). Este Communicator fará a comunicação entre o General Part "Uprigtht" (cubo de roda) e o subsistema roda. 


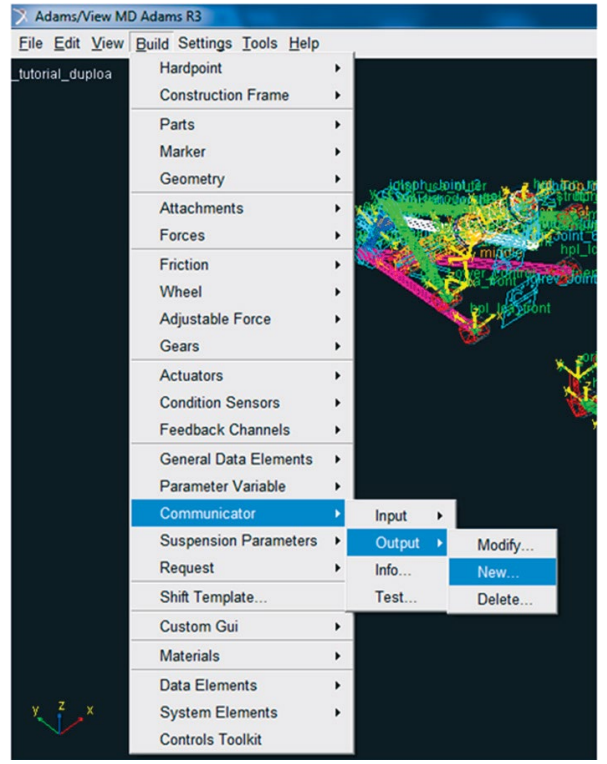

Figura 5.149 - Criando um Communicator do tipo Output.

A janela Create Output Communicator deverá ser preenchida conforme ilustrado na Figura 5.150.

- $\quad$ Clique em Apply.

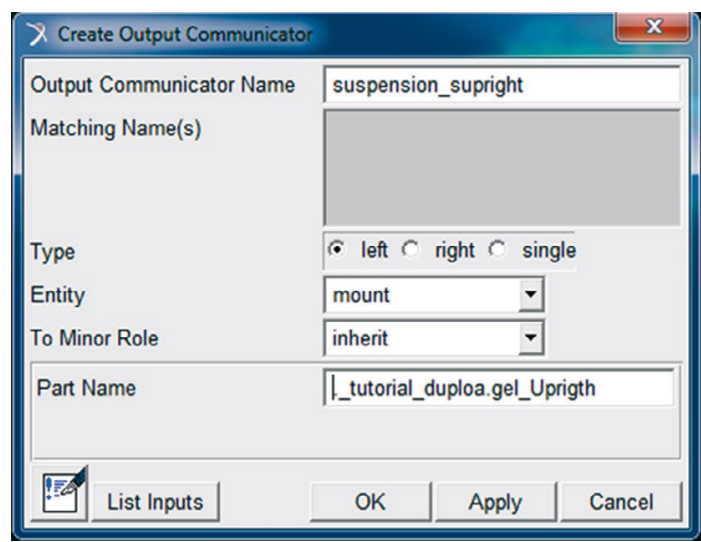

Figura 5.150 - Criação do Communicator "suspension_suprigth".

\section{Para criação do Communicator "suspension_mount":}

- Preencha os campos da janela Create Output Communicator, conforme ilustrado na Figura 5.151.

- $\quad$ Clique em Apply. 


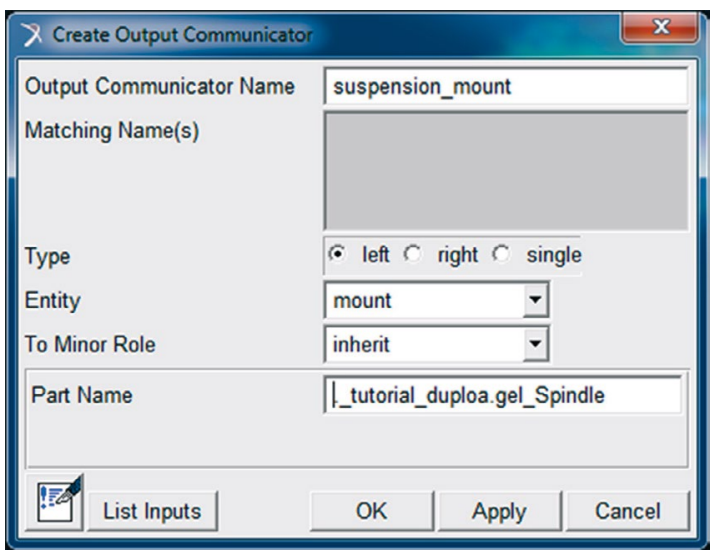

Figura 5.151 - Criação do Communicator "suspension_mount".

A Figura 5.152 ilustra a criação do Communicator "wheel_center".

- $\quad$ Clique em $O K$.

- $\quad$ Salve seu projeto.

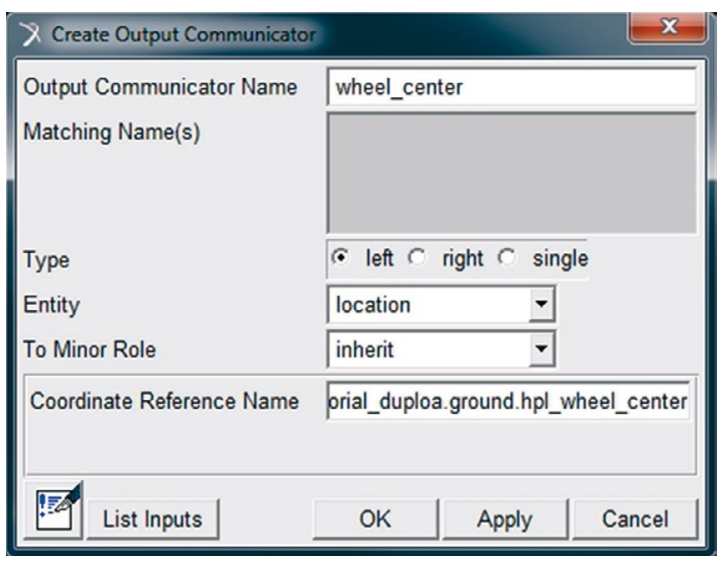

Figura 5.152 - Criação do Communicator "wheel_center".

\section{Etapa 24 - Realizando teste de compatibilidade entre Communicators}

Nesta etapa, serão obtidas informações sobre os Communicators criados, e ainda será feito um teste de compatibilidade entre estes e os Communicators do subsistema de interesse. Nesse caso, o subsistema de interesse é o MDI_Suspension_Testrig, o qual corresponde ao subsistema de teste da suspensão.

- Para se obter informações sobre os Communicators criados, acesse o menu Build $\rightarrow$ Communicator $\rightarrow$ Info (Figura 5.153). 


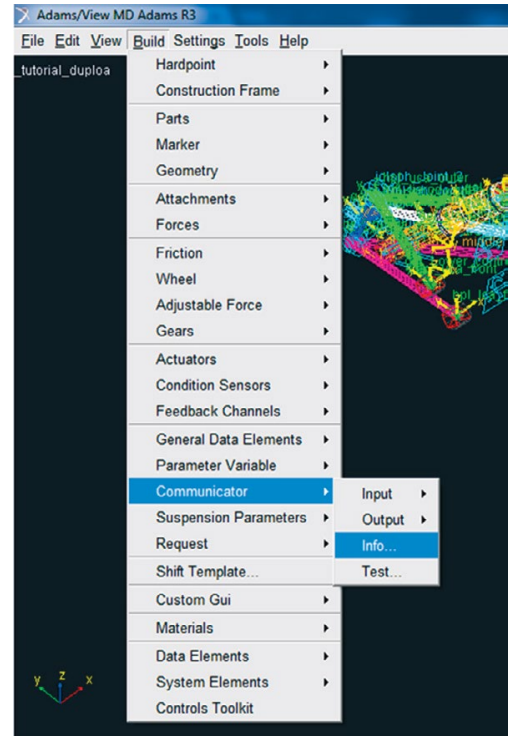

Figura 5.153 - Obtendo informações sobre os Communicators criados na Etapa 23.

A janela Communicators Info será aberta e se encontra ilustrada na Figura 5.154 .

No campo Model names aparece automaticamente o nome do Template que se está trabalhando, no caso,_tutorial_duploa.

- No campo Type, selecione o item All.

- No campo Entity selecione o item All.

- $\quad$ Clique em $O K$.

\begin{tabular}{|c|c|c|}
\hline \multicolumn{2}{|l|}{$X$ Communicators Info } & $x$ \\
\hline Model Names & \multicolumn{2}{|c|}{-_tutorial_duploa } \\
\hline C Inpu & Output $\odot$ All & \\
\hline \multicolumn{3}{|l|}{ Entity $\subset$ Many $\odot$ All } \\
\hline$\nabla$ array & $\nabla$ motion & \\
\hline F bushing & $F$ mount & \\
\hline V differential equation & V orientation & \\
\hline IV force & F parameter integer & \\
\hline V joint for motion & $\nabla$ parameter real & \\
\hline V joint & F part & \\
\hline IV location & V solver variable & \\
\hline V marker & F spline & \\
\hline \multicolumn{3}{|c|}{ V Clear Information Window } \\
\hline \multicolumn{3}{|l|}{$\ulcorner$ Save Info Results to File } \\
\hline \multicolumn{3}{|l|}{ File Name } \\
\hline & Apply & Cancel \\
\hline
\end{tabular}

Figura 5.154 - Selecionando os tipos de Communicators. 
A janela Information será aberta, e nela constam as informações requeridas, conforme a Figura 5.155.

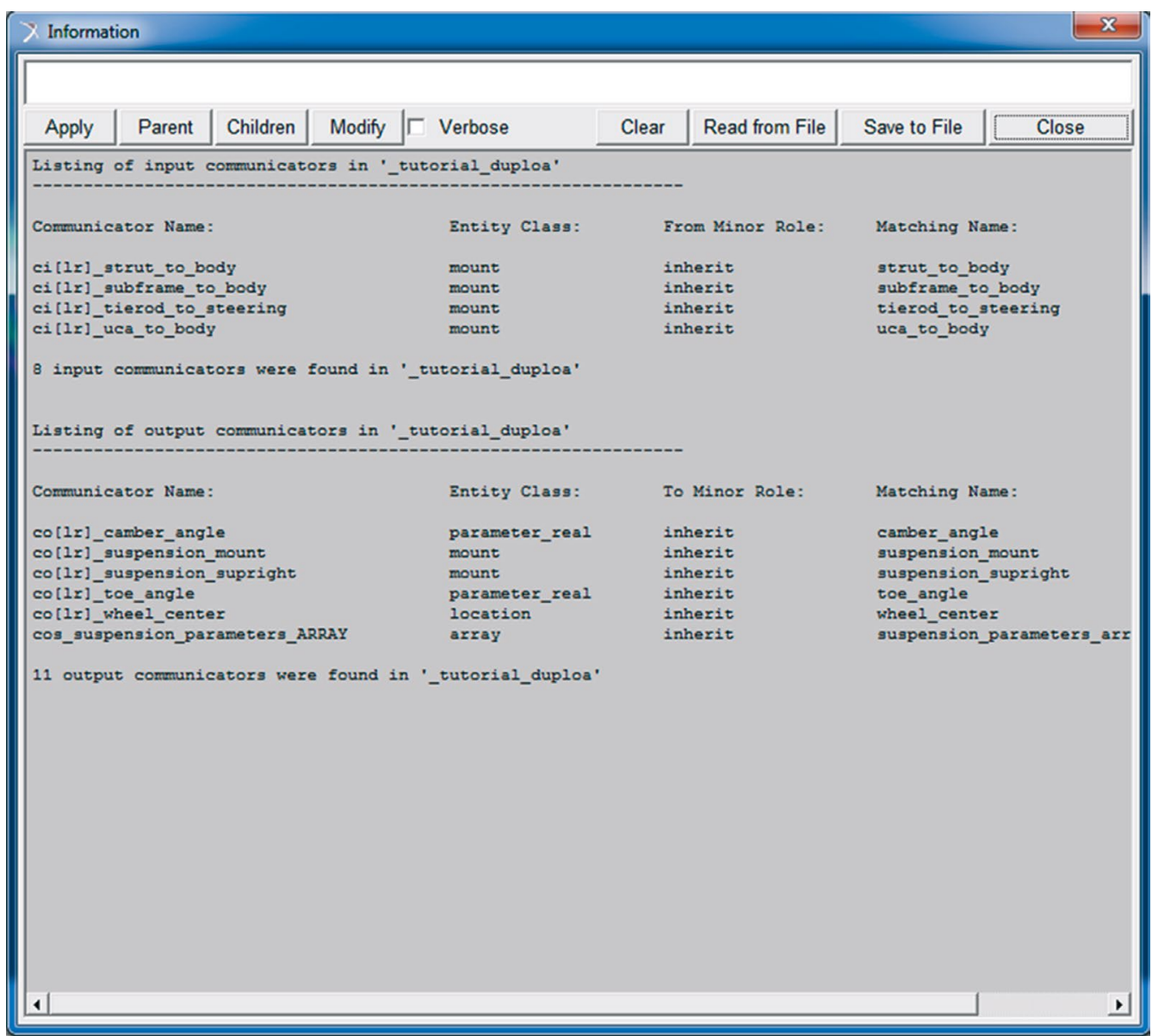

Figura 5.155 - Informações sobre os Communicators.

- Após análise das informações, clique em Close nesta mesma janela, opção que se encontra no canto superior direito da caixa de diálogo da Figura 5.155.

- Para teste de compatibilidade dos Communicators com o susbsistema MDI_Suspension_Testrig, acesse o menu Build $\rightarrow$ Communicator $\rightarrow$ Test (Figura 5.156). 


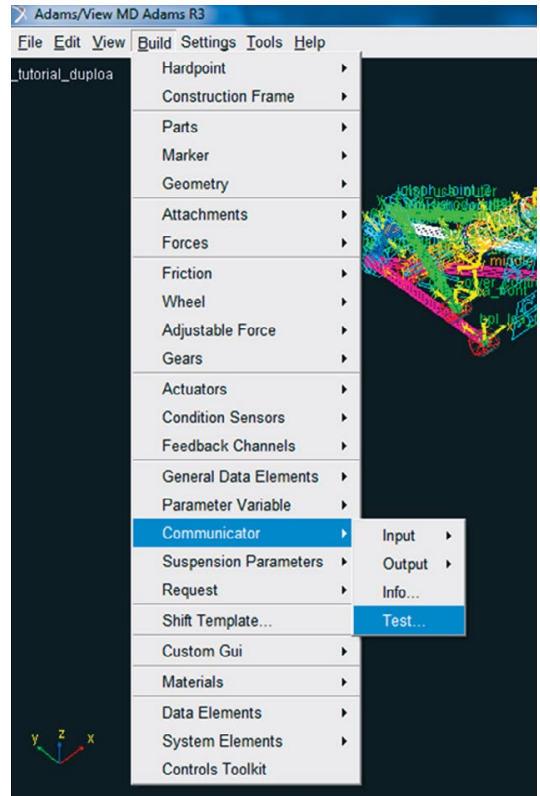

Figura 5.156 - Realizando teste de compatibilidade dos Communicators com o dispositivo de teste MDI_Suspension_Testrig.

$\mathrm{Na}$ janela, Test Communicators, verifique os subsistemas a serem testados. O campo Model Names deve ser preenchido pelo usuário. Para tanto, sobre o campo, clique com o botão direito do mouse. Nesse momento, aparecerá uma caixa de diálogo solicitando o modelo (Model). Passe o cursor por cima e selecione a opção Guesses. Os nomes dos modelos disponíveis aparecerão, e o usuário deverá selecionar primeiramente o tutorial_duploa e, em seguida, o MDI_Suspension_Testrig.

Nesse caso, observa-se na Figura 5.157 que o teste será feito entre o subsistema da suspensão (_tutorial_duploa) em construção e do subsistema correspondente a uma plataforma de teste, denominado MDI_Suspension_Testrig.

Os parâmetros contidos no campo Minor Roles "front" e "any" devem ser digitados pelo usuário.

- $\quad$ Clique em $O K$.

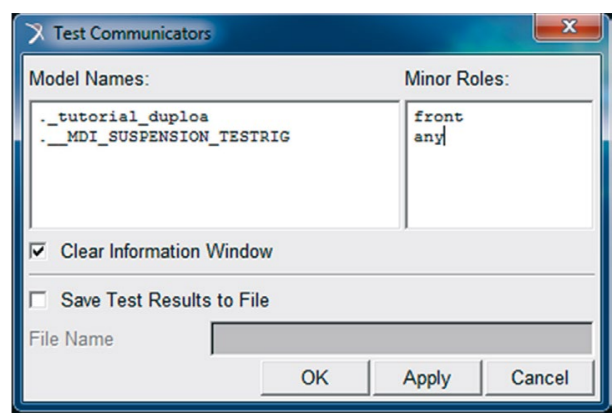

Figura 5.157 - Subsistemas a serem submetidos a teste de compatibilidade de Communicators. 
A janela mostrada na Figura 5.158, denominada Information, será aberta e, nela, é possível verificar se há algum erro de comunicação entre Communicators para que possam ser realizadas as correções necessárias.

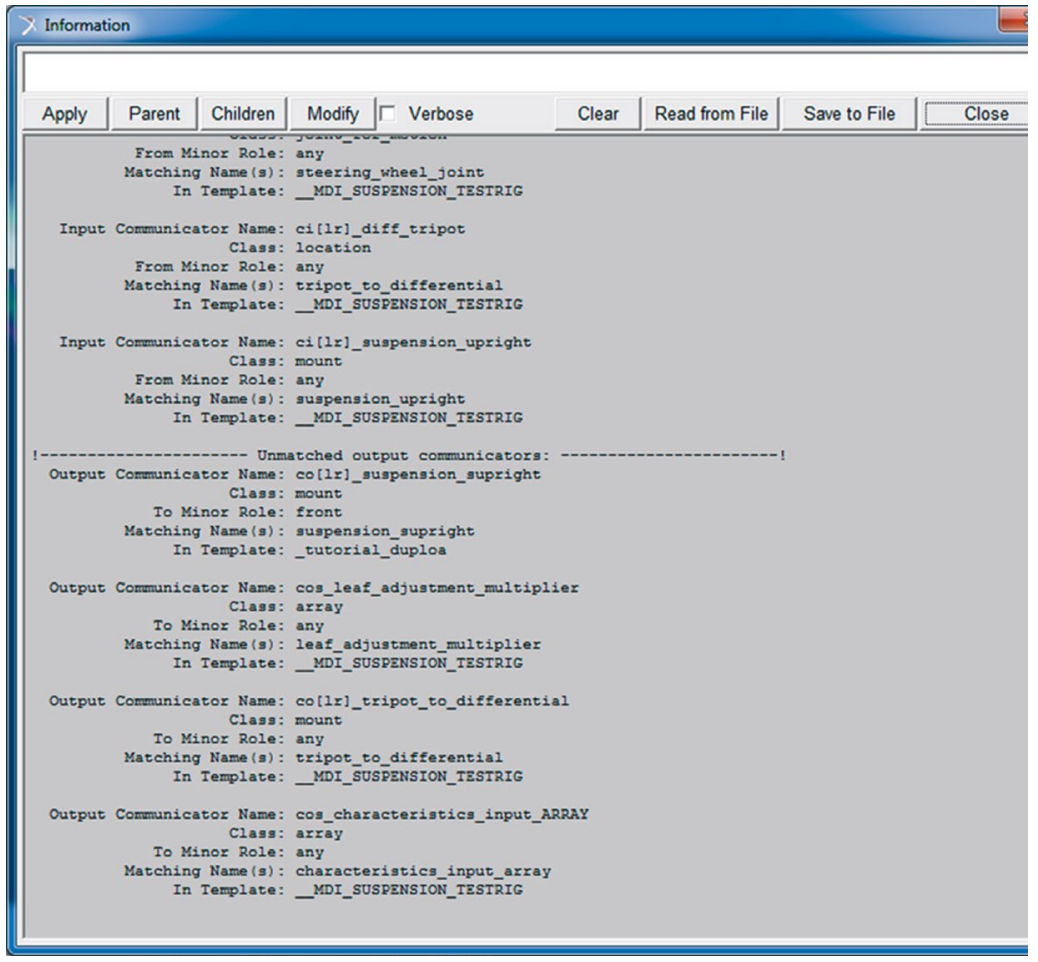

Figura 5.158 - Informação de compatibilidade dos communicators.

Note que a lista de Unmatched input communicators e Unmatched output communicators não significa propriamente erro de projeto. Mostra apenas, como é o caso, que há Communicators que não encontraram seu correspondente. De fato, isto acontece por não se estar testando todo o veículo, mas apenas a suspensão dianteira com o dispositivo de teste virtual Testrig.

O Template da suspensão está, agora, finalizado.

- Feche a janela, selecionando a opção Close, em seu canto superior direito.

- Salve seu projeto. 


\section{Etapa 24 - Verificação das juntas: aplicando o Kinematic Mode}

Um importante passo para a correta simulação da suspensão Duplo A será apresentada nesta Etapa. De fato, durante a construção do projeto, juntas e buchas foram inseridas e precisam de ajustes em sua função.

Nesse sentido, serão feitas modificações nas juntas Joint_5 e Joint_6. Lembrando que essas juntas são as revolutas e foram iseridas entre as partes gel_Lower_control_arm e o mtl_subframe_to_body; gel_Upper_control_arm e o mtl_ uca_to-body, respectivamente.

A notar: Durante a construção do projeto de uma suspensão, há duas maneiras possiveis de se estabelecer como as partes (Parts), que o compõe, irão reagir umas em relação às outras. Essas relações podem ser por meio do uso de Buchas (Bushings) ou pelo uso de Juntas (Joints).

Buchas: permitem seis graus de liberdade entre as partes (três translações e três rotações). No entanto, em sua construção, é possível especificar a rigidez, pré-carga (Preload) e ser offset.

Juntas: estabelecem uma relação cinemática entre as partes, fornecendo graus de liberdade e restringindo movimentos. As juntas, normalmente, são especificadas de acordo com a ideia de gran de liberdade e restrição de movimento que se deseja no modelo.

Na janela de criação das buchas (Bushing) Build $\rightarrow$ Attachments $\rightarrow$ Bushing $\rightarrow$ New há um campo denominado Inactive, enquanto na criação de juntas (Joints) Build $\rightarrow$ Attachments $\rightarrow$ Joint $\rightarrow$ New há outro campo denominado Active. No caso de se criar buchas e juntas no mesmo local de conexão de uma parte (Part) em um dado modelo, o usuário pode alternar a função entre as duas categorias de Attachments, da seguinte forma: ao tornar o Kinematic Mode ativo na junta (basta selecionar esta opção), selecione no campo Inactive da bucha a opção Kinematic Mode. Desta forma, apenas a junta atuará em simulações cinemáticas.

Geralmente, a junta se torna inativa em análises dinâmicas, se existe uma bucha. Isso porque a bucha é capaz de modelar tanto o movimento, quanto inserir características de rigidez e amortecimento entre as Parts. A junta tem papel definido no que tange a análise cinemática apenas.

- Desta forma, clique com o botão direito sobre a Joint_5 (Figura 5.124) e altere o campo denominado Active para a opção kinematic mode como é mostrado a seguir, Figura 5.159

- $\quad$ Em seguida, faça o mesmo com a Joint_6 (Figura 5.126), alterando o campo denominado Active para a opção kinematic mode. 


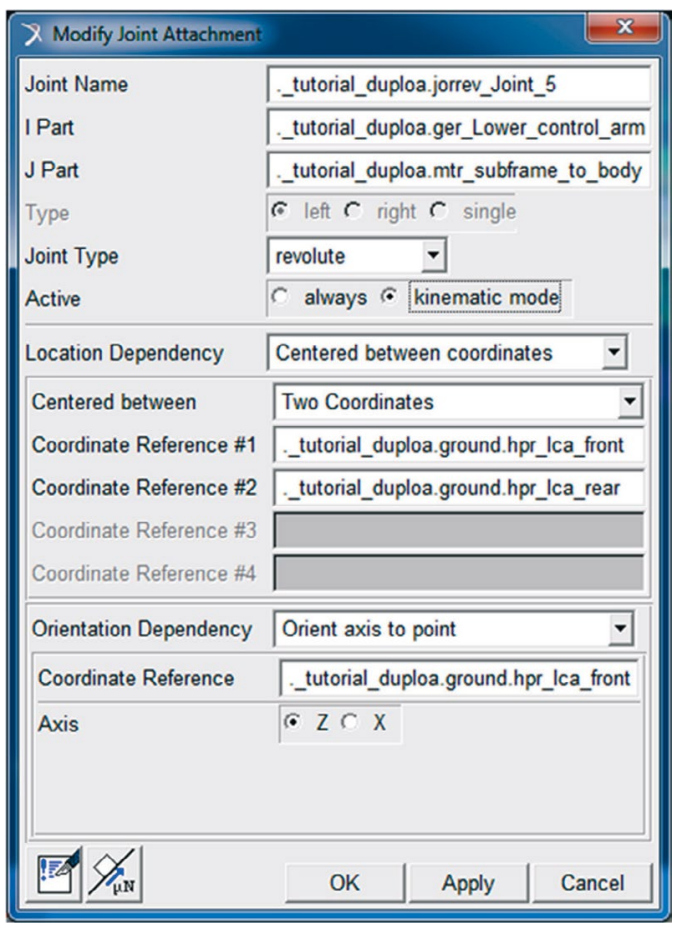

Figura 5.159 - Alterando para kinematic mode a opção Active em ambas as juntas jolrev_Joint_5 e jolrev_Joint_6.

- $\quad$ Salve seu projeto.

Etapa 25 - Criação do subsistema associado ao Template_tutorial_duploa (Subsystem)

Uma vez concluído o projeto da suspensão (Template), se faz necessária a criação do subsistema da suspensão (Subsystem) para que se possa realizar as devidas simulações no modelo criado. Neste sentido:

- $\quad$ Ainda na tela principal do Template, salve o mesmo e aperte a tecla F9 do teclado. Esta ação resulta na transferência para o ambiente Standard do ADAMS/Car destacado na Figura 5.160. Repare no canto inferior esquerdo da tela mostrada na referida figura, onde se lê Switching modes to: Standard ADAMS/Car.... 


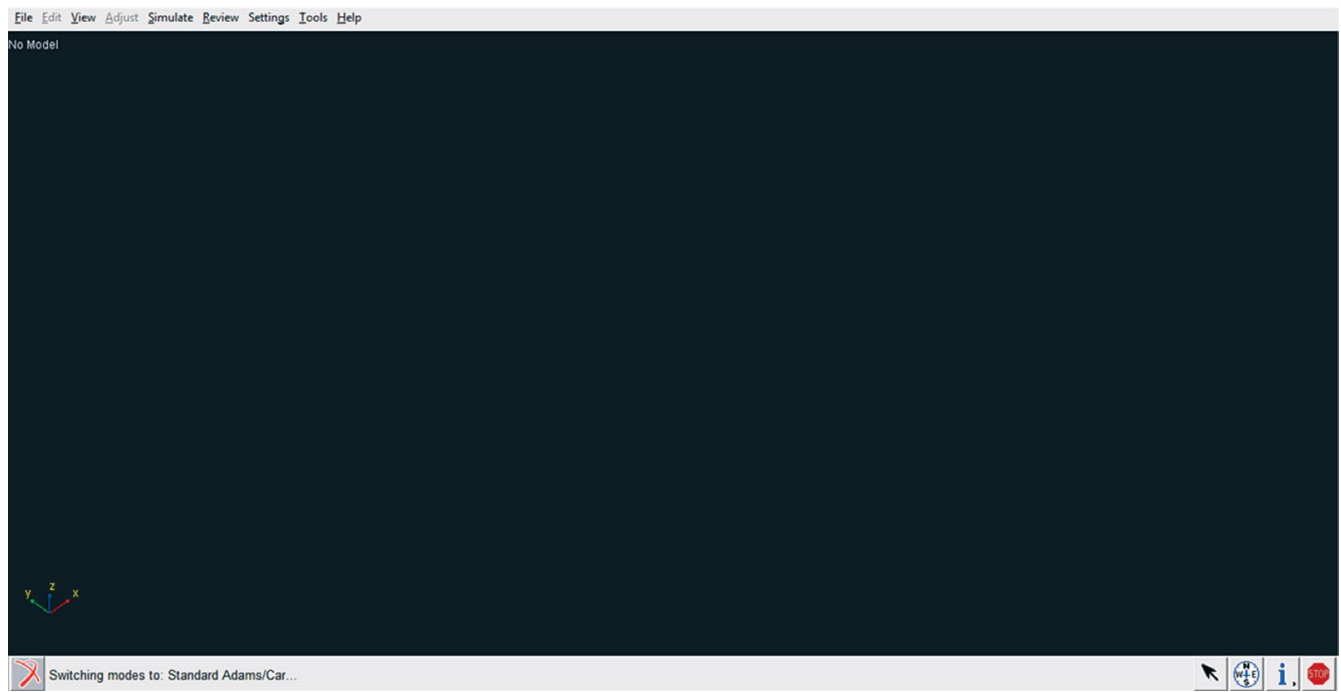

Figura 5.160 - Interface do modo Standard.

- $\quad$ Para criar o Subsystem, acesse o menu File $\rightarrow$ New $\rightarrow$ Subsystem (Figura 5.161).

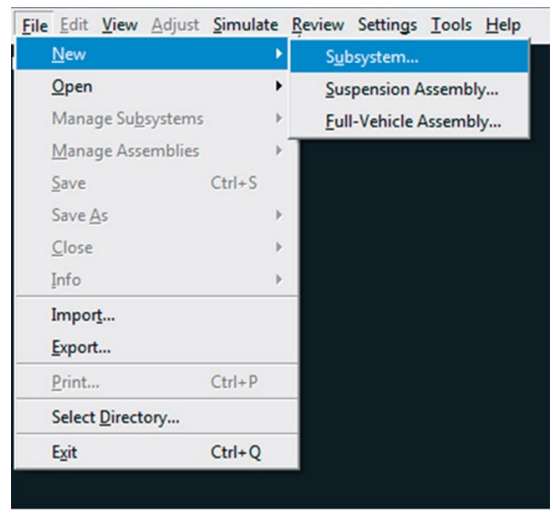

Figura 5.161 - Janela correspondente à criação de um Subsystem.

- Preencha os campos da janela New Subsystem, conforme ilustrado na Figura 5.162. Note que, como o projeto é de uma suspensão dianteira o campo Minor Role, deve estar selecionado com a opção front. O nome de Subsystem pode ser o mesmo já designado no Template.

- $\quad$ Clique em $O K$. 


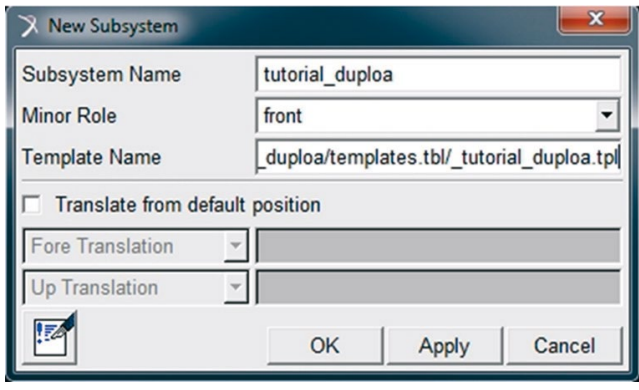

Figura 5.162 - Preenchimento da caixa de diálogo para criação de um Subsystem.

- Caso o Template esteja aberto, a janela ilustrada na Figura 5.163 será aberta. Para confirmar, clique em Yes.

Q.1. Question

Figura 5.163 - Confirmação de utilização do Template em execução.

A Figura 5.164 ilustra o Subsystem criado.

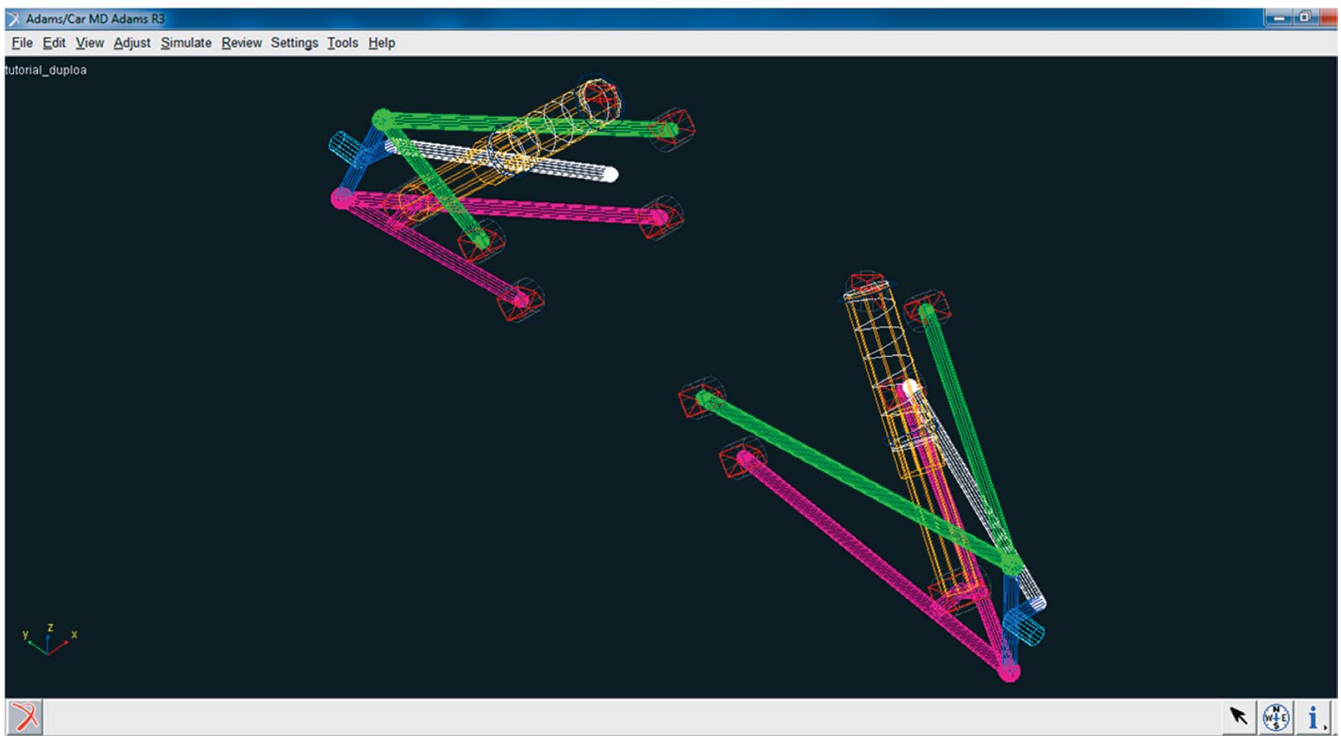

Figura 5.164 - Ilustração do subsistema gerado a partir do Template_tutorial_duploa. 
- Salve o subsistema Para tanto acesse o menu principal do ambiente Standard selecione a opção File $\rightarrow$ Save.

\section{Etapa 26 - Criação do Assembly associado ao Template_tutorial_duploa(Subsystem)}

Nesta etapa, será criado o Assembly, que configura o próximo passo após a criação do Subsystem, para que se possam realizar simulações cinemáticas e dinâmicas com o modelo criado.

O Assembly configura uma montagem entre subsistemas e no ADAMS/Car pode ser escolhido entre as seguintes opções: Suspension Assembly ou Full-Vehicle Assembly. Como, nesse caso, a simulação será realizada apenas com o subsistema suspensão e uma plataforma de testes, a primeira opção será escolhida.

- Ainda na tela onde foi criado o subsistema da suspensão, acesse o menu File $\rightarrow$ New $\rightarrow$ Suspension Assembly (Figura 5.165).

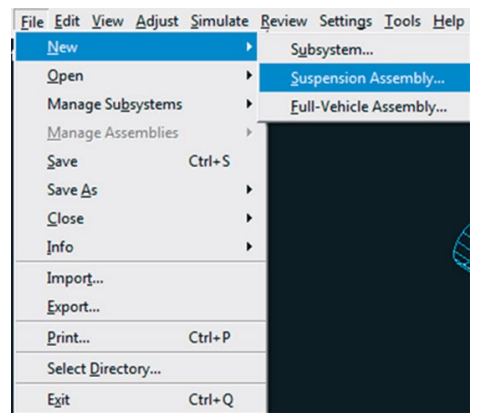

Figura 5.165 - Janela correspondente à criação de um Assembly.

A Figura 5.166 ilustra o preenchimento da janela New Suspension Assembly.

- Atribua um nome ao Assembly no primeiro campo; este já deve ser diferente do nome atribuído ao Subsystem.

- Selecione o subsistema de interesse (segundo campo). No campo Suspension Test Rig a opção_MDI_SUSPENSION_TESTRIG deve estar selecionada, a qual corresponde à plataforma de teste a ser utilizada nas simulações. Caso deseje incluir outros subsistemas na simulação, selecione o item Other Subsystems.

- $\quad$ Clique em $O K$. 


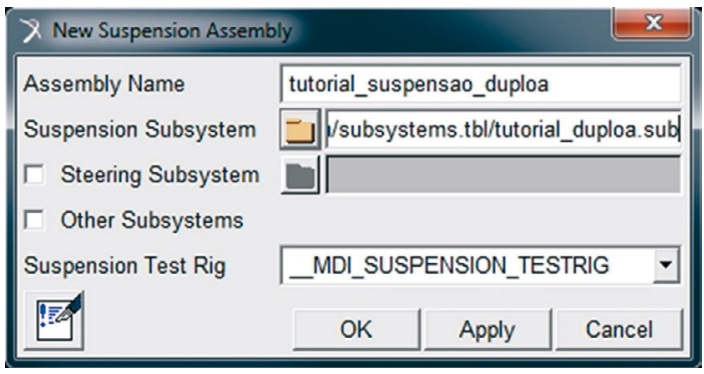

Figura 5.166 - Criação do Assembly.

Caso o Template do projeto esteja aberto, aparecerá a mensagem mostrada na Figura 5.167. Nesse caso, clique sobre a opção Yes.

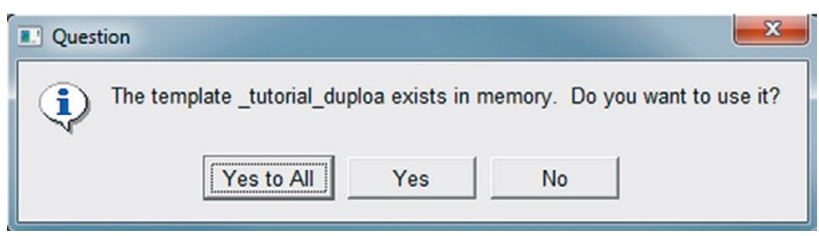

Figura 5.167 - Confirmação de utilização do Template em execução.

A Figura 5.168 ilustra a tela de confirmação de criação do Assembly "Message Window”. Esta mesma janela poderá fornecer a informação de algum erro ocorrido durante a criação do Assembly.

- Após verificação de mensagens, clique em Close.

- Salve seu projeto.

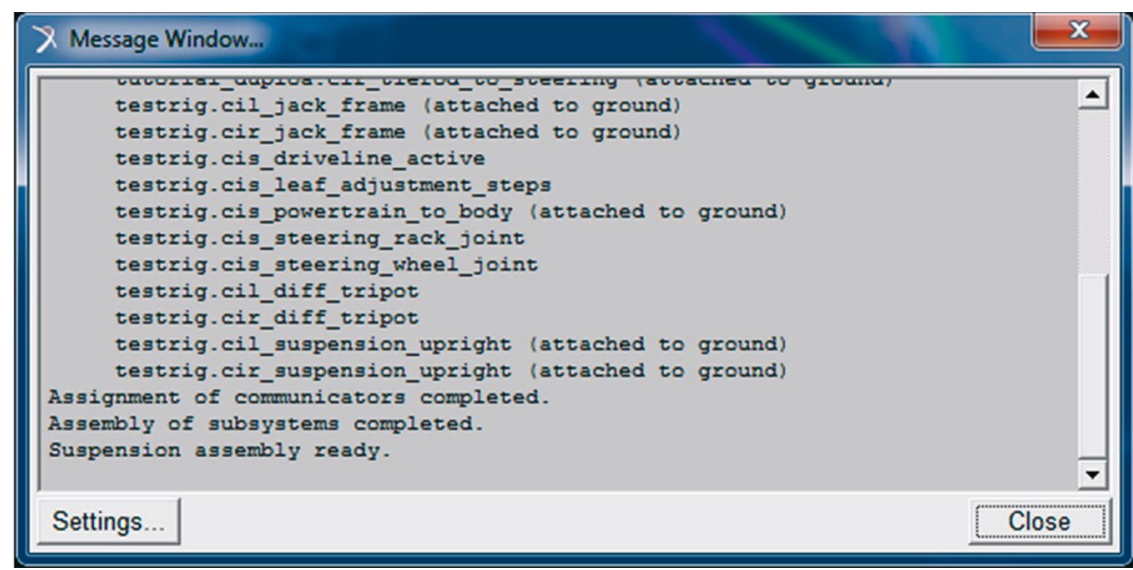

Figura 5.168 - Tela de confirmação de criação do Assembly. 
A Figura 5.169 ilustra o Assembly construído.

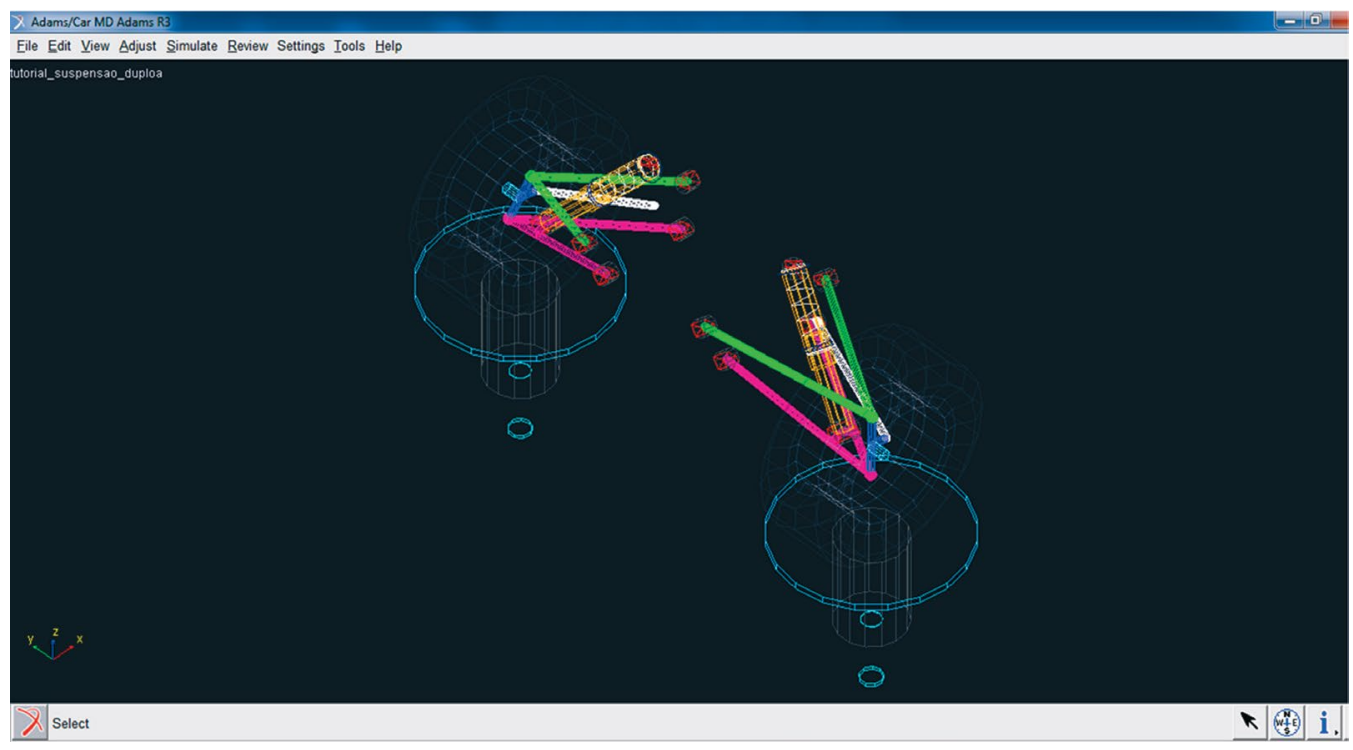

Figura 5.169 - Ilustração do Assembly construído.

\section{Etapa 27 - Simulação do Assembly_tutorial_suspensao_duploa e_MDI_ SUSPENSION_TESTRIG}

Nesta etapa, será realizada a simulação do Assembly, criado na Etapa 26.

Primeiramente, defina o diretório para armazenar os resultados gerados na simulação. Para tanto:

- Acesse o menu File $\rightarrow$ Select directory (Figura 5.170).

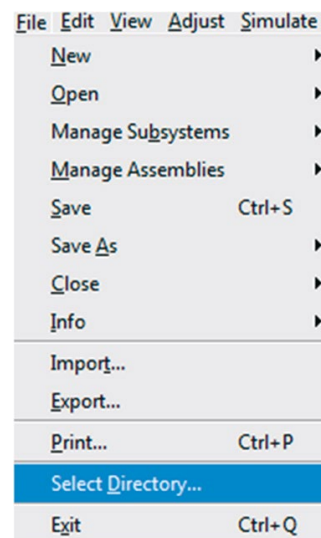

Figura 5.170 - Janela correspondente à seleção do diretório para simulações. 
- $\quad$ Selecione o diretório de interesse (Figura 5.171).

- $\quad$ Clique em $O K$.

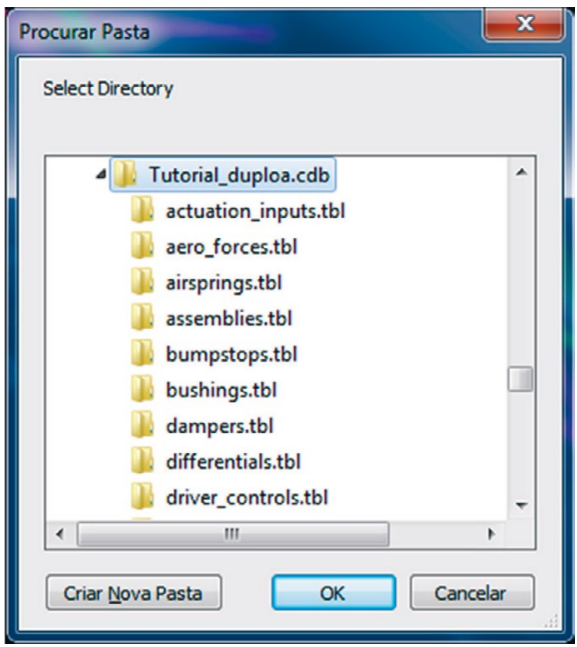

Figura 5.171 - Janela correspondente à seleção de diretório para gravação de simulações

A simulação proposta aqui corresponde a uma solicitação vertical e paralela na suspensão (left e right). Essa simulação permite analisar as características geométricas do subsistema, durante todo seu curso vertical. Para isto:

- Acesse o menu Simulate $\rightarrow$ Suspension Analysis $\rightarrow$ Parallel Wheel Travel (Figura 5.172).

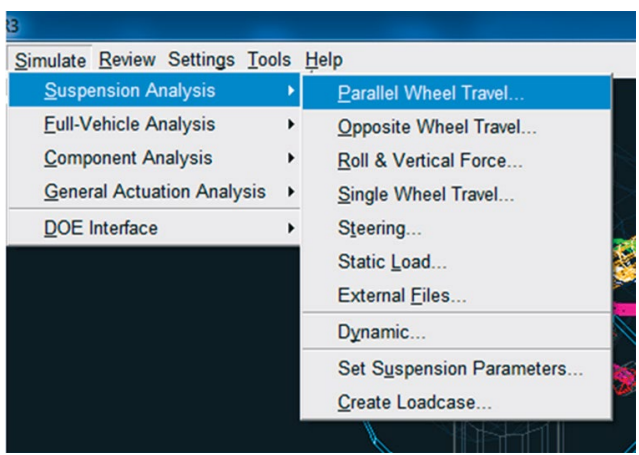

Figura 5.172 - Selecionando o tipo de simulação.

Os parâmetros de simulação devem ser definidos na janela Suspension Analysis: Parallel Travel, conforme a Figura 5.173. Note que, na simulação proposta, o percurso total corresponde a $100 \mathrm{~mm}$ ( $50 \mathrm{~mm}$ no sentido positivo, ou Bump e 50 mm no sentido negativo, Rebound).

- Ao finalizar o preenchimento, clique em $O K$. 


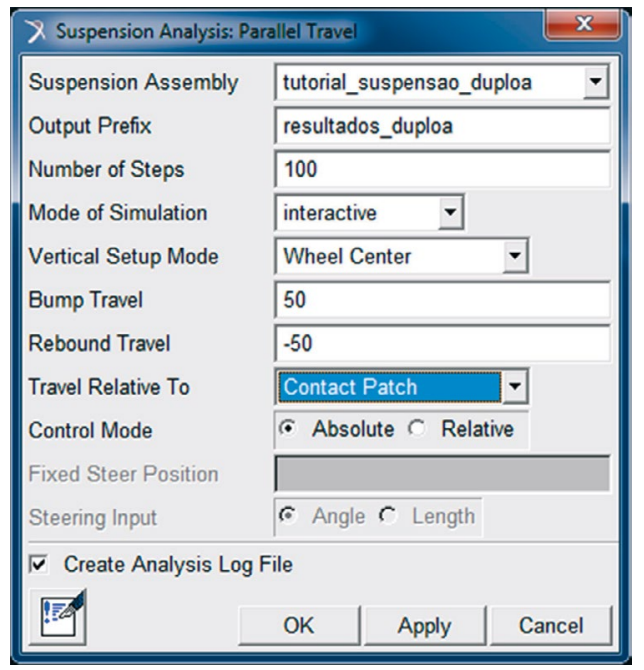

Figura 5.173 - Janela correspondente à definição dos parâmetros de simulação.

A Figura 5.174 ilustra que a simulação foi executada.

- Clique em Close.

- $\quad$ Salve seu projeto.

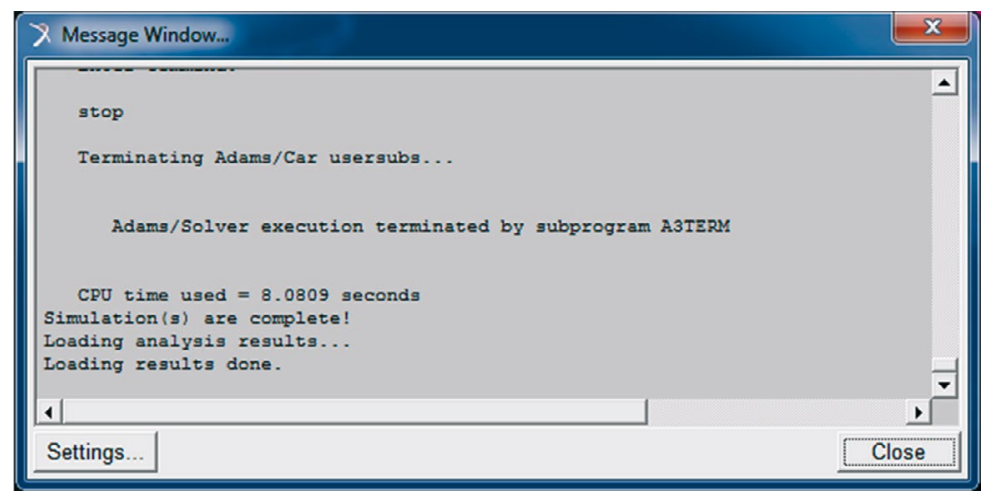

Figura 5.174 - Caixa de diálogo informando que a simulação foi executada.

\section{Etapa 28 - Visualização da simulação do Assembly: animação do modelo virtual para obtenção de resultados}

Nesta etapa, o modelo da suspensão criado será visualizado, mediante animação do carregamento vertical aplicado, conforme ensaio virtual estabelecido pela opção Paralell Wheel Travel.

Para melhor visualização do modelo da suspensão durante a animação, o usuário pode optar pelo modo Shaded. Para isto: 
- Clique com o botão direito do mouse na área de trabalho principal (em qualquer ponto que não seja sobre o modelo).

- Selecione a opção Shaded $\langle S>$ (Figura 5.175).

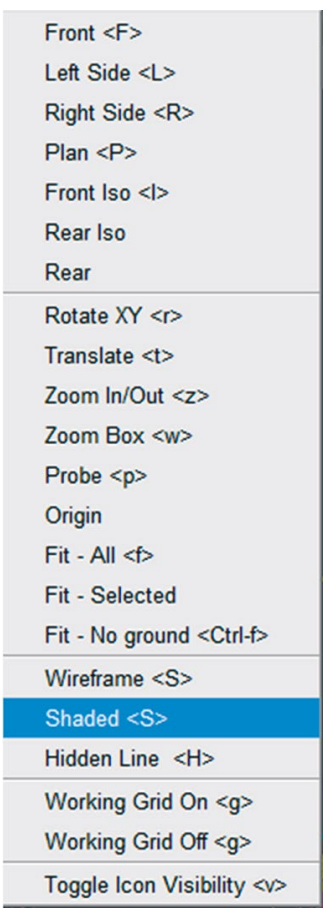

Figura 5.175 - Janela correspondente à seleção do modo de visualização Shaded.

Em seguida:

- $\quad$ Acesse o menu Review $\rightarrow$ Animation Controls (Figura 5.176).

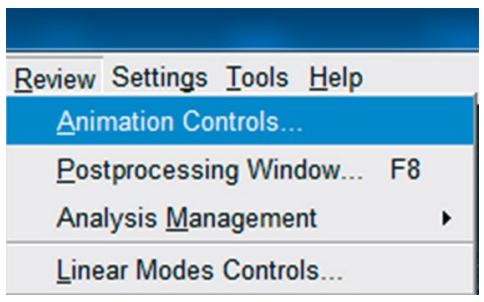

Figura 5.176 - Janela correspondente à animação do modelo da suspensão.

- Na janela Animation Controls preencha os campos, conforme a Figura 5.177. O usuário pode definir os parâmetros de animação, de acordo com sua preferência. Segue como sugestão os valores especificados na Figura 5.177. 
Logo abaixo das teclas de controle de animação, a barra que oscila entre $I n c$ e +Inc, permite ao usuário, dentro de um ciclo de simulação (Cycles), avançar ou recuar, a fim de escolher um ponto de análise (Frame). O campo Time Range dá ao usuário a possibilidade de definir um intervalo de tempo para realização da simulação. A aba Cycles permite escolher quantas vezes a simulação irá se repetir, no caso, cinco vezes. O campo Frame Increment permite ao usuário definir de quantos em quantos Frames ele deseja saltar, no caso, o incremento será de um em um.

- Para inicializar a animação, clique em $\triangleright$.

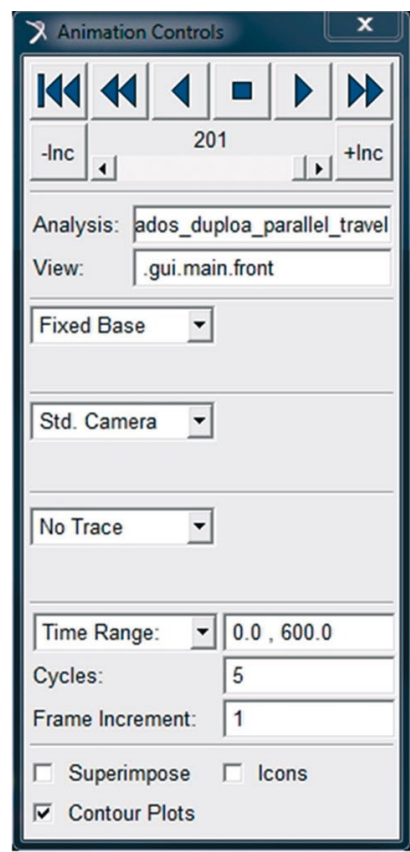

Figura 5.177 - Definição dos parâmetros de animação.

A Figura 5.178 ilustra como o modelo será apresentado na tela principal. A animação pode ser recomeçada selecionando o item $\bullet$. 


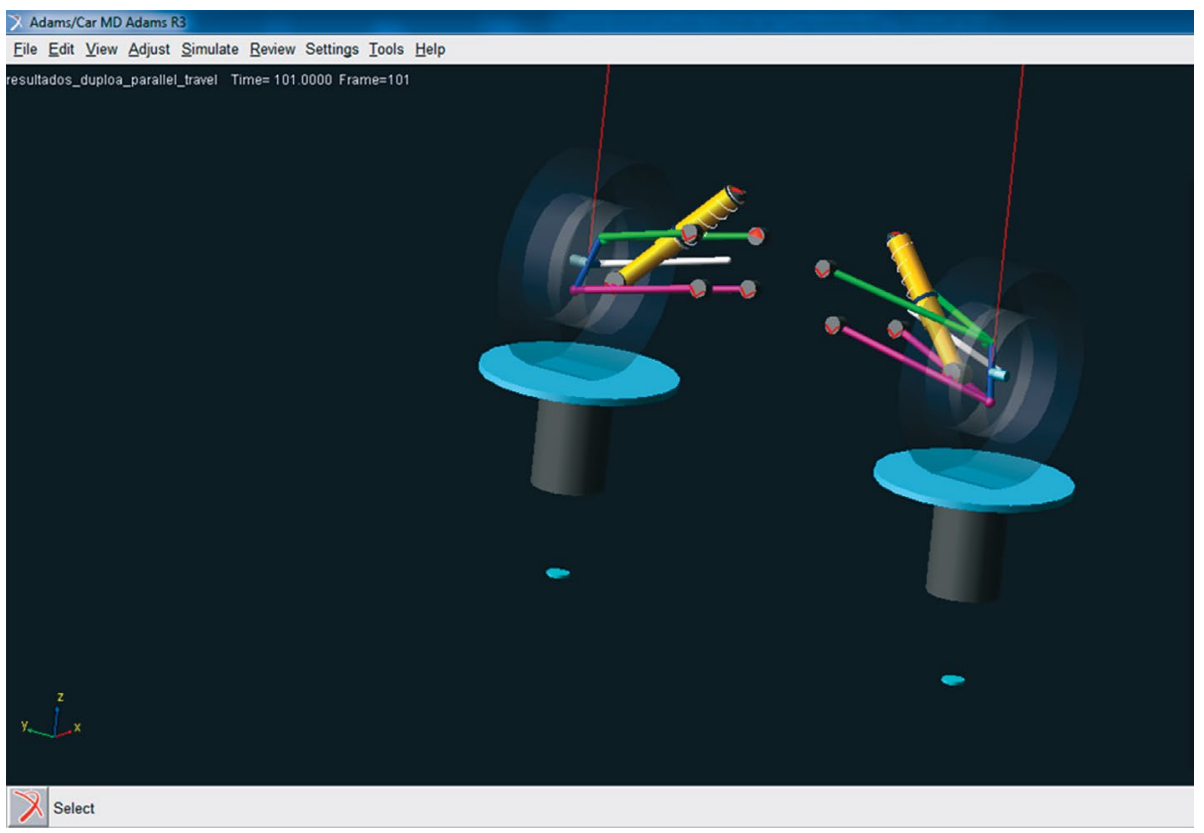

Figura 5.178 - Ilustração da tela referente à animação do Assembly.

\section{Etapa 29 - Obtenção de resultados: ADAMS/Car Postprocessing}

Nesta etapa, os resultados serão gerados em forma de gráficos, facilitando, assim, sua análise. Para realizar essa análise, proceda como sugerido a seguir:

- Acesse o menu Review $\rightarrow$ Postprocessing Window. Caso deseje, o usuário poderá utilizar o atalho correpondente à tecla F8 do teclado. A Figura 5.179 ilustra a interface PostProcessing. 


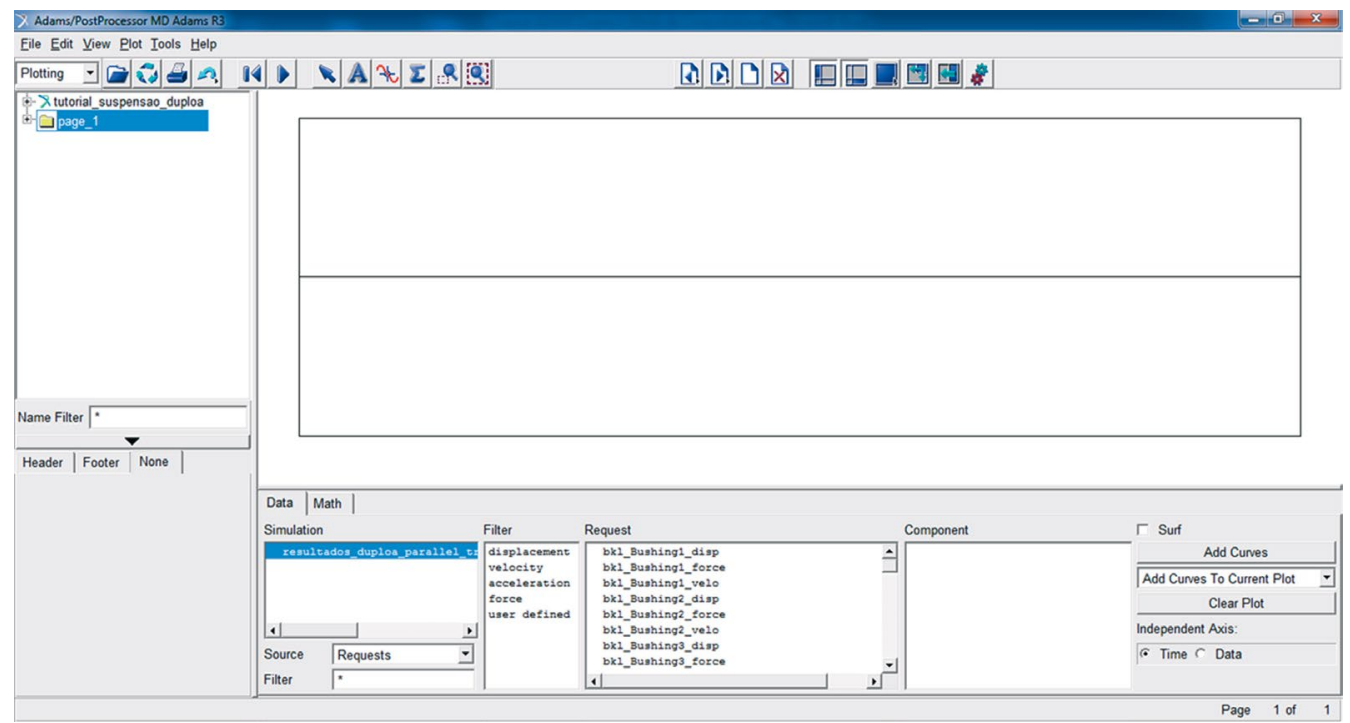

Figura 5.179 - Interface PostProcessing.

- Para criar os gráficos, acesse o menu Plot $\rightarrow$ Create Plot (Figura 5.180).

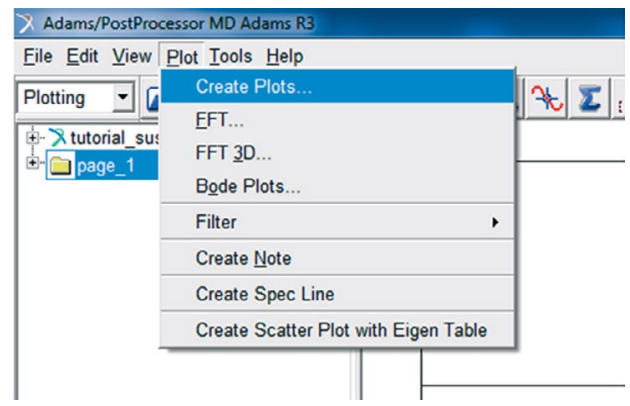

Figura 5.180 - Janela correspondente à criação de gráficos.

A janela File Import será aberta.

- Selecione o tipo de arquivo: Plot Configuration (*.plt).

- $\mathrm{O}$ arquivo com a configuração desejada dos resultados deve ser selecionado no campo Plot Configuration File. Para isto, clique com o botão direito do mouse sobre esse campo, e selecione a opção mostrada na Figura 5.181. 


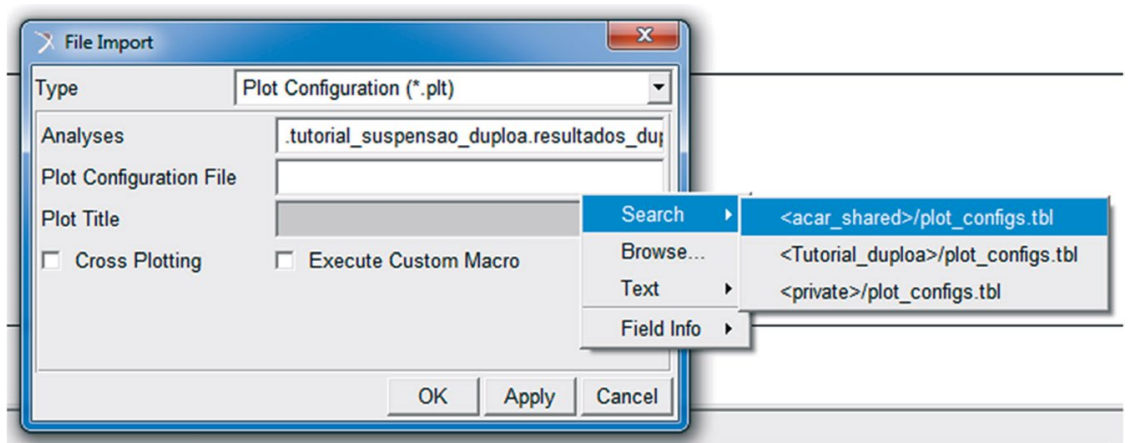

Figura 5.181 - Janela correspondente à importação de arquivos da biblioteca de configuração de gráficos do ADAMS/Car.

- Na biblioteca do ADAMS/Car, já existem diversas opções disponíveis de configurações de gráficos. Nesse caso, como se está interessado nos resultados do Assembly relacionados à Suspensão, selecione a opção mdi_suspension_short.plt (Figura 5.182).

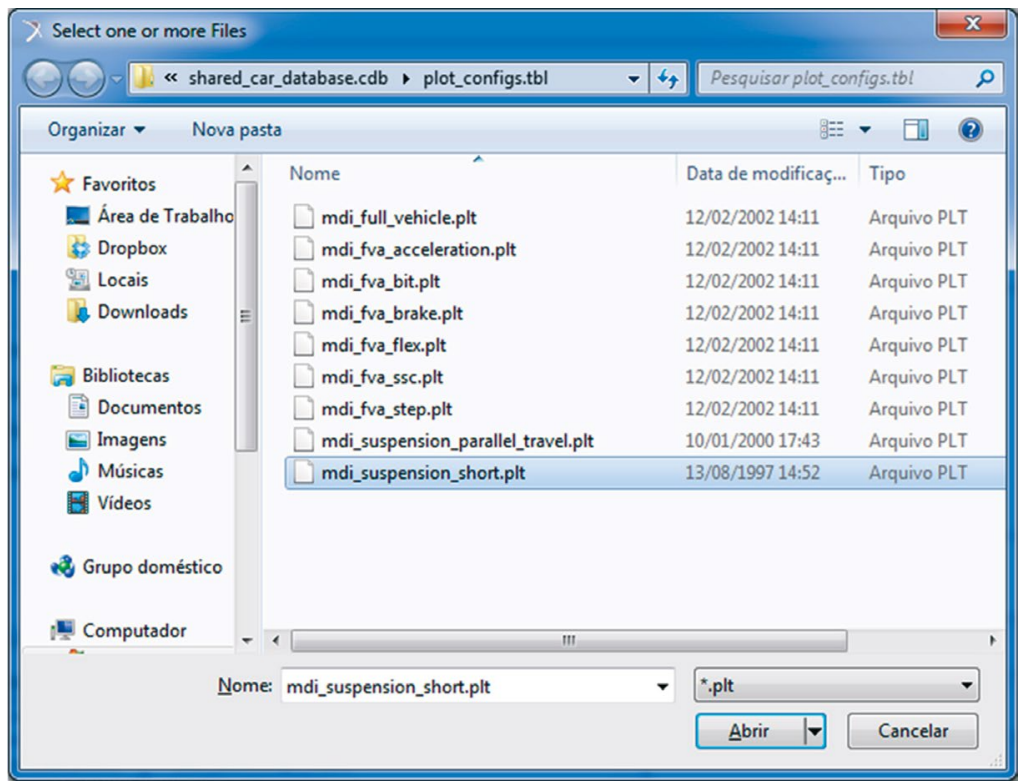

Figura 5.182 - Janela correspondente à definição de arquivo de configuração de gráficos.

$\mathrm{O}$ arquivo mdi_suspension_short.plt gera automaticamente nove gráficos, sendo eles: anti dive, camber angle, caster moment arm, roll camber coefficient, roll center height, roll steer, scrub radius, toe angle e wheel rate. 
A Figura 5.183 ilustra a janela File Import, após preenchimento dos campos necessários.

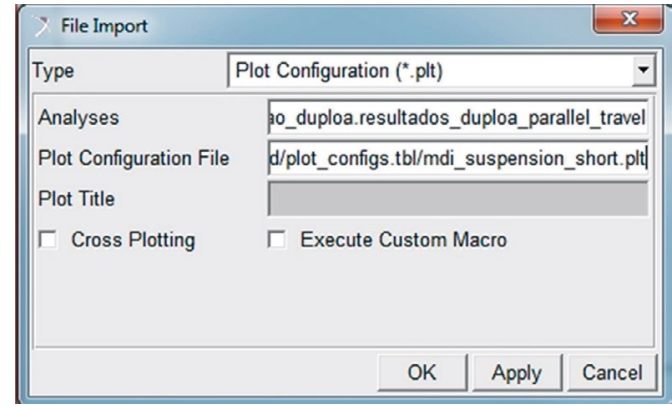

Figura 5.183 - Janela correspondente à importação de arquivos para obtenção de gráficos no Postprocessor.

- $\quad$ Clique em $O K$.

- Para alternar entre os gráficos obtidos, selecione os ícones [a]

Como exemplo, o sétimo gráfico gerado corresponde ao parâmetro Caster Moment Arm (eixo vertical) em função do curso da roda (Wheel Travel), como pode ser observado na Figura 5.184.

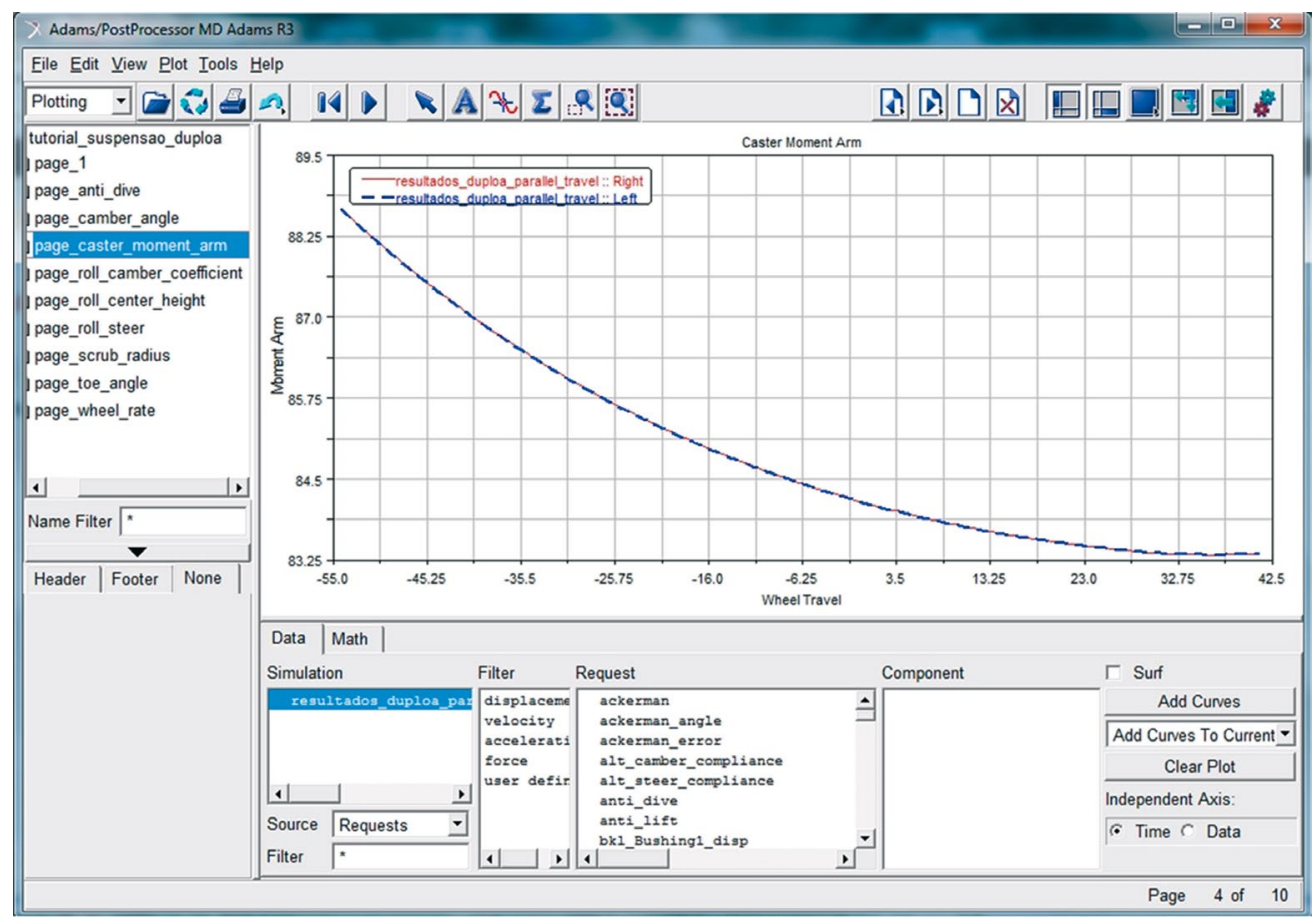

Figura 5.184 - Gráfico Caster Moment Arm x Wheel Travel. 
A notar: O termo Wheel Travel define o movimento das rodas do veículo, no caso, as rodas do eixo dianteiro, provocado pelo trabalho vertical da suspensão (ride) e, também, o movimento destas, ocasionado pelo uso do sistema de direção.

O Caster representa uma das medidas de geometria da suspensão, podendo ser definido em vista lateral do veículo, como o ângulo que o eixo de direção faz com uma linha vertical. Essa linha vertical representa um referencial, que passa pelo centro da roda. O eixo da direção, por sua vez, no presente modelo virtual, passará pelas juntas situadas nos Hardpoints hpl_uca_outer $e$ hpl_lca_outer. Perceba, pela construção do modelo, aqui proposto que essa reta conteráo hpl_tierod_outer.

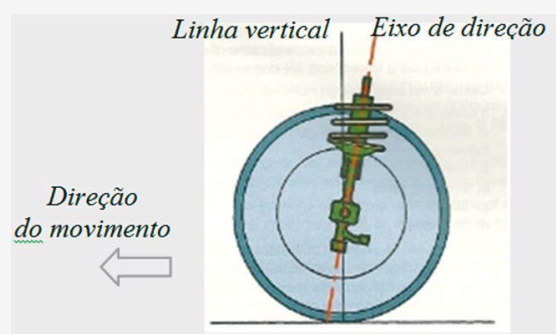

(a)

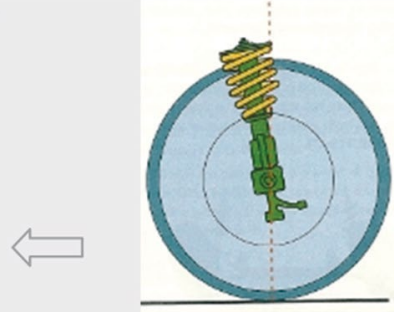

(b)

Figura representativa do Caster: (a) Caster positivo; (b) Caster negativo.

Fonte: HALDERMAN J. D. Automotive technology: principles, diagnosis and service, New Jersey, Pearson, 2012.

Seu valor é positivo quando o pivô superior, junta superior, está atrás do pivô inferior. No caso negativo observa-se o contrário.

Considerando o caso de um Caster positivo, o Caster Moment Arm representa um braço de alavanca que é medido a partir do centro da roda até o ponto em que o eixo de direção toca a linha horizontal que passa por esse centro. De acordo com a figura

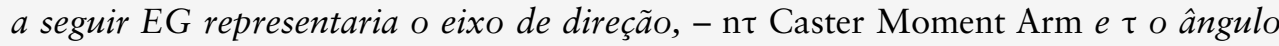
de Caster.

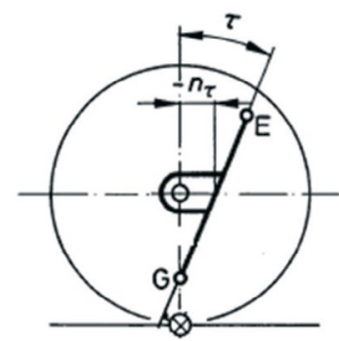

Figura representativa do Caster Moment Arm.

Fonte: REIMPELL, J.; STOLL, H.; BETZLER, J. W. The automotive chassi: engineering principles, Butterworth-Heinemann, 2001. 
- Caso deseje apagar algum gráfico(s), selecione-o na árvore de diretórios - Treeview (à esquerda da tela), conforme ilustrado na Figura 5.185. Recomenda-se apagar individualmente cada gráfico. $\mathrm{O}$ atalho $\mathrm{Ctrl}+\mathrm{X}$ também pode ser usado para executar a ação de excluir o gráfico.

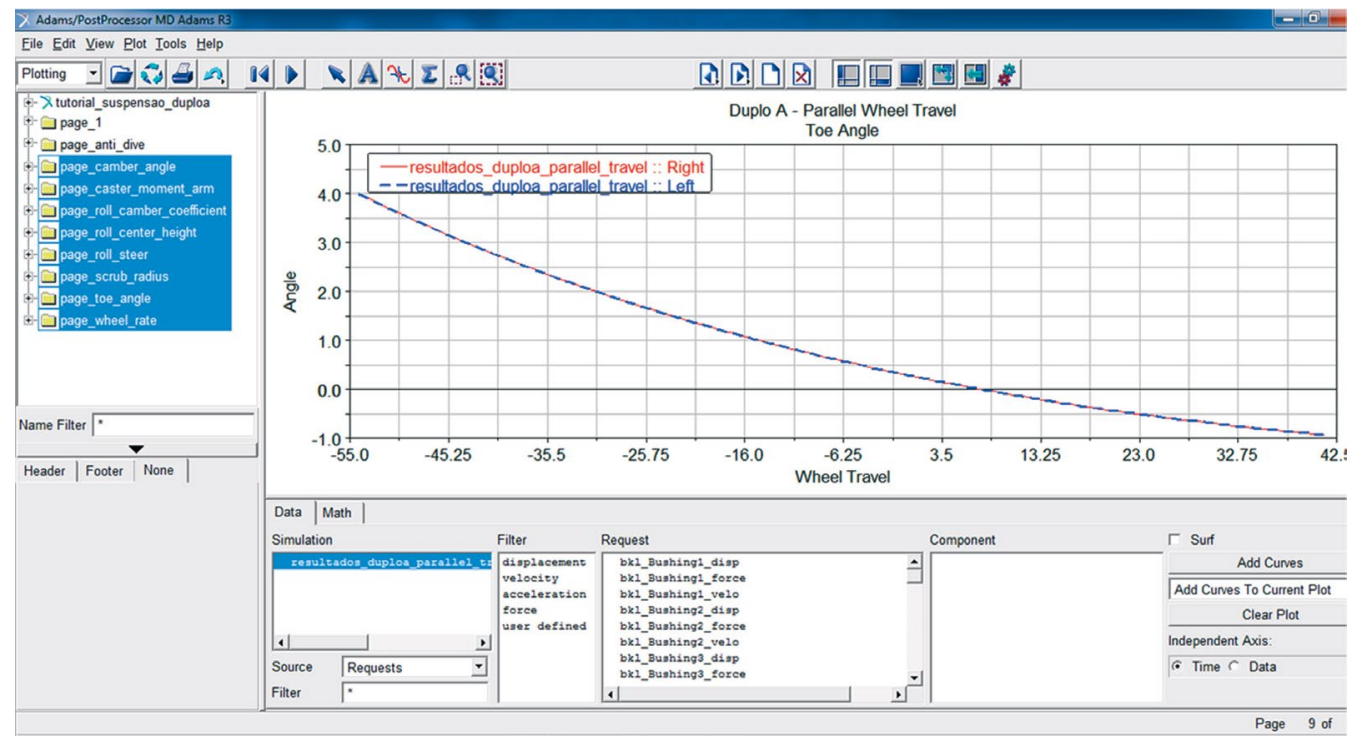

Figura 5.185 - Selecionando gráficos para serem apagados.

- $\quad$ Acesse o menu Edit $\rightarrow$ Delete (Figura 5.186).

\begin{tabular}{|c|c|}
\hline dams/PostProcessor & MD Adams R: \\
\hline Edit View Plot & Iools ㅂelp \\
\hline Undo & Ctrl $+Z$ \\
\hline Redo & Ctrl + Shift $+Z$ \\
\hline Copy & $\mathrm{Ctrl}+\mathrm{C}$ \\
\hline Paste & Ctrl + V \\
\hline Rename... & \\
\hline Delete & Ctrl+X \\
\hline Preferences. & \\
\hline Deselect All & Ctrl $+D$ \\
\hline
\end{tabular}

Figura 5.186 - Excluindo gráficos.

- Para sair da interface PostProcessing, pressione a tecla F8 ou acesse o menu File Close Plot Window. 


\section{Etapa 30 - Salvando as configurações gráficas}

Para salvar as modificações nos gráficos gerados anteriormente o usuário deve seguir os passos descritos nesta etapa.

- $\quad$ No menu File, selecione Export e, em seguida, selecione Plot Configuration File (Figura 5.187).

\begin{tabular}{|c|c|c|c|c|}
\hline \multicolumn{5}{|c|}{ Eile Edit View Plot Iools $\underline{H}$ elp } \\
\hline \multicolumn{2}{|c|}{ Replace Simulations... } & DA & \&|A & $\frac{7}{t}$ \\
\hline \multicolumn{2}{|l|}{ Import } & & & \\
\hline \multicolumn{2}{|l|}{ Export } & \multicolumn{3}{|c|}{ Plot Configuration File. } \\
\hline Print... & Ctrl $+\mathrm{P}$ & \multicolumn{3}{|c|}{ Numeric Data... } \\
\hline \multicolumn{2}{|c|}{ Select Directory... } & \multicolumn{3}{|c|}{ Sppreadsheet... } \\
\hline Close Plot Window & F8 & \multicolumn{3}{|c|}{ Iable... } \\
\hline \multirow{6}{*}{$\begin{array}{l}\text { maxis } \\
\text { maxis }\end{array}$} & & \multirow{2}{*}{\multicolumn{3}{|c|}{$\begin{array}{l}\text { DAC File... } \\
\text { RPC File... } \\
\text { ㅂTML Report... }\end{array}$}} \\
\hline & & & & \\
\hline & & \multirow{4}{*}{\multicolumn{3}{|c|}{$\begin{array}{l}\text { Graphics File... } \\
\text { Request File... } \\
\text { Results File... } \\
\text { Analysis Files... }\end{array}$}} \\
\hline & & & & \\
\hline & & & & \\
\hline & & & & \\
\hline
\end{tabular}

Figura 5.187 - Janela correspondente à exportação de arquivos gráficos.

A janela Save Plot Configuration File será aberta. No campo Configuration File Name, digite Parameters_duploa. Selecione a opção All Plots (Figura 5.188).

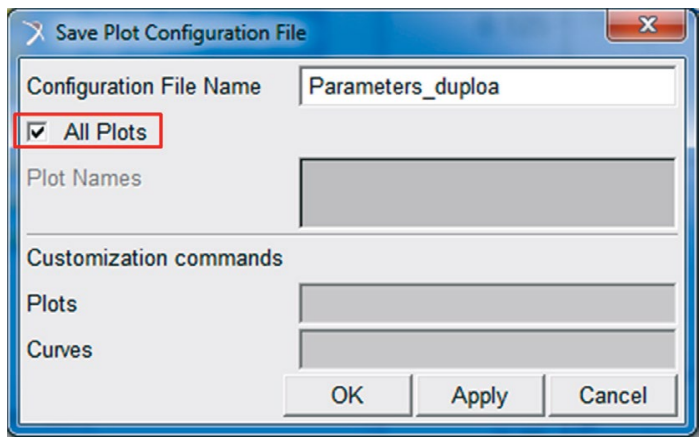

Figura 5.188 - Janela correspondente à definição do arquivo de exportação.

- $\quad$ Clique em $O K$.

- $\quad$ No menu File, selecione Close Plot Window ou pressione F8 para que o ADAMS/Car retorne para a janela principal.

Assim, considera-se que o presente tutorial atende o que o usuário espera em termos de construção do subsistema, no entanto, a análise dos gráficos gerados da construção fogem ao escopo da presente obra. 